\title{
Fonctions d'échelle interpolantes, polynômes de Bernstein et ondelettes non stationnaires
}

Pierre Gilles Lemarié-Rieusset

Résumé. La théorie de la convergence des fonctions d'échelle (nonstationnaires) et l'approximation des filtres d'échelle interpolants à l'aide de polynômes de Bernstein, permettent la construction d'une fonction d'échelle interpolante non-stationnaire aux propriétés d'approximation remarquables.

Abstract. The theory of convergence for (non-stationary) scaling functions and the approximation of interpolating scaling filters by means of Bernstein polynomials, allow us to construct a non-stationary interpolating scaling function with interesting approximation properties.

\section{Introduction.}

Dans cet article, nous nous intéressons essentiellement à des distributions $\varphi$ du type suivant: leur transformée de Fourier $\hat{\varphi}$, définie formellement par

$$
\hat{\varphi}(\xi)=\left\langle\varphi, e^{i x \xi}\right\rangle=\int_{-\infty}^{+\infty} \varphi(x) e^{-i x \xi} d x
$$


est un produit infini

$$
\hat{\varphi}(\xi)=\prod_{j=1}^{\infty} m_{j}\left(\frac{\xi}{2^{j}}\right),
$$

où les fonctions $m_{j}$ vérifient pour deux constantes $C_{0} \geq 1$ et $N \geq 0$ indépendantes de $j$

$$
\begin{aligned}
& m_{j} \in C^{\infty}(\mathbb{R} / 2 \pi \mathbb{Z}), \quad m_{j}(0)=1, \\
& \left\|m_{j}\right\|_{\infty} \leq C_{0}, \quad\left\|\frac{d}{d \xi} m_{j}\right\|_{\infty} \leq C_{0} j^{N} .
\end{aligned}
$$

Sous les hypothèses (3), il est facile de voir que le produit infini

$$
\prod_{j=1}^{\infty} m_{j}\left(\frac{\xi}{2^{j}}\right)
$$

converge ponctuellement, uniformément sur tout compact et dans $\mathcal{S}^{\prime}(\mathbb{R})$ vers une fonction continue $\hat{\varphi}$ à croissance lente. Pour le vérifier, il suffit d'écrire $j^{N} \leq C_{N} 2^{j / 2}$ et donc, pour $|\xi| \geq \sqrt{2}$,

$$
\begin{aligned}
|\hat{\varphi}(\xi)| & \leq \prod_{j=1}^{\infty} \min \left\{C_{0}, 1+C_{0} C_{N}\left(\frac{|\xi|}{\sqrt{2}}\right)^{j}\right\} \\
& \leq|\xi|^{2 \log C_{0} / \log 2} \prod_{j=0}^{+\infty}\left(1+C_{0} C_{N} 2^{-j / 2}\right) .
\end{aligned}
$$

En fait, il suffit de supposer que

$$
\sum_{0}^{\infty} 2^{-j}\left\|\frac{d}{d \xi} m_{j}\right\|_{\infty}<+\infty,
$$

puisque pour $|\xi| / 2^{j}$ assez petit

$$
\left|\log \left(\frac{m_{j}}{\xi / 2^{j}}\right)\right| \leq C 2^{-j}\left\|\frac{d}{d \xi} m_{j}\right\|_{\infty}|\xi| .
$$

Les produits infinis du type (2) avec $m_{j}=m_{0}$ indépendants de $j$ font depuis 1986 l'objet d'une étude intensive. Nous sommes alors dans le cadre de l'analyse multi-résolution de S. Mallat et Y. Meyer [20], [21], du 
moins lorsque $\varphi$ est de carré intégrable et que la famille $\{\varphi(x-k)\}_{k \in \mathbb{Z}}$ est une base de Riesz d'un sous-espace fermé de $L^{2}(\mathbb{R})$. Les propriétés de la fonction d'échelle $\varphi$ en fonction du filtre d'échelle $m_{0}$ ont été abondamment décrites, en particulier celles qui décrivent la régularité de $\varphi$ (A. Cohen [4], A. Cohen et I. Daubechies [6], I. Daubechies et J. Lagarias [9], T. Eirola [12], L. Hervé [14], O. Rioul [23], L. Villemoes [30], par exemple ...). Ces résultats forment ce que nous appellerons dans la suite la "théorie classique" des ondelettes, dont nous rappellerons dans la Section 2 quelques traits fondamentaux sur lesquels nous baserons la suite de nos résultats. Le résultat principal (Proposition 2) indique, sous l'hypothèse que le filtre $m_{0}$ vérifie le critère d'Albert Cohen, l'existence d'un indice $\left.\left.\sigma_{0} \in\right]-\infty,+\infty\right]$ tel que pour $s>\sigma_{0}, \varphi \notin H^{s}$ (espace de Sobolev) et pour $s<\sigma_{0}, \varphi$ est à décroissance rapide dans $H^{s}$ (i.e. pour tout $\left.k \in \mathbb{N}, x^{k} \varphi \in H^{s}\right)$; ce résultat donne de plus le calcul de $\sigma_{0}$ en fonction des propriétés spectrales de l'opérateur de transition $T_{2}$ associé à $m_{0}$, et défini par

$$
T_{2} f(\xi)=\left|m_{0}\left(\frac{\xi}{2}\right)\right|^{2} f\left(\frac{\xi}{2}\right)+\left|m_{0}\left(\frac{\xi}{2}+\pi\right)\right|^{2} f\left(\frac{\xi}{2}+\pi\right)
$$

Une première série de résultats, présentés dans la Section 3 , concerne le problème de l'approximation de la fonction d'échelle $\varphi$ par des fonctions d'échelle plus simples $\varphi_{N}$ (essentiellement, on demandera à $\varphi_{N}$ d'être à support compact). La question étudiée est essentiellement la suivante: comment l'approximation du filtre $m_{0}$ par des filtres $m_{N}$ se traduit-elle sur la qualité de l'approximation de $\varphi$ par les fonctions $\varphi_{N}$ associées? Par rapport à la "théorie classique", il s'agit donc essentiellement d'un théorème de dépendance continue par rapport aux paramètres, et il y aura donc besoin de peu d'innovation réelle pour obtenir ces résultats. (La Section 3 contient donc des résultats originaux, dont la démonstration est très courte et renvoie à la Section 2; la Section 2 contient une présentation de résultats classiques refondus pour être immédiatement opérationnels dans les sections 3 et 7).

Le design de filtres d'échelle nous imposera parfois d'introduire des filtres $m_{N}$ dont la limite n'est pas $C^{\infty}$ (par exemple, si $m_{N}$ est le $N$-ième filtre de Daubechies [8], le filtre $\left|m_{N}(\xi)\right|^{2}$ converge vers la fonction $2 \pi$-périodique $m_{\infty}$ valant 1 sur $]-\pi / 2, \pi / 2[, 1 / 2$ en $\pm \pi / 2$ et 0 sur $] \pi / 2, \pi]$; le problème de $m_{N}$ lui-même se complique encore de l'étude de sa phase [16]). Nous discuterons brièvement dans la Section 10 des fonctions d'échelle à décroissance lente, correspondant à des filtres peu réguliers. 
L'étude des produits infinis (2) avec $m_{j}$ dépendant de $j$ est beaucoup plus récente, et a été théorisée sous le nom d'analyse multi-résolution non-stationnaire [10]. La principale motivation de cette étude est qu'elle permet l'obtention de fonctions d'échelle (non-stationnaires) $C^{\infty}$ et à support compact, ce qui n'est pas possible dans le cas stationnaire. (Si $\varphi$ est une fonction d'échelle de classe $C^{N}$, alors le diamètre de son support est au moins $N+2$ ). Les exemples usuels de telles fonctions d'échelle non-stationnaires sont la fonction de Rvachev up $(x-1)$ [26], [11] et la base de Berkolaïko et Novikov [2], [7] qui permettent une approximation spectrale des fonctions régulières.

La fonction de Rvachev up $(x)$ est encore mal connue du public (mathématique) occidental, les principales références étant en russe ou en ukrainien (dont le livre [27] paru en 1979). Nous en rappellerons les principales propriétés dans la Section 5. (Cette section expose les résultats de Rvachev. Nous avons préféré travailler dans $\mathbb{R}$ plutôt que dans $[-1,1])$.

La seconde série de résultats, que nous présentons dans la Section 7, concerne les analyses multi-résolutions quasi-stationnaires. L'idée est d'appliquer tout le mécanisme de l'analyse du comportement asymptotique des suites de filtres d'échelle développée dans la première série de résultats à la suite $m_{j}$ qui intervient dans le produit (2). Nous verrons que si la suite $m_{j}$ converge vers un filtre asymptote $m_{\infty}$, la connaissance des propriétés de $m_{\infty}$ et de sa fonction d'échelle $\varphi_{\infty}$ simplifie grandement l'étude de la convergence du produit infini (2) et de la taille et de la régularité de la fonction d'échelle non-stationnaire ainsi définie. (Avec la Section 3, c'est le principal résultat du papier).

La troisième série de résultats (sections 7 et 8) concerne la construction effective des suites de filtres approximant un filtre donné. La construction est quasiment immédiate pour le cas des filtres associés à des fonctions d'échelle interpolantes, et fait intervenir l'approximation des fonctions par des polynômes de Bernstein. En particulier, nous sommes à même de construire une fonction d'échelle interpolante nonstationnaire, ou "ondelette de Kharkov", jouissant de propriétés d'approximation remarquables ( $c f$. Théorème 4$)$.

Le cas des fonctions d'échelle orthogonales est beaucoup plus complexe, à cause du problème de la phase. Nous essayerons ici de bien poser le problème, en réservant l'éventuelle solution à des travaux ultérieurs. 


\section{Décroissance et convergence rapides dans un espace local de distributions.}

Nous avons regroupé dans cette section quelques lemmes techniques sur la convergence rapide ( $c f$. Définition 2), qui nous seront utiles pour vérifier les qualités d'approximation dans les théorèmes des sections suivantes. Nous avons choisi de présenter ces lemmes dans un cadre axiomatique assez général (ce que nous appelons dans la Définition 1 un espace local de distributions), mais en réalité nous travaillerons avec des espaces simples comme $L^{2}, L^{\infty}, H^{s}$ ou $B_{\infty}^{\alpha, \infty}$ (c'est-à-dire l'espace de Hölder $C^{\alpha}$ si $\alpha>0$ et $\alpha \notin \mathbb{N}$, l'espace de Zygmund $C_{*}^{\alpha}$ si $\alpha \in \mathbb{N}^{*}$ ).

Definition 1. Un espace local de distribution (ou E.L.D.) est un espace de Banach $E$, continûment injecté dans $\mathcal{D}^{\prime}(\mathbb{R})$, tel que

i) pour tout $\varphi \in E$ et tout $\omega \in C_{c}^{\infty}(\mathbb{R}), \omega \varphi \in E$,

ii) il existe $C_{0} \geq 0$ et $N_{0} \in \mathbb{N}$ tel que, pour tout $\varphi \in E$ et tout $\omega \in C_{c}^{\infty}$ avec $\operatorname{supp} \omega \subset[-1,1]$,

$$
\|\varphi \omega\|_{E} \leq C_{0} \sum_{p=0}^{N_{0}}\left\|\frac{d^{p}}{d x^{p}} \omega\right\|_{L^{\infty}([-1,1])}\|\varphi\|_{E}
$$

iii) pour tout $\varphi \in E$ et tout $x_{0} \in \mathbb{R}, \varphi\left(x-x_{0}\right) \in E$ et il existe $C_{1} \geq 0$ et $N_{1} \geq 0$ tel que

$$
\left\|\varphi\left(x-x_{0}\right)\right\|_{E} \leq C_{1}\left(1+\left|x_{0}\right|\right)^{N_{1}}\|\varphi\|_{E},
$$

pour tout $\varphi \in E$ et tout $x_{0} \in \mathbb{R}$.

Definition 2. Soit E un E.L.D.

i) $\varphi$ est à décroissance rapide dans $E$ si pour tout entier $k \in \mathbb{N}$, $x^{k} \varphi \in E$.

ii) Une suite $\left\{\varphi_{n}\right\}_{n \in \mathbb{N}}$ est à convergence rapide dans $E$ si les $\varphi_{n}$ sont $\grave{a}$ décroissance rapide dans $E$, convergent dans $E$ vers une distribution $\varphi$ et pour tout entier $k \in \mathbb{N}, x^{k} \varphi_{n}$ converge dans $E$ vers $x^{k} \varphi$.

On dira de même qu'une série $\sum_{k \in \mathbb{Z}} \varphi_{k}$ est à convergence rapide si les sommes partielles $\left\{\sum_{0}^{n} \varphi_{k}\right\}_{n \in \mathbb{N}}$ et $\left\{\sum_{-n}^{0} \varphi_{k}\right\}_{n \in \mathbb{N}}$ sont à convergence rapide. 
Lemme A (Lemme des blocs). Soit E un E.L.D.

a) Si $\operatorname{supp} \varphi_{k} \subset[k-1, k+1]$ pour tout $k \in \mathbb{Z}$, alors $\sum_{\mathbb{Z}} \varphi_{k}$ est ̀̀ convergence rapide dans $E$ si et seulement si la suite $\left\{\left\|\varphi_{k}\right\|_{E}\right\}_{k \in \mathbb{Z}}$ est $\grave{a}$ décroissance rapide (pour tout $p \in \mathbb{N},\left\{k^{p}\left\|\varphi_{k}\right\|_{E}\right\}_{k \in \mathbb{Z}} \in \ell^{\infty}(\mathbb{Z})$ ).

b) $\varphi$ est à décroissance rapide dans $E$ si et seulement si $\varphi$ se décompose en $\sum_{\mathbb{Z}} \varphi_{k}$ avec $\operatorname{supp} \varphi_{k} \subset[k-1, k+1]$ et $\left\{\left\|\varphi_{k}\right\|_{E}\right\}_{k \in \mathbb{Z}}$ à décroissance rapide.

c) pour qu'une suite $\left\{\varphi_{n}\right\}_{n \in \mathbb{N}}$ converge rapidement dans $E$, il suffit que $\left\{\varphi_{n}\right\}_{n \in \mathbb{N}}$ converge dans $E$ et que pour tout $p \in \mathbb{N}, \sup _{k}\left\|x^{p} \varphi_{k}\right\|_{E}<$ $+\infty$.

Preuve. a) Le lemme est presque évident. Si $\left\{\varphi_{n}\right\}$ converge rapidement dans $E$, il est clair que $\sup _{k}\left\|x^{p} \varphi_{k}\right\|_{E}<+\infty$. En particulier, si $\sum \varphi_{k}$ converge rapidement, avec $\operatorname{supp} \varphi_{k} \subset[k-1, k+1]$, on a $\sup _{k}\left\|x^{p} \varphi_{k}\right\|_{E}<+\infty$. Mais si $\omega$ est $C^{\infty}$, vaut 1 sur $[-1,1]$ et a son support contenu dans $[-2,2]$, on a, pour $|k| \geq 3$,

$$
\varphi_{k}=\omega_{k}(x-k) x^{p} \varphi_{k}(x) \quad \text { avec } \quad \omega_{k}=(x+k)^{-p} \omega(x) .
$$

(4) et (5) permettent de conclure que

$$
\left\|\varphi_{k}\right\|_{E} \leq C_{p}(1+|k|)^{2 N_{1}-p}\left\|x^{p} \varphi_{k}\right\|_{E}
$$

et donc que $\left\{\left\|\varphi_{k}\right\|_{E}\right\}$ est à décroissance rapide. Inversement, si $\left\|\varphi_{k}\right\|_{E}$ est à décroissance rapide, on écrit $x^{p} \varphi_{k}=\tilde{\omega}_{k}(x-k) \varphi_{k}$ avec $\tilde{\omega}_{k}=$ $(x+k)^{p} \omega(x)$; on a alors

$$
\left\|x^{p} \varphi_{k}\right\|_{E} \leq C_{p}(1+|k|)^{2 N_{1}+p}\left\|\varphi_{k}\right\|_{E}
$$

de sorte que $\sum_{k \in \mathbb{Z}}\left\|x^{p} \varphi_{k}\right\|_{E}<+\infty$. La série $\sum x^{p} \varphi_{k}$ converge dans $E$, et converge vers $x^{p} \sum \varphi_{k}$ puisque $E$ s'injecte dans $\mathcal{D}^{\prime}$. Le point a) est donc prouvé.

b) est immédiat: si $\varphi$ est à décroissance rapide, on prend $\gamma \in C_{c}^{\infty}$ avec supp $\gamma \subset[-1,1]$ et $\sum \gamma(x-k)=1$ et on pose $\varphi_{k}=\varphi \gamma(x-k)$. Alors pour $|k| \geq 3$, on a

$$
\left\|\varphi_{k}\right\|_{E}=\left\|\gamma(x-k) x^{-p} x^{p} \varphi\right\|_{E} \leq C_{p}(1+|k|)^{2 N_{1}-p}\left\|x^{p} \varphi\right\|_{E},
$$

et $\left\{\left\|\varphi_{k}\right\|_{E}\right\}$ est à décroissance rapide. 
c) est tout aussi immédiat: si $\varphi_{n}$ converge vers $\varphi, \gamma(x-k) \varphi_{n}$ converge vers $\gamma(x-k) \varphi$; or on a

$$
\left\|\gamma(x-k) \varphi_{n}\right\|_{E} \leq C_{p}(1+|k|)^{2 N_{1}-p}\left\|x^{p} \varphi_{n}\right\|_{E}
$$

Le point c) du Lemme $\mathrm{A}$ se généralise de la manière évident suivante:

Lemme B. Soient $E, E_{1}, E_{2}$ trois E.L.D. tels que $E_{1} \cap E_{2} \subset E$ et, pour tout $f \in E_{1} \cap E_{2}$

$$
\|f\|_{E} \leq C_{2}\|f\|_{E_{1}}^{\alpha}\|f\|_{E_{2}}^{1-\alpha}
$$

pour un $\alpha \in] 0,1\left[\right.$. Alors si $\left\{\varphi_{n}\right\}_{n \in \mathbb{N}}$ converge dans $E_{1}$ et vérifie pour tout $p \in \mathbb{N}, \sup _{n}\left\|x^{p} \varphi_{n}\right\|_{E_{2}}<+\infty,\left\{\varphi_{n}\right\}$ converge rapidement dans $E$.

Preuve. Même démonstration: $\left\{\gamma(x-k) \varphi_{n}\right\}_{n \in \mathbb{N}}$ converge dans $E$ (puisque elle est de Cauchy dans $E_{1}$ et bornée dans $E_{2}$ ); de plus

$$
\left\|\gamma(x-k) \varphi_{n}\right\|_{E_{1}} \leq C(1+|k|)^{2 N_{1}}\left\|\varphi_{n}\right\|_{E_{1}}
$$

et

$$
\left\|\gamma(x-k) \varphi_{n}\right\|_{E_{2}} \leq C_{p}(1+|k|)^{2 N_{2}-p}\left\|x^{p} \varphi_{n}\right\|_{E_{2}}
$$

Lemme C. Soit $E$ un E.L.D. et $\varphi$ à décroissance rapide dans $E$.

a) Si $\varphi=\sum \varphi_{k}$ est une décomposition en blocs de $\varphi$ (i.e. $\operatorname{supp} \varphi_{k}$ $\subset[k-1, k+1]$ et la série converge rapidement dans $E$ ) alors si $b \in$ $C^{\infty}(\mathbb{R})$ est à croissance lente ainsi que toutes ses dérivées (pour tout $p \in$ $\mathbb{N}$, il existe $\left.N \geq 0,(|x|+1)^{-N} d^{p} b / d x^{p} \in L^{\infty}\right)$ la série $\sum_{k \in \mathbb{Z}}\left\langle\varphi_{k}, b\right\rangle$ converge vers une somme indépendante du choix des blocs $\varphi_{k}$ et notée $\langle\varphi, b\rangle$.

b) La transformée de Fourier de $\varphi, \hat{\varphi}(\xi)=\left\langle\varphi, e^{i x \xi}\right\rangle$, est une fonction $C^{\infty}$ à croissance lente ainsi que toutes ses dérivées.

Preuve. Remarquons d'abord que d'après (4) on a: si supp $\omega \subset[-1,1]$ et $\varphi \in E$

$$
|\langle\varphi, \omega\rangle| \leq C\|\varphi\|_{E} \sum_{p=0}^{N}\left\|\frac{d^{p} \omega}{d x^{p}}\right\|_{L^{\infty}(-1,1)}
$$


en effet, on a pour $\psi \in E$ avec $\operatorname{supp} \psi \subset[-1,1],|\langle\psi, 1\rangle| \leq C\|\psi\|_{E}$ et on utilise ceci pour $\psi=\varphi \bar{\omega}$. On en conclut que

$$
\left|\left\langle\varphi_{k}, b\right\rangle\right| \leq C(1+|k|)^{M+2 N_{1}}\left\|\varphi_{k}\right\|_{E} \sum_{p=0}^{N}\left\|(1+|x|)^{-M} \frac{d^{p} b}{d^{p} x}\right\|_{\infty}
$$

de sorte que $\sum\left|\left\langle\varphi_{k}, b\right\rangle\right|$ converge. Le point a) est alors immédiat.

Pour le point b), il suffit de vérifier que $\hat{\varphi}$ est continue, puisque $(d / d \xi)^{N} \hat{\varphi}=\left(\widehat{-i x)^{N}} \varphi\right.$ et que $x^{N} \varphi$ est encore à décroissance rapide dans $E$. Mais $\hat{\varphi}(\xi)=\sum_{k \in \mathbb{Z}} \hat{\varphi}_{k}(\xi)$ et on a

$$
\left|\hat{\varphi}_{k}(\xi)\right| \leq C(1+|k|)^{2 N_{1}}\left\|\varphi_{k}\right\|_{E}(1+|\xi|)^{N},
$$

de sorte que $\sum \hat{\varphi}_{k}$ converge uniformément sur tout compact.

Lemme D (Lemme de dérivation).

a) Soient $E_{1}$ et $E_{2}$ deux E.L.D. tels que $d / d x$ est continu de $E_{1}$ dans $E_{2}$. Alors si $\varphi$ est à décroissance rapide dans $E_{1}, d \varphi / d x$ est $\grave{a}$ décroissance rapide dans $E_{2}$.

b) Le résultat reste vrai si l'on suppose seulement que $d / d x$ est continu de $E_{1,0}=\left\{\varphi \in E_{1}: \operatorname{supp} \varphi \subset[-1,1]\right\}$ dans $E_{2}$.

c) On a de plus

$$
\widehat{d \varphi}(0)=\left\langle\frac{d \varphi}{d x}, 1\right\rangle=0
$$

Preuve. Il suffit de prouver b). On décompose $\varphi$ en blocs $\varphi=\sum \varphi_{k}$. Alors

$$
\frac{d \varphi}{d x}=\sum \frac{d \varphi_{k}}{d x}
$$

dans $\mathcal{D}^{\prime}$. De plus

$$
\left\|\frac{d \varphi_{k}}{d x}\right\|_{E_{2}} \leq C(1+|k|)^{N_{1}+N_{2}}\left\|\varphi_{k}\right\|_{E_{1}},
$$

de sorte que $\left\|d \varphi_{k} / d x\right\|_{E_{2}}$ est à décroissance rapide.

Ce lemme a une réciproque. 
Lemme $\mathbf{E}$ (Lemme de primitivation). Soient $E_{1}$ et $E_{2}$ deux E.L.D. tels que: $\varphi \rightarrow \int_{-\infty}^{x} \varphi(t) d t$ est continu de $E_{2,(0)}=\left\{\varphi \in E_{2}: \operatorname{supp} \varphi \subset\right.$ $[-1,1]$ et $\langle\varphi, 1\rangle=0\}$ dans $E_{1}$. Alors si $\varphi \in E_{2}$ est à décroissance rapide, $\varphi$ peut s'écrire $\varphi=d \omega / d x$ où $\omega$ est à décroissance rapide dans $E_{1}$ si et seulement si $\langle\varphi, 1\rangle=0$.

Preuve. Si $\varphi=d \omega / d x$, on a $\hat{\varphi}=i \xi \hat{\omega}$ donc $\hat{\varphi}(0)=0$. Pour le résultat inverse, il suffit de montrer qu'on peut écrire $\varphi=\sum \varphi_{k}$ une décomposition en blocs avec $\left\langle\varphi_{k}, 1\right\rangle=0$ pour tout $k$. Si $\omega_{k}$ est le primitive à support compact de $\varphi_{k}$, on aura alors

$$
\left\|\omega_{k}\right\|_{E_{1}} \leq C(1+|k|)^{N_{1}+N_{2}}\left\|\varphi_{k}\right\|_{E_{2}}
$$

et donc $\sum \omega_{k}$ sera une décomposition en blocs d'une distribution $\omega$ à décroissance rapide dans $E_{1}$.

On commence par décomposer $\varphi$ en $\varphi=\sum \psi_{k}$, une décomposition en blocs sans condition sur $\psi_{k}$. On prend alors $\alpha \in E, \operatorname{supp} \alpha \subset[0,1]$ et $\langle\alpha, 1\rangle=1$ (un tel $\alpha$ existe si $E \neq\{0\}$ ). On écrit

$$
\psi_{k}=\psi_{k}-\left\langle\psi_{k}, 1\right\rangle \alpha(x-k)+\left\langle\psi_{k}, 1\right\rangle \alpha(x-k) .
$$

La somme

$$
\sum_{k \in \mathbb{Z}} \psi_{k}-\left\langle\psi_{k}, 1\right\rangle \alpha(x-k)
$$

est une décomposition par blocs avec des blocs de somme nulle. Nous sommes ramenés à traiter le cas de

$$
\varphi=\sum_{k \in \mathbb{Z}} \varepsilon_{k} \alpha(x-k)
$$

avec $\left\{\varepsilon_{k}\right\}$ à décroissance rapide et $\sum \varepsilon_{k}=0$. Il suffit d'écrire $\varepsilon_{k}=$ $s_{k}-s_{k+1}$ et

$$
\varphi=\sum_{k \in \mathbb{Z}} s_{k}(\alpha(x-k)-\alpha(x-k+1))
$$

on conclut en remarquant que $\left\{s_{k}\right\}$ est à décroissance rapide puisque

$$
s_{k}=\sum_{k}^{+\infty} \varepsilon_{p}=-\sum_{-\infty}^{k-1} \varepsilon_{p} .
$$


Lemme F (Lemme de primitivation discrète). Soit $E$ un E.L.D. et $\varphi$ à décroissance rapide dans E. Alors les trois propriétés suivantes sont équivalentes:

F1) pour tout $k \in \mathbb{Z}, \hat{\varphi}(2 k \pi)=0$,

F2) $\sum_{k \in \mathbb{Z}} \varphi(x-k)$ converge dans $\mathcal{D}^{\prime}$ vers 0 ,

F3) Il existe $\omega \in E$ à décroissance rapide tel que

$$
\varphi=\omega(x)-\omega(x-1) .
$$

Preuve. Il n'y a presque rien à démontrer. F3) implique F1) est évident, puisqu'alors $\hat{\varphi}=\left(1-e^{-i \xi}\right) \hat{\omega}$. Pour F1) implique F2) et F2) implique F3), on commence par remarquer que si $\varphi$ est à décroissance rapide dans $E$, alors $\sum \varphi(x-k)$ converge dans $\mathcal{D}^{\prime}$ (et même dans $\mathcal{S}^{\prime}$ )

$$
\sum\langle\varphi(x-k), \beta\rangle=\left\langle\varphi, \sum \beta(x+k)\right\rangle
$$

et $\sum \beta(x+k)$ est $C^{\infty}$ bornée ainsi que toutes ses dérivées.

Il reste à vérifier que

$$
\sum \varphi(x-k)=\sum \hat{\varphi}(2 k \pi) e^{i k x},
$$

c'est évident si le support de $\varphi$ est compact. Dans le cas général, on décompose $\varphi$ en blocs $\sum \varphi_{p}$; on a

$$
\sum \varphi(x-k)=\sum \sum \varphi_{p}(x-k),
$$

au sens que pour tout $b \in \mathcal{S}$

$$
\sum \sum\left|\left\langle\varphi_{p}(x-k), b\right\rangle\right|<+\infty .
$$

On a donc

$$
\sum \varphi(x-k)=\sum_{p}\left(\sum_{k} \hat{\varphi}_{p}(2 k \pi) e^{i k x}\right)
$$

et comme

$$
\left|\hat{\varphi}_{p}(2 k \pi)\right| \leq C_{N} \frac{(1+|k|)^{N}}{1+p^{2}}
$$


la convergence dans $\mathcal{D}^{\prime}$ est immédiate.

Si $\sum \varphi(x-k)=0$, on a $\hat{\varphi}(2 k \pi)=0$. Si $\hat{\varphi}(2 k \pi)=0$, on a $\sum \varphi(x-k)=0$; on définit alors

$$
\omega=\sum_{k=0}^{\infty} \varphi(x-k)=-\sum_{k=-\infty}^{-1} \varphi(x-k) .
$$

On a

$$
\|\gamma(x-p) \varphi(x-k)\|_{E} \leq C(1+|k|)^{N_{1}}\|\gamma(x-p+k) \varphi\|_{E},
$$

et on déduit tout de suite que $\omega$ est à décroissance rapide dans $E$.

\section{La théorie classique des fonctions d'échelle.}

La notion de fonction d'échelle régulière a été introduite en 1986 par Y. Meyer et S. Mallat [20], [21].

Definition 3. Une fonction d'échelle régulière est une fonction $\varphi \in$ $L^{2}(\mathbb{R})$ telle que

i) $\varphi$ est à valeurs réelles,

ii) $\varphi$ est à décroissance rapide dans $L^{2}$ : pour tout $k \in \mathbb{Z}, x^{k} \varphi \in L^{2}$,

iii) $\varphi$ engendre une base de Riesz $\{\varphi(x-k)\}_{k \in \mathbb{Z}}$ d'un sous-espace fermé $V_{0}$ de $L^{2}$,

iv) $\varphi(x / 2) \in V_{0}$.

L'étude des fonctions d'échelle se ramène à celle des filtres d'échelle: l'appartenance de $\varphi(x / 2)$ à $V_{0}$ se réécrit en

$$
\varphi\left(\frac{x}{2}\right)=\sum_{k \in \mathbb{Z}} a_{k} \varphi(x-k),
$$

où les $a_{k}$ sont à valeurs réelles et dans $\ell^{2}(\mathbb{Z})$. Le filtre d'échelle associé à $\varphi$ est la fonction $2 \pi$-périodique $m_{0}$ définie par

$$
m_{0}(\xi)=\frac{1}{2} \sum_{k \in \mathbb{Z}} a_{k} e^{-i k \xi} .
$$


Quelques lemmes classiques permettent alors de se ramener à l'étude des propriétés de $m_{0}$.

Lemme 1. Si $\varphi$ et $x \varphi$ sont de carré intégrable, alors

$$
\sum_{k \in \mathbb{Z}}|\hat{\varphi}(\xi+2 k \pi)|^{2}
$$

converge uniformément sur $[-\pi, \pi]$.

Preuve. $|\hat{\varphi}|^{2} \in L^{1}$ donc

$$
\sum_{k \in \mathbb{Z}}|\hat{\varphi}(\xi+2 k \pi)|^{2}
$$

converge presque partout, donc en au moins un point $\xi_{0}$. Il suffit alors d'écrire pour $\xi \in\left[\xi_{0}-\pi, \xi_{0}+\pi\right]$

$$
\begin{aligned}
\left|\left(\sum_{|k|>K}|\hat{\varphi}(\xi+2 k \pi)|^{2}\right)^{1 / 2}-\left(\sum_{|k|>K}\left|\hat{\varphi}\left(\xi_{0}+2 k \pi\right)\right|^{2}\right)^{1 / 2}\right| \\
\leq\left(\sum_{|k|>K}\left|\hat{\varphi}(\xi+2 k \pi)-\hat{\varphi}\left(\xi_{0}+2 k \pi\right)\right|^{2}\right)^{1 / 2} \\
\leq\left(\sum_{|k|>K}\left|\xi-\xi_{0}\right| \int_{\xi_{0}+2 k \pi}^{\xi+2 k \pi}\left|\frac{d \hat{\varphi}}{d \eta}(\eta)\right|^{2} d \eta\right)^{1 / 2} \\
\leq\left|\xi-\xi_{0}\right|^{1 / 2}\left(\int_{\left|\eta-\xi_{0}\right|>2 K \pi-\pi}\left|\frac{d}{d \eta} \hat{\varphi}(\eta)\right|^{2} d \eta\right)^{1 / 2}
\end{aligned}
$$

\section{Lemme 2.}

i) Si $\varphi$ et $x \varphi$ sont de carré intégrable, alors $\{\varphi(x-k)\}_{k \in \mathbb{Z}}$ est une base de Riesz d'un sous-espace fermé de $L^{2}$ si et seulement si $\sum_{k \in \mathbb{Z}}|\hat{\varphi}(\xi+2 k \pi)|^{2}$ ne s'annule pas sur $[-\pi, \pi]$.

ii) La fonction $m_{0}$, filtre d'échelle associé à une fonction d'échelle régulière $\varphi$, est $C^{\infty}$ et vérifie $\hat{\varphi}(2 \xi)=m_{0}(\xi) \hat{\varphi}(\xi)$.

Preuve. Le point i) est évident: si $\varphi \in L^{2}$, le fait que $\{\varphi(x-k)\}_{k \in \mathbb{Z}}$ soit une famille de Riesz est équivalent à ce que

$$
\inf \operatorname{ess} \sum_{k \in \mathbb{Z}}|\hat{\varphi}(\xi+2 k \pi)|^{2}>0
$$


et que

$$
\sup \operatorname{ess} \sum_{k \in \mathbb{Z}}|\hat{\varphi}(\xi+2 k \pi)|^{2}<+\infty,
$$

si de plus $x \varphi \in L^{2}$, alors

$$
\sum_{k \in \mathbb{Z}}|\hat{\varphi}(\xi+2 k \pi)|^{2}
$$

est continue. Pour ii), il suffit de remarquer que

$$
m_{0}(\xi)=\frac{\sum_{k \in \mathbb{Z}} \hat{\varphi}(2 \xi+4 k \pi) \hat{\varphi}(\xi+2 k \pi)}{\sum_{k \in \mathbb{Z}}|\hat{\varphi}(\xi+2 k \pi)|^{2}}=\frac{\sum_{k \in \mathbb{Z}}\left\langle\varphi\left(\frac{x}{2}\right), \varphi(x-k)\right\rangle e^{-i k \xi}}{\sum_{k \in \mathbb{Z}}\langle\varphi(x), \varphi(x-k)\rangle e^{-i k \xi}}
$$

et que les coefficients $\langle\varphi(x / 2), \varphi(x-k)\rangle$ et $\langle\varphi(x), \varphi(x-k)\rangle$ sont à décroissance rapide.

Lemme 3. Si $\varphi$ est une fonction d'échelle régulière, de filtre $m_{0}$, alors $m_{0}(0)=1, \hat{\varphi}(0) \neq 0$ et

$$
\hat{\varphi}(\xi)=\hat{\varphi}(0) \prod_{j=1}^{\infty} m_{0}\left(\frac{\xi}{2^{j}}\right)
$$

Preuve. On remarque que $\left|m_{0}(0)\right| \leq 1$

$$
\sum_{k \in \mathbb{Z}}|\hat{\varphi}(4 k \pi)|^{2}=\left|m_{0}(0)\right|^{2} \sum_{k \in \mathbb{Z}}|\hat{\varphi}(2 k \pi)|^{2} \text {. }
$$

On a alors

$$
|\hat{\varphi}(\xi)|=\prod_{j=1}^{N}\left|m_{0}\left(\frac{\xi}{2^{j}}\right)\right|\left|\hat{\varphi}\left(\frac{\xi}{2^{N}}\right)\right|=|\hat{\varphi}(0)| \prod_{j=1}^{\infty}\left|m_{0}\left(\frac{\xi}{2^{j}}\right)\right| .
$$

La convergence du produit infini est en effet immédiate: si $\left|m_{0}(0)\right|<1$, il converge vers 0 , si $\left|m_{0}(0)\right|=1$, on a $\left|m_{0}(\xi)\right|=1+O\left(\xi / 2^{j}\right)$. On en conclut que $\hat{\varphi}(0) \neq 0$, donc que $m_{0}(0)=1$ et enfin que

$$
\hat{\varphi}(\xi)=\hat{\varphi}(0) \prod_{j=1}^{\infty} m_{0}\left(\frac{\xi}{2^{j}}\right) .
$$


Lemme 4. Si $m_{0} \in C^{\infty}(\mathbb{R} / 2 \pi \mathbb{Z})$ et $m_{0}(0)=1$, alors la fonction

$$
\prod_{j=1}^{\infty} m_{0}\left(\frac{\xi}{2^{j}}\right)
$$

est $C^{\infty}$

Preuve. Comme

$$
\hat{\varphi}(\xi)=\prod_{j=1}^{\infty} m_{0}\left(\frac{\xi}{2^{j}}\right)
$$

vérifie

$$
\hat{\varphi}(\xi)=\prod_{j=1}^{N} m_{0}\left(\frac{\xi}{2^{j}}\right) \hat{\varphi}\left(\frac{\xi}{2^{N}}\right),
$$

il suffit de le vérifier sur un voisinage assez petit de 0 . Mais si $\xi$ est assez petit, $\operatorname{Re} m_{0}\left(\xi / 2^{j}\right)>0$ pour tout $j$ et on peut passer au logarithme

$$
\hat{\varphi}(\xi)=e^{\sum_{j=1}^{\infty} \log m_{0}\left(\xi / 2^{j}\right)} .
$$

Maintenant, si $\theta$ est définie sur $[-\varepsilon, \varepsilon](\varepsilon>0), C^{\infty} \operatorname{sur}[-\varepsilon, \varepsilon]$ et si $\theta(0)=0$ alors $\sum_{j \geq 1} \theta\left(\xi / 2^{j}\right)$ est $C^{\infty}$ sur $[-\varepsilon, \varepsilon]$ : la convergence des séries dérivées est immédiate et celle de la série de départ s'obtient par

$$
\left|\theta\left(\frac{\xi}{2^{j}}\right)\right| \leq C \frac{|\xi|}{2^{j}}
$$

Les lemmes 3 et 4 ramènent donc l'étude des fonctions d'échelle régulières à celle des filtres d'échelle. Les filtres d'échelle ont été caractérisés par de nombreux travaux (nous utiliserons essentiellement [4], [6] et [14]). Une conséquence immédiate du Lemme 2 est que si $\varphi$ est une fonction d'échelle régulière alors $\hat{\varphi}$ ne peut avoir de zéro $2 \pi$-périodique; cela se caractérise facilement sur le filtre $m_{0}$ : c'est le critère d'Albert Cohen.

Definition 4. Une fonction $m_{0} \in C^{\infty}(\mathbb{R} / 2 \pi \mathbb{Z})$ telle que $m_{0}(0)=1$ satisfait le critère d'Albert Cohen s'il existe un compact $K$ réunion finie d'intervalles fermés tel que 0 est un point intérieur à $K$ et tel que

$$
\sum_{k \in \mathbb{Z}} \chi_{K}(\xi+2 k \pi)=1, \quad \text { presque partout }
$$


où

$$
\chi_{K}(x)= \begin{cases}1, & \text { si } x \in K, \\ 0, & \text { si } x \notin K .\end{cases}
$$

Pour tous $\xi \in K, j \geq 1$,

$$
m_{0}\left(\frac{\xi}{2^{j}}\right) \neq 0
$$

Un tel compact est appelé compact d'Albert Cohen associé à $m_{0}$.

Le rôle de ce critère est explicité par le lemme suivant

Lemme 5. Soit $m_{0} \in C^{\infty}(\mathbb{R} / 2 \pi \mathbb{Z})$ telle que $m_{0}(0)=1$ et soit

$$
\hat{\varphi}=\prod_{j=1}^{\infty} m_{0}\left(\frac{\xi}{2^{j}}\right) .
$$

i) $\hat{\varphi}$ n'a pas de zéro $2 \pi$-périodique si et seulement si $m_{0}$ vérifie le critère d'Albert Cohen.

ii) Dans ce cas, si $K$ est un compact d'Albert Cohen associé à $m_{0}$, on a $\inf _{\eta \in K}|\hat{\varphi}(\eta)|>0$. De plus les fonctions

$$
\theta_{N}(\xi)=\chi_{K}\left(\frac{\xi}{2^{N}}\right) \prod_{j=1}^{N} m_{0}\left(\frac{\xi}{2^{j}}\right)
$$

convergent ponctuellement vers $\hat{\varphi}$ et sont dominées par $\hat{\varphi}$

$$
\left|\theta_{N}(\xi)\right| \leq \frac{1}{\inf _{\eta \in K}|\hat{\varphi}(\eta)|}|\hat{\varphi}(\xi)| .
$$

En particulier si $1 \leq p<+\infty$ et $w \in L_{\mathrm{loc}}^{1}$ avec $w(x)>0$, les trois assertions suivantes sont équivalentes:

j) $\theta_{N} \rightarrow \hat{\varphi}$ dans $L^{p}(w d x)$ quand $N \rightarrow+\infty$,

jj) $\hat{\varphi} \in L^{p}(w d x)$,

jjj) $\sup _{N \geq 1}\left\|\theta_{N}\right\|_{L^{p}(w d x)}<+\infty$.

Preuve. Ce lemme est évident. Si $\hat{\varphi}$ n'a pas de zéro $2 \pi$-périodique, on note $\mathcal{I}_{0}$ la collection des intervalles sur lesquels $\hat{\varphi}$ ne s'annule pas, et $\mathcal{I}$ 
la collection des intervalles $I$ de la forme $I=I_{0}+2 k \pi, I_{0} \in \mathcal{I}_{0}, k \in \mathbb{Z}$; on a clairement $[-\pi, \pi] \subset \bigcup_{I \in \mathcal{I}} I$ et le compact $K$ se construit à l'aide d'un sous-recouvrement fini de $[-\pi, \pi]$.

Inversement, si $K$ est un compact d'Albert Cohen pour $m_{0}, \hat{\varphi}$ ne s'annule pas sur $K$ (puisqu'aucun des termes $m_{0}\left(\xi / 2^{j}\right)$ ne s'y annule) et y est continue; on a donc $\min _{\xi \in K}|\hat{\varphi}(\xi)|>0$. Maintenant, si $\xi \in$ $\mathbb{R}$, il existe nécessairement $k \in \mathbb{Z}$ tel que $\hat{\varphi}(\xi+2 k \pi) \in K$ : en effet $\cup_{k \in \mathbb{Z}} K+2 k \pi$ est localement fermée, donc fermée, donc coïncide avec $\mathbb{R}$ tout entier (puisque $\left.\mid \mathbb{R} \backslash \cup_{\mathbb{Z}} K+2 k \pi\right) \mid=0$ ). Le reste du lemme est alors immédiat, puisque

$$
\theta_{N}(\xi)= \begin{cases}\frac{\hat{\varphi}(\xi)}{\hat{\varphi}\left(\frac{\xi}{2^{N}}\right)}, & \text { si } \xi \in 2^{N} K \\ 0, & \text { si } \xi \notin 2^{N} K\end{cases}
$$

Le résultat classique principal est alors le suivant [6], [14].

Proposition 1. Soit $m_{0} \in C^{\infty}(\mathbb{R} / 2 \pi \mathbb{Z})$ telle que $m_{0}(0)=1$. On note $\hat{\varphi}$ la fonction

$$
\hat{\varphi}(\xi)=\prod_{j=1}^{\infty} m_{0}\left(\frac{\xi}{2^{j}}\right)
$$

et $T_{2}$ l'opérateur agissant sur les fonctions $2 \pi$-périodiques défini par

$$
T_{2} f=\left|m_{0}\left(\frac{\xi}{2}\right)\right|^{2} f\left(\frac{\xi}{2}\right)+\left|m_{0}\left(\frac{\xi}{2}+\pi\right)\right|^{2} f\left(\frac{\xi}{2}+\pi\right) .
$$

Alors les deux assertions suivantes sont équivalentes:

A1) $\hat{\varphi}$ est la transformée de Fourier d'une fonction d'échelle régulière,

A2) $m_{0}$ satisfait les trois conditions suivantes:

i) $m_{0}(\xi)=\overline{m_{0}(-\xi)}$,

ii) $m_{0}$ satisfait le critère d'Albert Cohen,

iii) $\sup _{N \in \mathbb{N}}\left\|T_{2}^{N}(1)\right\|_{\infty}<+\infty$.

De plus, lorsque A1) ou A2) sont vérifiées, il existe $\alpha>0$ tel que $\varphi \in H^{\alpha}$ (i.e. tel que $|\xi|^{\alpha} \hat{\varphi} \in L^{2}$ ). 
Preuve. A1) implique A2) est évident: i) vient de ce que $\varphi$ est à valeurs réelles, ii) de ce que $\hat{\varphi}$ n'a pas de zéro $2 \pi$-périodique, enfin iii) vient de ce que

$$
\gamma(\xi)=\sum_{k \in \mathbb{Z}}|\hat{\varphi}(\xi+2 k \pi)|^{2}
$$

ne s'annule pas et de ce que $T_{2}$ est un opérateur positif

$$
1 \leq \frac{1}{\inf _{|\eta| \leq \pi} \gamma(\eta)} \gamma(\xi)
$$

et $T_{2}(\gamma)=\gamma$, de sorte que

$$
T_{2}^{N}(1)(\xi) \leq \frac{1}{\inf _{|\eta| \leq \pi} \gamma(\eta)} T_{2}^{N}(\gamma)(\xi)=\frac{\gamma(\xi)}{\inf _{|\eta| \leq \pi} \gamma(\eta)}
$$

A2) implique A1): On va commencer par montrer qu'il existe un $\rho_{0} \in$ ]0, $1[$ tel que

$$
\sup _{\xi \in[-\pi, \pi]} \sup _{N \in \mathbb{N}} \rho_{0}^{-N} \sum_{2^{N} \pi \leq|\xi+2 k \pi|<2^{N+1} \pi}|\hat{\varphi}(\xi+2 k \pi)|^{2}<+\infty .
$$

Cela implique en particulier que $\hat{\varphi}$ appartient à $H^{\alpha}$ dès que $2^{\alpha} \rho_{0}<1$ (i.e. pour tout $\left.\alpha<\log \left(1 / \rho_{0}\right) / \log 2\right)$.

Pour cela, on note

$$
I_{N}(\xi)=\sum_{2^{N} \pi \leq|\xi+2 k \pi|<2^{N+1} \pi}|\hat{\varphi}(\xi+2 k \pi)|^{2} .
$$

On a, puisque $\hat{\varphi}(\xi)=m_{0}(\xi / 2) \hat{\varphi}(\xi / 2)$,

$$
\begin{aligned}
I_{N}(\xi) & =\left|m_{0}\left(\frac{\xi}{2}\right)\right|^{2} I_{N-1}\left(\frac{\xi}{2}\right)+\left|m_{0}\left(\frac{\xi}{2}+\pi\right)\right|^{2} I_{N-1}\left(\frac{\xi}{2}+\pi\right) \\
& =T_{2}\left(I_{N-1}\right)(\xi)
\end{aligned}
$$

et donc

$$
I_{N}(\xi)=T_{2}^{N}\left(I_{0}(\xi)\right)
$$

De même, on note

$$
J_{N}(\xi)=\sum_{|\xi+2 k \pi|<2^{N} \pi}|\hat{\varphi}(\xi+2 k \pi)|^{2}
$$


et on a $J_{N}=T_{2}^{N}\left(J_{0}\right)$, d'où

$$
\left\|J_{N}(\xi)\right\|_{\infty} \leq\left\|J_{0}\right\|_{\infty}\left\|T_{2}^{N}(1)\right\|_{\infty},
$$

ce qui prouve

$$
\sup _{\xi} \sum_{k \in \mathbb{Z}}|\hat{\varphi}(\xi+2 k \pi)|^{2}<+\infty
$$

En particulier, $\hat{\varphi} \in L^{2}$ et les $\varphi(x-k), k \in \mathbb{Z}$, engendrent une base de Riesz d'un sous-espace fermé $V_{0}$ de $L^{2}$ (car

$$
\sum_{k \in \mathbb{Z}}|\hat{\varphi}(\xi+2 k \pi)|^{2} \geq \inf _{\eta \in K}|\hat{\varphi}(\eta)|^{2}>0
$$

pour $K$ un compact d'Albert Cohen associé à $\left.m_{0}\right)$. De plus,

$$
\sum_{k \in \mathbb{Z}}|\hat{\varphi}(2 k \pi)|^{2}=\sum_{k \in \mathbb{Z}}|\hat{\varphi}(2 k \pi)|^{2}+\left|m_{0}(\pi)\right|^{2} \sum_{k \in \mathbb{Z}}|\hat{\varphi}(\pi+2 k \pi)|^{2},
$$

ce qui prouve que $m_{0}(\pi)=0\left(\operatorname{car} \sum_{k \in \mathbb{Z}}|\hat{\varphi}(\pi+2 k \pi)|^{2}>0\right)$, et donc que $\hat{\varphi}(2 k \pi)=0$ pour $k \in \mathbb{Z}^{*}$. On en conclut que $I_{0}(\xi)$ vérifie, pour $|\xi| \leq \pi$

$$
I_{0}(\xi)=\sum_{\pi \leq|\xi+2 k \pi| \leq 2 \pi}|\hat{\varphi}(\xi+2 k \pi)|^{2} \leq C|\xi| \leq C \pi\left|\sin \frac{\xi}{2}\right|,
$$

de sorte que

$$
I_{N}(\xi) \leq C \pi T_{2}^{N}\left(\left|\sin \frac{\xi}{2}\right|\right)
$$

Il reste à estimer

$$
\left\|T_{2}^{N}\left(\left|\sin \frac{\xi}{2}\right|\right)\right\|_{\infty}
$$

On remarque d'abord que $T_{2}$ laisse invariant

$$
E_{0}=\left\{f \in C^{0}(\mathbb{R} / 2 \pi \mathbb{Z}): \frac{d f}{d \xi} \in L^{\infty} \text { et } f(0)=0\right\} .
$$

De plus, si $f \in E_{0}$, on a

$$
\left\|T_{2}^{N}(f)\right\|_{\infty} \leq\left\|T_{2}^{N}(1)\right\|_{\infty}\|f\|_{\infty} \leq C\|f\|_{\infty}
$$


et

$$
\begin{aligned}
\left\|\frac{d}{d \xi} T_{2}^{N}(f)\right\|_{\infty} \leq & \left\|\frac{d}{d \xi}\left|m_{0}\right|^{2}\right\|_{\infty}\left\|T_{2}^{N-1} f\right\|_{\infty}+\frac{1}{2}\left\|T_{2}\left(\left|\frac{d}{d \xi} T_{2}^{N-1} f\right|\right)\right\|_{\infty} \\
\leq & \frac{1}{2^{N}}\left\|T_{2}^{N}\left(\left|\frac{d f}{d \xi}\right|\right)\right\|_{\infty} \\
& +\sum_{k=1}^{N}\left\|\frac{d}{d \xi}\left|m_{0}\right|^{2}\right\|_{\infty}\left\|T_{2}^{N-k} f\right\|_{\infty}\left\|T_{2}^{k-1}(1)\right\|_{\infty}\left(\frac{1}{2}\right)^{k-1} \\
\leq & C\left(\|f\|_{\infty}+\frac{1}{2^{N}}\left\|\frac{d f}{d \xi}\right\|_{\infty}\right) .
\end{aligned}
$$

En particulier, on obtient

(13) $\left\|\frac{d}{d \xi} T_{2}^{N+M}(f)\right\|_{\infty} \leq C_{0}\left(\left\|T_{2}^{N}(f)\right\|_{\infty}+\frac{1}{2^{M}}\left(\|f\|_{\infty}+\left\|\frac{d f}{d \xi}\right\|_{\infty}\right)\right)$,

où $C_{0}$ ne dépend ni de $f$, ni de $N$, ni de $M$.

Par ailleurs, on obtient également que $\left\{T_{2}^{N}(|\sin (\xi / 2)|)\right\}_{N \in \mathbb{N}}$ est bornée dans $E_{0}$, et le théorème d'Ascoli nous assure qu'il existe une sous-suite $\omega_{N_{k}}=T_{2}^{N_{k}}(|\sin (\xi / 2)|)$ qui converge dans $C^{0}(\mathbb{R} / 2 \pi \mathbb{Z})$ vers une fonction $\omega$. On considère alors $K$ un compact d'Albert Cohen associé à $m_{0}$. La fonction

$$
\theta_{n}(\xi)=\sqrt{\left|\sin \frac{\xi}{2^{n+1}}\right|} \chi_{K}\left(\frac{\xi}{2^{n}}\right) \prod_{j=1}^{n} m_{0}\left(\frac{\xi}{2^{j}}\right),
$$

converge ponctuellement vers $\sqrt{\sin (0)} \hat{\varphi}(0)=0$ et se majore par $\hat{\varphi}(\xi) /$ $\inf _{\eta \in K}|\hat{\varphi}(\eta)|$. Comme $\hat{\varphi} \in L^{2}$, le théorème de convergence dominée donne $\theta_{n} \rightarrow 0$ dans $L^{2}$; or

$$
\int\left|\theta_{n}\right|^{2} d \xi=\int_{-\pi}^{\pi} T_{2}^{n}\left(\left|\sin \frac{\xi}{2}\right|\right) d \xi
$$

de sorte que $\int_{-\pi}^{\pi} \omega d x=0$, et donc $\omega=0$ (puisque $\omega \geq 0$ ).

On choisit dans (13) $N=N_{k}$ assez grand pour que

$$
\left\|T_{2}^{N}\left(\left|\sin \frac{\xi}{2}\right|\right)\right\|_{\infty}
$$

soit inférieur à $1 /\left(4 \pi C_{0}\right)$ et $M$ assez grand pour que $3 /\left(2 \cdot 2^{M}\right)$ soit inférieur à $1 /\left(4 \pi C_{0}\right)$. On obtient alors, en posant $P=N+M$

$$
\left\|\frac{d}{d \xi} T_{2}^{P}\left(\left|\sin \frac{\xi}{2}\right|\right)\right\|_{\infty} \leq \frac{1}{2 \pi}
$$


d'où, pour tout $\xi \in[-\pi, \pi]$,

$$
T_{2}^{P}\left(\left|\sin \frac{\xi}{2}\right|\right) \leq \frac{1}{2 \pi}|\xi| \leq \frac{1}{2}\left|\sin \frac{\xi}{2}\right| .
$$

Maintenant, pour $Q \in \mathbb{N}$ quelconque, on obtient

$$
\left\|T_{2}^{Q}\left(\left|\sin \frac{\xi}{2}\right|\right)\right\|_{\infty} \leq\left(\frac{1}{2}\right)^{Q / P} \max _{0 \leq r<P}\left\|T_{2}^{r}\left(\left|\sin \frac{\xi}{2}\right|\right)\right\|_{\infty} 2^{r / P} .
$$

Cela donne finalement: $\left|I_{N}(\xi)\right| \leq C \rho_{0}^{N}$ avec $\rho_{0}=(1 / 2)^{1 / P}$. Nous avons donc démontré (11).

Il ne reste plus à vérifier que la décroissance rapide de $\varphi$ dans $L^{2}$, ou encore que $(d / d \xi)^{p} \hat{\varphi} \in L^{2}$ pour tout $p \in \mathbb{N}$. On va en fait montrer quelque chose de plus fort: il existe $\left.\rho_{p} \in\right] 0,1[$ tel que

$$
\sup _{\xi} \sup _{N \in \mathbb{N}} \rho_{p}^{-N} \sum_{2^{N} \pi \leq|\xi+2 k \pi|<2^{N+1} \pi}\left|\hat{\varphi}^{(p)}(\xi+2 k \pi)\right|^{2}<+\infty .
$$

La démonstration de (14) se fait par récurrence sur $p$. Le cas $p=0$ a été traité avec l'inégalité (11). On va montrer que $\left\|I_{N, p}\right\|_{\infty} \leq C_{p} \rho_{p}^{N}$ où

$$
I_{N, p}(\xi)=\sum_{2^{N} \pi \leq|\xi+2 k \pi|<2^{N+1} \pi}\left|\hat{\varphi}^{(p)}(\xi+2 k \pi)\right|^{2} .
$$

Pour cela, il suffit d'écrire, pour $p \geq 1$,

$$
\hat{\varphi}^{(p)}(\xi)=\sum_{k=0}^{p} C_{p}^{k} \frac{1}{2^{p}} m_{0}^{(p)}\left(\frac{\xi}{2}\right) \hat{\varphi}^{(p+k)}\left(\frac{\xi}{2}\right) .
$$

On utilise l'inégalité

$$
\left(\sum_{k=0}^{p}\left|\alpha_{k}\right|\right)^{2} \leq 2\left|\alpha_{0}\right|^{2}+2\left(\sum_{1}^{p}\left|\alpha_{k}\right|^{2}\right)^{2} \leq 2\left|\alpha_{0}\right|^{2}+2 p \sum_{1}^{p}\left|\alpha_{k}\right|^{2},
$$

et on obtient

$$
\begin{aligned}
I_{N, p}(\xi) \leq & \frac{1}{2^{2 p-1}} T_{2}\left(I_{N-1, p}(\xi)\right) \\
& +2 p \sum_{k=1}^{p} \frac{\left(C_{k}^{p}\right)^{2}}{4^{p}}\left(\left|m_{0}^{(k)}\left(\frac{\xi}{2}\right)\right|^{2} I_{N-1, p-k}\left(\frac{\xi}{2}\right)\right. \\
& \left.\quad+\left|m_{0}^{(k)}\left(\frac{\xi}{2}+\pi\right)\right|^{2} I_{N-1, p-k}\left(\frac{\xi}{2}\right)\right) \\
\leq & \frac{1}{2^{2 p-1}} T_{2}\left(I_{N-1, p}(\xi)\right)+C_{p}^{\prime} \sigma_{p}^{N-1},
\end{aligned}
$$


avec $\sigma_{p}=\max _{0 \leq q \leq p-1} \rho_{q}$ et

$$
C_{p}^{\prime}=\frac{p^{2}}{4^{p-1}}\left(C_{p}^{p / 2}\right)^{2} \max _{0 \leq q \leq p-1}\left(C_{q}\left\|m_{0}^{(q)}\right\|_{\infty}\right) .
$$

Comme $T_{2}$ est un opérateur positif, en itérant $N$ fois on obtient

$I_{N, p}(\xi) \leq\left(\frac{1}{2^{2 p-1}}\right)^{N} T_{2}^{N}\left(I_{0, p}(\xi)\right)+\sum_{k=1}^{N} C_{p}^{\prime} \sigma_{p}^{N-k}\left(\frac{1}{2^{2 p-1}}\right)^{k-1} T_{2}^{k-1}(1)(\xi)$.

Or on contrôle $\left\|T_{2}^{k}(1)\right\|_{\infty}$, et de même

$$
\left\|T_{2}^{N}\left(I_{0, p}(\xi)\right)\right\|_{\infty} \leq\left\|T_{2}^{N}(1)\right\|_{\infty}\left\|I_{0, p}\right\|_{\infty},
$$

de sorte que

$$
I_{N, p}(\xi) \leq C_{p}^{\prime \prime}\left(\frac{1}{\left(2^{2 p-1}\right)^{N}}+\sum_{1}^{N} \sigma_{p}^{N-k}\left(\frac{1}{2^{2 p-1}}\right)^{k-1}\right)
$$

d'où en choisissant $\rho_{p}$ dans $] \max \left(\sigma_{p}, 1 / 2^{2 p-1}\right), 1[$

$$
I_{N, p}(\xi) \leq C_{p}^{\prime \prime} \rho_{p}^{N}\left(1+\sum_{1}^{N}\left(\frac{\sigma_{p}}{\rho_{p}}\right)^{N-k}\right) \leq C_{p}^{\prime \prime \prime} \rho_{p}^{N}
$$

(14) est donc démontrée, ainsi que la Proposition 1.

La démonstration de la Proposition 1, qui donne l'appartenance de $\varphi$ à $H^{\alpha}$ pour un $\alpha>0$, peut être facilement adaptée pour l'étude de l'appartenance de $\varphi$ à $H^{s}, s \in \mathbb{R}$. Parmi les multiples caractérisations, nous en choisissons une qui sera particulièrement bien adaptée à notre étude de la convergence des filtres.

Proposition 2. Soit $m_{0} \in C^{\infty}(\mathbb{R} / 2 \pi \mathbb{Z})$ telle que $m_{0}(0)=1$ et qui satisfait le critère d'Albert Cohen. Soit $\hat{\varphi}=\prod_{j=1}^{\infty} m_{0}\left(\xi / 2^{j}\right)$ et $T_{2}$ l'opérateur

$$
T_{2} f(\xi)=\left|m_{0}\left(\frac{\xi}{2}\right)\right|^{2} f\left(\frac{\xi}{2}\right)+\left|m_{0}\left(\frac{\xi}{2}+\pi\right)\right|^{2} f\left(\frac{\xi}{2}+\pi\right) .
$$

Soit enfin $s \in \mathbb{R}$. Alors les assertions suivantes sont équivalentes: 
B1) Il existe $\sigma>s$ tel que $\varphi \in H^{\sigma}$.

B2) Il existe un entier $N \geq 0$ tel que $N>s$, un nombre $\rho$ tel que $\rho<4^{N-s}$ et un entier $Q \geq 1$ tel que: $C^{\infty}(\mathbb{R} / 2 \pi \mathbb{Z})$,

i) $m_{0}$ se factorise en $m_{0}(\xi)=\left(\left(1+e^{-i \xi}\right) / 2\right)^{N} \tilde{m}_{0}(\xi)$ avec $\tilde{m}_{0} \in$

ii) l'opérateur $\tilde{T}_{2}$, défini par

$$
\tilde{T}_{2} f=\left|\tilde{m}_{0}\left(\frac{\xi}{2}\right)\right|^{2} f\left(\frac{\xi}{2}\right)+\left|\tilde{m}_{0}\left(\frac{\xi}{2}+\pi\right)\right|^{2} f\left(\frac{\xi}{2}+\pi\right)
$$

vérifie

$$
\left\|\tilde{T}_{2}^{Q}(1)\right\|_{\infty} \leq \rho^{Q}
$$

Preuve. B2) implique B1) est relativement immédiat. On a

$$
\begin{aligned}
\int_{|\xi| \geq \pi}|\xi|^{2 \sigma}|\hat{\varphi}(\xi)|^{2} d \xi & \\
& \leq \sum_{k=0}^{\infty} \int_{2^{k} \pi \leq|\xi|<2^{k+1} \pi}\left(2^{k+1} \pi\right)^{2 \sigma} \sup _{|\eta| \leq \pi / 2}|\hat{\varphi}(\eta)|^{2} \prod_{j=1}^{k+1}\left|m_{0}\left(\frac{\xi}{2^{j}}\right)\right|^{2} d \xi \\
& \leq \sum_{k=0}^{\infty}\left(2^{k+1} \pi\right)^{2 \sigma} \sup _{|\eta| \leq \pi}|\hat{\varphi}(\eta)|^{2} \\
& \cdot \int_{|\xi|<2^{k+1} \pi}\left|\sin \frac{\xi}{2^{k+2}}\right|^{2 N} 2^{N} \prod_{j=1}^{k+1}\left|m_{0}\left(\frac{\xi}{2^{j}}\right)\right|^{2} d \xi \\
= & \sum_{k=0}^{\infty}\left(2^{k+1} \pi\right)^{2 \sigma} 2^{N} \sup _{|\eta| \leq \pi}|\hat{\varphi}(\eta)|^{2} \int_{-\pi}^{\pi} T_{2}^{k+1}\left(\sin ^{2 N} \frac{\xi}{2}\right) d \xi
\end{aligned}
$$

Mais on a

$$
T_{2}\left(\sin ^{2 N} \frac{\xi}{2} f\right)=\frac{1}{2^{2 N}} \sin ^{2 N} \frac{\xi}{2} \tilde{T}_{2} f
$$

en effet,

$$
\left|m_{0}\left(\frac{\xi}{2}\right)\right|^{2}=\cos ^{2 N} \frac{\xi}{4}\left|\tilde{m}_{0}\left(\frac{\xi}{2}\right)\right|^{2}
$$

et il suffit d'écrire

$$
\begin{aligned}
\sin ^{2 N} \frac{\xi}{2} & =2^{2 N} \sin ^{2 N} \frac{\xi}{4} \cos ^{2 N} \frac{\xi}{4} \\
& =2^{2 N} \sin ^{2 N}\left(\frac{\xi}{4}+\frac{\pi}{2}\right) \cos ^{2 N}\left(\frac{\xi}{4}+\frac{\pi}{2}\right) .
\end{aligned}
$$


On obtient donc

$$
\int_{-\pi}^{\pi} T_{2}^{k+1}\left(\sin ^{2 N} \frac{\xi}{2}\right) d \xi=\left(\frac{1}{2^{2 N}}\right)^{k+1} \int_{-\pi}^{\pi} \tilde{T}_{2}^{k+1}(1) d \xi
$$

et donc

$$
\begin{aligned}
& \int_{|\xi| \geq \pi}|\xi|^{2 \sigma}|\hat{\varphi}(\xi)|^{2} d \xi \\
& \quad \leq 2^{N} \pi^{2 \sigma} \sup _{|\eta| \leq \pi}|\hat{\varphi}(\eta)|^{2} \sum_{k=0}^{\infty}\left(\frac{2^{2 \sigma}}{2^{2 N}}\right)^{k+1}\left\|\tilde{T}_{2}^{k+1}(1)\right\|_{L^{1}(-\pi, \pi)}
\end{aligned}
$$

Cette série converge dès que $\left\|\tilde{T}_{2}^{k+1}(1)\right\|_{L^{1}(-\pi, \pi)} \leq C \tau^{k+1}$ avec $\tau<$ $2^{2(N-\sigma)}$. Or on a

$$
\left\|\tilde{T}_{2}^{k+1}(1)\right\|_{L^{1}} \leq 2 \pi\left\|\tilde{T}_{2}^{k+1}(1)\right\|_{\infty} \leq 2 \pi \rho^{k+1} \max _{0 \leq r<Q} \rho^{-r}\left\|\tilde{T}_{2}^{r}(1)\right\|_{\infty}
$$

(cela provient de ce que $\left\|\tilde{T}_{2}^{r}(1)\right\|_{\infty}$ est la norme d'opérateur de $\tilde{T}_{2}^{r}$ sur $L^{\infty}(-\pi, \pi)$, et donc $\left.\left\|\tilde{T}_{2}^{r+r^{\prime}}(1)\right\|_{\infty} \leq\left\|\tilde{T}_{2}^{r}(1)\right\|_{\infty}\left\|\tilde{T}_{2}^{r^{\prime}}(1)\right\|_{\infty}\right)$. Si on a $\rho<$ $4^{N-\sigma}$, on a $\varphi \in H^{\sigma}$.

B1) implique B2). On commence par remarquer que si $\varphi \in H_{\sigma}$, pour un $\sigma>s$, alors $\varphi$ est à décroissance rapide dans $H^{t}$ pour tout $t<\sigma$ (et en particulier dans $H^{s}$ ). En fait, d'après le Lemme B, il suffit de le vérifier pour un seul $t_{0} \in \mathbb{R}$. Pour $t \leq t_{0}$, cela sera alors immédiat; pour tout $t_{0}<t<\sigma$, cela se fera par interpolation.

Puisque $|\hat{\varphi}(\xi)| \leq C(1+|\xi|)^{L}$ pour un $L \in \mathbb{N}$, on sait que la distribution

$$
\hat{\varphi}_{1}(\xi)=\left(\frac{1-e^{-i \xi}}{i \xi}\right)^{L+1} \hat{\varphi}(\xi)
$$

vérifie que $\sum_{k \in \mathbb{Z}}\left|\hat{\varphi}_{1}(\xi+2 k \pi)\right|^{2}$ converge uniformément sur $[-\pi, \pi]$, vers une fonction $\gamma_{1}$ qui ne s'annule pas sur $[-\pi, \pi]$ : si $K$ est un compact d'Albert Cohen associé à $m_{0}$, on a

$$
\inf _{\xi} \sum_{k \in \mathbb{Z}}\left|\hat{\varphi}_{1}(\xi+2 k \pi)\right|^{2} \geq \inf _{\eta \in K}\left|\frac{1-e^{-i \eta}}{i \eta}\right|^{2 L+2} \inf _{\eta \in K}|\hat{\varphi}(\eta)|^{2}>0 .
$$

On a $\hat{\varphi}_{1}(\xi)=\prod_{j=1}^{\infty} m_{1}(\xi)$ avec $m_{1}=\left(\left(1+e^{-i \xi}\right) / 2\right)^{L+1} m_{0}$. Si on définit $T_{1,2}$ par

$$
T_{1,2} f=\left|m_{1}\left(\frac{\xi}{2}\right)\right|^{2} f\left(\frac{\xi}{2}\right)+\left|m_{1}\left(\frac{\xi}{2}+\pi\right)\right|^{2} f\left(\frac{\xi}{2}+\pi\right),
$$


on a nécessairement $\left\|T_{1,2}^{N}(1)\right\|_{\infty} \leq \sup \gamma_{1} / \inf \gamma_{1}$. La Proposition 1 montre alors que $\varphi_{1}$ est à décroissance rapide dans $L^{2}$, et que $(d / d x)^{L+1} \varphi_{1}$ est à décroissance rapide dans $H^{-L-1}$. En appliquant le Lemme F, du fait que $\left(d \widehat{/ d x)^{L}}+1 \varphi_{1}=\left(1-e^{-i \xi}\right)^{L+1} \hat{\varphi}\right.$, on obtient que $\varphi$ elle-même est à décroissance rapide dans $H^{-L-1}$.

On remarque également qu'on peut supposer $s<0$. En effet, si $s \geq 0, \varphi$ est à décroissance rapide dans $L^{2}$. Cela implique que $m_{0}(\pi)=0$, comme on l'a vu dans la démonstration de la Proposition 1. Alors $m_{0}$ se factorise en $m_{0}(\xi)=\left(\left(1+e^{-i \xi}\right) / 2\right) m_{1}(\xi)$ et $\hat{\varphi}$ en $\hat{\varphi}(\xi)=\left(1-e^{-i \xi}\right) /(i \xi) \hat{\varphi}_{1}$. De plus $\varphi$ est à décroissance rapide dans $H^{s}$ si et seulement si $d \varphi / d x$ l'est dans $H^{s-1}$ (Lemme E), c'est-à-dire si et seulement si $\varphi_{1}$ l'est dans $H^{s-1}$ (Lemme F). Ainsi, si $N \leq s<N+1$, on obtient que $m_{0}(\xi)=\left(\left(1+e^{-i \xi}\right) / 2\right)^{N+1} \tilde{m}_{0}(\xi)$ et que $\varphi$ appartient à un $H^{\sigma}$ avec $\sigma>s$ si et seulement si $\tilde{\varphi}$ (définie par sa transformée de Fourier $\left.\prod_{j=1}^{\infty} \tilde{m}_{0}\left(\xi / 2^{j}\right)\right)$ appartient à un $H^{t}$ avec $t>s-N$.

On suppose donc que $s<0$ et que $\varphi \in H^{\sigma}$ pour un $\left.\sigma \in\right] s, 0[$. On a alors, si $K$ est un compact d'Albert Cohen associé à $m_{0}$

$$
\begin{aligned}
\int(1+|\xi|)^{2 \sigma}|\hat{\varphi}(\xi)|^{2} d \xi \geq & \int(1+|\xi|)^{2 \sigma} \chi_{K}\left(\frac{\xi}{2^{M}}\right)|\hat{\varphi}(\xi)|^{2} d \xi \\
\geq & \frac{1}{C}\left(2^{M}\right)^{2 \sigma} \inf _{\eta \in K}|\hat{\varphi}(\eta)|^{2} \\
& \cdot \int \chi_{K}\left(\frac{\xi}{2^{M}}\right) \prod_{j=1}^{M}\left|m_{0}\left(\frac{\xi}{2^{j}}\right)\right|^{2} d \xi
\end{aligned}
$$

D'où

$$
\int_{-\pi}^{\pi} T_{2}^{M}(1) d \xi=\int \chi_{K}\left(\frac{\xi}{2^{M}}\right) \prod_{j=1}^{M}\left|m_{0}\left(\frac{\xi}{2^{j}}\right)\right|^{2} d \xi \leq C\left(2^{2 \sigma}\right)^{-M} .
$$

On obtient que $\left\|T_{2}^{M}(1)\right\|_{L^{1}(-\pi, \pi)} \leq C \tau^{M}$, avec $\tau<4^{-s}$. (Nous avons vu qu'inversement cette inégalité assure l'appartenance de $\varphi$ à $H^{t}$ pour $\left.4^{t} \tau<1\right)$.

Nous avons obtenu une condition nécessaire et suffisante pour l'appartenance de $\varphi$ à $\cap_{\sigma>s} H^{\sigma}$ lorsque $s<0$, mais cette condition est malcommode puisqu'il s'agit de montrer que

$$
\limsup _{M \rightarrow+\infty}\left\|T_{2}^{M}(1)\right\|_{L^{1}(-\pi, \pi)}^{1 / M}<4^{-s} .
$$


Il s'agit maintenant de remplacer la norme $L^{1}$ par la norme $L^{\infty}$ de sorte à estimer un rayon spectral $\left(\left\|T_{2}^{M}(1)\right\|_{\infty}\right.$ est la norme d'opérateur de $T_{2}^{M}$ agissant sur $L^{\infty}(-\pi, \pi)$ ). Dans la littérature, c'est souvent un jeu d'enfant: on n'étudie que le cas des polynômes trigonométriques; $T_{2}$ agit alors sur un espace de dimension fini, sur lequel les normes $L^{1}$ et $L^{\infty}$ sont équivalentes. Mais nous traitons ici un cas un peu plus général $\left(m_{0} \in C^{\infty}(\mathbb{R} / 2 \pi \mathbb{Z})\right)$.

Pour contrôler la norme $\left\|T_{2}^{M}(1)\right\|_{\infty}$, il suffit de remarquer que si $\varphi \in H^{\sigma}$ pour un $\sigma>s$, alors $\varphi$ est à décroissance rapide dans $H^{t}$ pour tout $t<\sigma$ (et en particulier pour $t \in] s, \sigma[$ ); or ceci est équivalent à ce que $\varphi_{t}$, définie par $\hat{\varphi}_{t}=\left(1+\left|\xi^{2}\right|\right)^{t / 2} \hat{\varphi}$, soit à décroissance rapide dans $L^{2}$ (il suffit de vérifier que

$$
\left(\frac{d}{d \xi}\right)^{k}\left(1+|\xi|^{2}\right)^{t / 2}=\left(1+|\xi|^{2}\right)^{t / 2} \mu_{t, k}(\xi)
$$

où $\mu_{t, k}$ est $C^{\infty}$ bornée ainsi que toutes ses dérivées); le Lemme 1 donne donc

$$
\sup _{\xi \in[-\pi, \pi]} \sum_{k \in \mathbb{Z}}\left(1+|\xi+2 k \pi|^{2}\right)^{t}|\hat{\varphi}(\xi+2 k \pi)|^{2}<+\infty, \quad \text { pour } t<\sigma
$$

Soit alors $K$ un compact d'Albert Cohen associé à $m_{0}$ et $I_{M, t}$ la quantité

$$
I_{M, t}(\xi)=\sum_{k \in \mathbb{Z}} \chi_{K}\left(\frac{\xi+2 k \pi}{2^{M}}\right)\left(1+|\xi+2 k \pi|^{2}\right)^{t}|\hat{\varphi}(\xi+2 k \pi)|^{2} .
$$

Si $t<0$, on a

$$
\begin{aligned}
& \chi_{K}\left(\frac{\xi+2 k \pi}{2^{M}}\right)\left(1+|\xi+2 k \pi|^{2}\right)^{t}|\hat{\varphi}(\xi+2 k \pi)|^{2} \\
\geq & \chi_{K}\left(\frac{\frac{\xi}{2}+k \pi}{2^{M-1}}\right) 4^{t}\left(1+\left|\frac{\xi}{2}+k \pi\right|^{2}\right)^{t}\left|m_{0}\left(\frac{\xi}{2}+k \pi\right)\right|^{2}\left|\hat{\varphi}\left(\frac{\xi}{2}+k \pi\right)\right|^{2}
\end{aligned}
$$

et donc

$$
\begin{aligned}
I_{M, t} & \geq 4^{t} T_{2}\left(I_{M-1, t}\right) \\
& \geq 4^{M t} T_{2}^{M}\left(I_{0, t}\right) \\
& \geq C_{t} 4^{M t} \inf _{K}|\hat{\varphi}(\eta)|^{2} T_{2}^{M}(1)(\xi),
\end{aligned}
$$


de sorte que

$$
\left\|T_{2}^{M}(1)\right\|_{\infty} \leq C_{t}^{\prime} 4^{-M t} \sup _{\xi} \sum_{k \in \mathbb{Z}}\left(1+|\xi+2 k \pi|^{2}\right)^{t}|\hat{\varphi}(\xi+2 k \pi)|^{2} .
$$

Si on choisit $r$ et $t$ tels que $s<r<t<\sigma$, on a donc $\left\|T_{2}^{M}(1)\right\|_{\infty} \leq$ $C_{t}^{\prime \prime} 4^{-M t}$ pour tout $M$, et donc $\left\|T_{2}^{M}(1)\right\|_{\infty} \leq 4^{-M r}$ pour $M$ assez grand. Il suffit alors de poser $\rho=4^{-r}$.

Le principal argument de la preuve des propositions 1 et 2 a été la positivité de l'opérateur $T_{2}$, et l'égalité corollaire de $\left\|T_{2}\right\|_{\mathcal{L}\left(L^{\infty}, L^{\infty}\right)}$ et $\left\|T_{2}(1)\right\|_{\infty}$. Les propositions 1 et 2 s'adaptent alors aisément pour des filtres à valeurs positives:

Proposition 1 bis. Soit $M_{0} \in C^{\infty}(\mathbb{R} / 2 \pi \mathbb{Z})$ telle que

i) $M_{0}(0)=1, M_{0}(\xi) \geq 0$ et $M_{0}(\xi)=M_{0}(-\xi)$ pour tout $\xi$.

ii) $M_{0}$ vérifie le critère d'Albert Cohen.

On définit $\hat{\Phi}$ par

$$
\hat{\Phi}(\xi)=\prod_{j=1}^{\infty} M_{0}\left(\frac{\xi}{2^{j}}\right)
$$

et l'opérateur $T_{1}$ par

$$
T_{1} f(\xi)=M_{0}\left(\frac{\xi}{2}\right) f\left(\frac{\xi}{2}\right)+M_{0}\left(\frac{\xi}{2}+\pi\right) f\left(\frac{\xi}{2}+\pi\right) .
$$

Alors les assertions suivantes sont équivalentes:

C1) $\Phi$ est à décroissance rapide dans $L^{\infty}$ et pour un $\varepsilon>0$, $\Phi$ est $C^{\varepsilon}$ (régularité höldérienne)

C2) $\sup _{N \in \mathbb{N}}\left\|T_{1}^{N}(1)\right\|_{\infty}<+\infty$.

Preuve. La démonstration de la Proposition 1 s'adapte presque immédiatement.

C1) implique C2). La seule chose à vérifier est que $\sum_{k \in \mathbb{Z}} \hat{\Phi}(\xi+$ $2 k \pi$ ) converge pour tout $\xi$ vers la fonction $C^{\infty}, \sum_{k \in \mathbb{Z}} \Phi(k) e^{-i k \xi}$. Pour cela, on considère la fonction $u_{\xi}(x)=\sum_{k \in \mathbb{Z}} \Phi(x+k) e^{-i(x+k) \xi}$. 
C'est une fonction périodique, bornée et höldérienne: si $\omega$ est $C^{\varepsilon}$ et à décroissance rapide dans $L^{\infty}$, on a

$$
\begin{gathered}
\left|\sum_{k \in \mathbb{Z}} \omega(x+k)\right| \leq\left\|\omega(x)\left(1+x^{2}\right)\right\|_{\infty} \sum_{k \in \mathbb{Z}} \frac{1}{1+(x+k)^{2}} \leq C<+\infty, \\
\left|\sum_{k \in \mathbb{Z}} \omega(x+h+k)-\omega(x+k)\right| \\
\leq\|\omega\|_{C^{\varepsilon}}|h|^{\varepsilon}\left(\sum_{|x+k| \leq K_{0}} 1\right)+2\left\|\omega(x) x^{2}\right\|_{\infty} \sum_{|x+k|>K_{0}} \frac{1}{|x+k|^{2}} \\
\leq C\left(K_{0}|h|^{\varepsilon}+\frac{1}{K_{0}}\right) \leq C^{\prime}|h|^{\varepsilon / 2},
\end{gathered}
$$

pour $|h| \leq 1$ et $K_{0} \approx|h|^{-\varepsilon / 2}$.

Puisque $u_{\xi}$ est höldérienne, on a

$$
\sum_{k \in \mathbb{Z}} \Phi(x+k) e^{-i(x+k) \xi}=\sum_{k \in \mathbb{Z}} \hat{\Phi}(\xi+2 k \pi) e^{+2 i k \pi x}
$$

pour tout $x$; en prenant $x=0$, on obtient la convergence souhaitée.

$\mathrm{C} 2$ ) implique $\mathrm{C} 1$ ). La démonstration est exactement similaire à celle de la Proposition 1 . On obtient en particulier que pour tout $p \in \mathbb{N}$,

$$
\sum_{k \in \mathbb{Z}}\left|\frac{d^{p}}{d \xi^{p}} \hat{\Phi}(\xi+2 k \pi)\right|<+\infty
$$

ce qui entraîne $x^{p} \Phi \in C_{0}$.

Proposition 2 bis. Soit $M_{0} \in C^{\infty}(\mathbb{R} / 2 \pi \mathbb{Z})$ telle que

i) $M_{0}(0)=1, M_{0}(\xi) \geq 0$ et $M_{0}(\xi)=M_{0}(-\xi)$ pour tout $\xi \in \mathbb{R}$.

ii) $M_{0}$ vérifie le critère d'Albert Cohen.

iii) $\sup _{N \in \mathbb{N}}\left\|T_{1}^{N}(1)\right\|_{\infty}<+\infty$ ò̀

$$
T_{1} f=M_{0}\left(\frac{\xi}{2}\right) f\left(\frac{\xi}{2}\right)+M_{0}\left(\frac{\xi}{2}+\pi\right) f\left(\frac{\xi}{2}+\pi\right) .
$$

Soit $\hat{\Phi}=\prod_{j=1}^{\infty} M_{0}\left(\xi / 2^{j}\right)$. Alors les assertions suivantes sont équivalentes, pour $\alpha \geq 0$. 
D1) Il existe $\beta>\alpha$ tel que $|\xi|^{\beta} \hat{\Phi} \in L^{1}$ (ce qui équivaut à $\Phi \in B_{1}^{\beta, \infty}$ puisque $\hat{\Phi}$ est positive ou nulle).

D2) En écrivant $\alpha=2 N+\theta, N \in \mathbb{N}, 0 \leq \theta<2, M_{0}$ vérifie les conditions suivantes:

i) $M_{0}=((1+\cos \xi) / 2)^{N+1} \tilde{M}_{0}$ avec $\tilde{M}_{0} \in C^{\infty}(\mathbb{R} / 2 \pi \mathbb{Z})$.

ii) Il existe $\rho \in\left[1,2^{2-\theta}\left[\right.\right.$ et $Q \in \mathbb{N}^{*}$ tels que

$$
\left\|\tilde{T}_{1}^{Q}(1)\right\|_{\infty} \leq \rho^{Q}
$$

où

$$
\tilde{T}_{1} f(\xi)=\tilde{M}_{0}\left(\frac{\xi}{2}\right) f\left(\frac{\xi}{2}\right)+\tilde{M}_{0}\left(\frac{\xi}{2}+\pi\right) f\left(\frac{\xi}{2}+\pi\right)
$$

Preuve. D2) implique D1). Comme pour la Proposition 2.

D1) implique D2). On commence par remarquer que si $\Phi \in B_{1}^{\beta, \infty}$, alors $\Phi$ est à décroissance rapide dans $B_{1}^{\gamma, \infty}$ pour tout $\gamma<\beta$ (on utilise l'inclusion $L^{\infty} \subset B_{1}^{\delta, \infty}$ pour tout $\delta<0$ et le lemme B pour $B_{1}^{\delta, \infty}$ et $\left.B_{1}^{\beta, \infty}\right)$.

On montre alors que $\Phi_{1}$, de transformée de Fourier $\prod_{j=1}^{\infty} \tilde{M}_{0}\left(\xi / 2^{j}\right)$, est à décroissance rapide dans $B_{1}^{\gamma-2 N-2, \infty}$ pour tout $\gamma<\beta$ (en particulier pour $\alpha<\gamma<\beta$ ). On obtient alors que

$$
\int_{-\pi}^{\pi} \tilde{T}_{1}^{M}(1) d \xi \leq C 2^{(2 N+2-\gamma) M}
$$

pour tout $M$ et le problème est à nouveau de passer de la norme $L^{1}$ à la norme $L^{\infty}$. Pour cela, on note $u_{\gamma}$ la fonction définie par

$$
\hat{u}_{\gamma}=|\xi|^{2 N+2}\left(1+|\xi|^{2}\right)^{(\gamma-2 N-2) / 2} \hat{\Phi} .
$$

On a $(1+|\xi|)^{\beta-\gamma} \hat{u}_{\gamma} \in L^{1}$, de sorte que $u_{\gamma} \in B_{1}^{\beta-\gamma, \infty}$. De plus, $\Phi$ est à décroissance rapide dans $L^{\infty}$, donc dans $B_{1}^{\delta, \infty}$ pour tout $\delta<0$; on en conclut que $u_{\gamma}$ est à décroissance rapide dans $B_{1}^{\delta-\gamma, \infty}$ (il suffit de vérifier que $\left(d^{2} / d \xi^{2}\right)^{N+1} u_{\gamma}$ est à décroissance rapide dans $B_{1}^{\delta-2 N-2, \infty}$, puisque pour tout $\rho \in \mathbb{R}$ et $\sigma \in \mathbb{R},(\operatorname{Id}-\Delta)^{\rho}$ envoie $B_{1}^{\sigma, \infty}$ dans $B_{1}^{\sigma-\rho, \infty}$ tandis que $\left[x,(\operatorname{Id}-\Delta)^{\rho}\right]=(\operatorname{Id}-\Delta)^{\rho} \circ S_{\rho}$ où $S_{\rho}$ envoie $B_{1}^{\sigma, \infty}$ dans $\left.B_{1}^{\sigma, \infty}\right)$. 
On en conclut que $u_{\gamma}$ est à décroissance rapide dans $B_{1}^{\sigma, \infty}$ pour tout $\sigma<\beta-\gamma$, donc (puisque $\gamma<\beta$ ) dans $L^{\infty}$. On sait alors que

$$
\begin{aligned}
\sum_{k \in \mathbb{Z}}(1+|\xi+2 k \pi|)^{(\gamma-2 N-2) / 2}|\xi+2 k \pi|^{2 N+2} \hat{\Phi}(\xi+2 k \pi) & \\
& =\sum_{k \in \mathbb{Z}} u_{\gamma}(k) e^{-i k \xi} \in C^{\infty}
\end{aligned}
$$

De plus, on a

$$
\sum_{k \in \mathbb{Z}}(x-k)^{p} u_{\gamma}(x-k)=\sum_{k \in \mathbb{Z}}\left(i \frac{\partial}{\partial \xi}\right)^{p} \hat{u}_{\gamma}(2 k \pi) e^{2 i k \pi x}
$$

au sens des distributions (la première série convergeant uniformément sur tout compact); pour $0 \leq p \leq N+1$, on obtient

$$
\sum_{k \in \mathbb{Z}}(x-k)^{p} u_{\gamma}(x-k)=0
$$

D'où

$$
\left.\left(\frac{d}{d \xi}\right)^{p} \sum_{k \in \mathbb{Z}} u_{\gamma}(k) e^{-i k \xi}\right|_{\xi=0}=\sum_{k \in \mathbb{Z}}(-i k)^{p} u_{\gamma}(k)=0
$$

et donc

$$
\sum_{k \in \mathbb{Z}} u_{\gamma}(k) e^{-i k \xi}=(1-\cos \xi)^{N+1} M(\xi), \quad \text { avec } M \in C^{\infty}(\mathbb{R} / 2 \pi \mathbb{Z})
$$

Cela donne

$$
\sum_{k \in \mathbb{Z}}(1+|\xi+2 k \pi|)^{(\gamma-2 N-2) / 2)} \hat{\Phi}_{1}(\xi+2 k \pi) \in C^{\infty}(\mathbb{R} / 2 \pi \mathbb{Z})
$$

$\left(\operatorname{car} \hat{\Phi}(\xi)=\left(\xi^{2} /(2(1-\cos \xi))^{N+1} \hat{\Phi}_{1}(\xi)\right)\right.$ et la démonstration peut alors se faire.

Enfin, le dernier résultat classique caractérise les fonctions d'échelle interpolantes et les fonctions d'échelle orthonormées: 
Proposition 3. Soit $m_{0} \in C^{\infty}(\mathbb{R} / 2 \pi \mathbb{Z})$ telle que $m_{0}(0)=1, m_{0}(\xi)=$ $\overline{m_{0}(-\xi)}$ et $m_{0}$ satisfait le critère d'Albert Cohen, et soit

$$
\hat{\varphi}=\prod_{j=1}^{\infty} m_{0}\left(\frac{\xi}{2^{j}}\right)
$$

i) Les deux assertions suivantes sont équivalentes:

E1) La famille $\{\varphi(x-k)\}_{k \in \mathbb{Z}}$ est orthonormée.

E2) $\left|m_{0}(\xi)\right|^{2}+\left|m_{0}(\xi+\pi)\right|^{2}=1$, pour tout $\xi \in \mathbb{R}$.

ii) Si $m_{0}(\xi) \geq 0$ pour tout $\xi$, alors les deux assertions suivantes sont équivalentes:

F1) $\varphi \in C_{0}, \varphi(0)=1$ et pour $k \in \mathbb{Z}, k \neq 0, \varphi(k)=0$.

F2) $m_{0}(\xi)+m_{0}(\xi+\pi)=1$, pour tout $\xi \in \mathbb{R}$.

Preuve. Il suffit de démontrer l'équivalence entre F1) et F2). En effet, la famille $\{\varphi(x-k)\}_{k \in \mathbb{Z}}$ est orthonormée si et seulement si la fonction $\Phi(x)=\int \varphi(y) \varphi(y-x) d y$ vérifie $\Phi(0)=1$ et $\Phi(k)=0$; or on a $\hat{\Phi}(\xi)=\prod_{j=1}^{\infty}\left|m_{0}\left(\xi / 2^{j}\right)\right|^{2}$.

Maintenant si $m_{0}(\xi) \geq 0$ et si $\varphi$ est bornée, alors $\hat{\varphi}$ doit être intégrable de sorte que $\sum_{k \in \mathbb{Z}} \hat{\varphi}(\xi+2 k \pi)$ converge presque partout. De plus, au sens des distributions

$$
\sum_{k \in \mathbb{Z}} \hat{\varphi}(\xi+2 k \pi)=\sum_{k \in \mathbb{Z}} \varphi(k) e^{-i k \xi}
$$

Si $\varphi$ vérifie $\mathrm{F} 1)$, on trouve $\sum_{k \in \mathbb{Z}} \hat{\varphi}(\xi+2 k \pi)=1$ presque pour tout, ce qui entraîne F2) presque partout (et donc partout).

Inversement si $m_{0}$ vérifie $\mathrm{F} 2$ ), alors $T_{1}(1)=1$ et donc $\hat{\varphi} \in L^{1}$ d'après la Proposition 1 bis. On sait alors que si $K$ est un compact d'Albert Cohen,

$$
\hat{\theta}_{M}=\prod_{j=1}^{M} m_{0}\left(\frac{\xi}{2^{j}}\right) \chi_{K}\left(\frac{\xi}{2^{M}}\right) \longrightarrow \hat{\varphi}
$$

dans $L^{1}$, et donc $\theta_{M} \rightarrow \varphi$ dans $L^{\infty}$. Mais il est facile de vérifier que $\theta(0)=1$ et $\theta(k)=0$ pour $k \in \mathbb{Z}^{*}$ : cela se déduit par récurrence sur $M$ à partir de $\sum_{k \in \mathbb{Z}} \chi_{K}(\xi+2 k \pi)=1$ presque pour tout et donc $\theta_{0}(0)=1$, $\theta_{0}(k)=0$ pour $k \neq 0$. 
Nous avons maintenant tous les outils de la théorie "classique" (légèrement remaniée pour les besoins de la cause) dont nous aurons besoin par la suite.

\section{Dépendance de la fonction d'échelle par rapport à son filtre.}

Nous pouvons maintenant facilement aborder le problème de la dépendance de la fonction d'échelle $\varphi$ par rapport à son filtre $m_{0}$. C'est-à-dire que peut-on dire des fonctions d'échelle $\varphi_{n}$ dont les filtres d'échelle $m_{n}$ convergent dans $C^{\infty}(\mathbb{R} / 2 \pi \mathbb{Z})$ vers une fonction $m_{\infty}$ ? En fait, la réponse est étonnamment simple. La convergence est simple à vérifier; pour la convergence des dérivées, il faut que celles-ci existent, et donc au moins que les filtres $m_{n}$ s'annulent suffisamment en $\pi$ (et en fait ce sera la seule restriction!).

Théoréme 1 (Premier Théorème d'approximation). Soit $m_{\infty} \in C^{\infty}$ $(\mathbb{R} / 2 \pi \mathbb{Z})$ vérifiant le critère d'Albert Cohen et soit

$$
\left\{m_{n}\right\}_{n \in \mathbb{N}} \in C^{\infty}(\mathbb{R} / 2 \pi \mathbb{Z})^{\mathbb{N}}
$$

une suite de fonctions qui convergent vers $m_{\infty}$ dans $C^{\infty} \underline{(\mathbb{R} / 2 \pi \mathbb{Z})}$. On suppose que pour tout $n \in \mathbb{N}, m_{n}(0)=1$ et $m_{n}(\xi)=\overline{m_{n}(-\xi)}$. On définit $\varphi_{n}(n \in \mathbb{N} \cup\{\infty\})$ par

$$
\hat{\varphi}_{n}=\prod_{j=1}^{\infty} m_{n}\left(\frac{\xi}{2^{j}}\right) .
$$

i) Soit $\alpha \in \mathbb{R}$. Alors les assertions suivantes sont équivalentes:

G1) Pour un $\beta>\alpha, \varphi_{n}$ est à décroissance rapide dans $H^{\beta}$ pour $n$ suffisamment grand et $\varphi_{n}$ converge rapidement vers $\varphi_{\infty}$ dans $H^{\beta}$.

G2) Pour un $\gamma>\alpha, \varphi_{\infty} \in H^{\gamma}$ et pour $n$ suffisamment grand on a, pour $0 \leq p \leq N_{\alpha},(d / d \xi)^{p} m_{n}(\pi)=0$ où $N_{\alpha}=-1$ si $\alpha<0$ et $N_{\alpha}=[\alpha]$ si $\alpha \geq 0$.

ii) De même si les $m_{N}$ sont toutes à valeurs positives ou nulles, et si $\alpha \geq 0$ alors les assertions suivantes sont équivalentes:

H1) Pour un $\beta>\alpha, \varphi_{n}$ est à décroissance rapide dans $B_{1}^{\beta, \infty}$ pour $n$ suffisamment grand et $\varphi_{n}$ converge rapidement vers $\varphi_{\infty}$ dans $B_{1}^{\beta, \infty}$. 
H2) Pour un $\gamma>\alpha, \varphi_{\infty} \in B_{1}^{\gamma, \infty}$ et pour $n$ suffisamment grand, on a, pour $0 \leq p \leq 2 N_{\alpha}+1,\left(d^{p} / d \xi^{p}\right) m_{n}(\pi)=0$ où $N_{\alpha}=[\alpha / 2]$.

REMARQue. On peut bien sûr remplacer l'espace de Besov $B_{1}^{\beta, \infty}$ par l'espace plus naturel $B_{\infty}^{\beta, \infty}$ (puisque pour $\beta_{1}<\beta_{2}, B_{1}^{\beta_{2}, \infty} \subset B_{\infty}^{\beta_{2}, \infty} \subset$ $\left.B_{1}^{\beta_{1}, \infty}\right)$ : si $\beta>0$ et $\beta \notin \mathbb{N}, B_{\infty}^{\beta, \infty}$ est l'espace des fonctions de classe $C^{[\beta]}$, bornées ainsi que leurs dérivées jusqu'à l'ordre $[\beta]$ et telles que leur dérivée $[\beta]$-ième soit höldérienne d'exposant $\beta-[\beta]$.

Preuve. On commence par remarquer qu'on peut supposer sans perte de généralité que les filtres $\left\{m_{n}\right\}_{n \in \mathbb{N} \cup\{\infty\}}$ vérifient tous le critère d'Albert Cohen pour un même compact $K$. En effet, $\hat{\varphi}_{n} \rightarrow \hat{\varphi}_{\infty}$ uniformément sur tout compact: sur un voisinage $V$ de 0 , il suffit de passer au logarithme pour le voir; le cas général s'en déduit aisément en écrivant pour $\xi \in 2^{L} V$,

$$
\varphi_{n}(\xi)=\hat{\varphi}_{n}\left(\frac{\xi}{2^{L}}\right) \prod_{j=1}^{L} m_{n}\left(\frac{\xi}{2^{j}}\right) .
$$

Si $K$ est un compact d'Albert Cohen associé à $m_{\infty}$, on $\operatorname{ainf}_{K}\left|\hat{\varphi}_{\infty}(\xi)\right|>$ 0 ; on obtient alors que $\inf _{K}\left|\hat{\varphi}_{n}(\xi)\right|>0$ pour $n$ assez grand.

Les implications G1) implique G2) et H1) implique H2) sont immédiates: d'après les propositions 2 et 2 bis, si $\varphi_{n} \in H^{\beta}$ pour un $\beta>\alpha$, alors $m_{n}$ peut se factoriser en $\left(\left(1+e^{-i \xi}\right) / 2\right)^{N_{\alpha}+1} \tilde{m}_{n}(\xi)$ et de même on a un résultat analogue si $\varphi_{n} \in B_{1}^{\beta, \infty}$.

G2) implique G1). On factorise $m_{n}$ en $\left(\left(1+e^{-i \xi}\right) / 2\right)^{N_{\alpha}+1} \tilde{m}_{n}(\xi)$ pour $n \geq n_{0}$ (où $n \geq n_{0}$ implique $\left(d^{p} / d \xi^{p}\right) m_{n}(\pi)=0$ pour $0 \leq p \leq$ $\left.N_{\alpha}\right)$. Alors $\tilde{m}_{n} \in C^{\infty}(\mathbb{R} / 2 \pi \mathbb{Z})$ et converge dans $C^{\infty}$ vers $\tilde{m}_{\infty}$. On pose

$$
\tilde{\omega}_{n}=\prod_{j=1}^{\infty} \tilde{m}_{n}\left(\frac{\xi}{2^{j}}\right)
$$

et

$$
\tilde{T}_{2, n} f=\left|\tilde{m}_{n}\left(\frac{\xi}{2}\right)\right|^{2} f\left(\frac{\xi}{2}\right)+\left|\tilde{m}_{n}\left(\frac{\xi}{2}+\pi\right)\right|^{2} f\left(\frac{\xi}{2}+\pi\right) .
$$

Puisque $\varphi_{\infty} \in H^{\beta}$, on sait qu'il existe $\left.\gamma \in\right] \alpha, \beta\left[\right.$ et $Q \in \mathbb{N}^{*}$ tel que

$$
\left\|\tilde{T}_{2, \infty}^{Q}(1)\right\|_{\infty} \leq 4^{\left(N_{\alpha}+1-\gamma\right) Q} .
$$

Comme $\tilde{T}_{2, n}^{Q}(1) \rightarrow \tilde{T}_{2, \infty}^{Q}(1)$ dans $C^{\infty}(\mathbb{R} / 2 \pi \mathbb{Z})$, on conclut que si $\delta \in$ ] $\alpha, \gamma\left[\right.$ il existe $n_{1}$ tel que pour $n \geq n_{1}$

$$
\left\|\tilde{T}_{2, n}^{Q}(1)\right\|_{\infty} \leq 4^{\left(N_{\alpha}+1-\delta\right) Q}
$$


et enfin

$$
\sup _{n \geq n_{1}}\left\|\varphi_{n}\right\|_{H^{\sigma}}<+\infty, \quad \text { pour tout } \sigma<\delta .
$$

Par ailleurs, on vérifie facilement qu'il existe $B \in \mathbb{R}$ tel que

$$
\sup _{n}\left\|(1+|\xi|)^{B} \hat{\varphi}_{n}\right\|_{\infty}<+\infty .
$$

(Bien sûr, $B$ est très négatif). La convergence ponctuelle de $\hat{\varphi}_{n}$ vers $\hat{\varphi}_{\infty}$ donne que $\varphi_{n}$ converge vers $\varphi_{\infty}$ dans $H^{\sigma}$ pour tout $\sigma<B-1 / 2$. On obtient donc que $\varphi_{n}$ converge vers $\varphi_{\infty}$ dans $H^{\sigma}$ pour tout $\sigma<\delta$ (par interpolation entre $H^{B-1}$ et $H^{(\sigma+\delta) / 2}$ ).

Par ailleurs

$$
\left(\frac{1-e^{-i \xi}}{i \xi}\right)^{M} \hat{\varphi}_{n}=\hat{\omega}_{n}
$$

avec $M \in \mathbb{N}, M>-B+1 / 2$, vérifie

$$
\sum_{k \in \mathbb{Z}}\left|\hat{\omega}_{n}(\xi+2 k \pi)\right|^{2}<+\infty
$$

pour $n \geq n_{0}$, et donc $\left(\left(1+e^{-i \xi}\right) / 2\right)^{M} m_{n}$ dans $L^{2}$. De plus, $\left\|x^{k} \omega_{n}\right\|_{2}$ se majore à l'aide des quantités $\left\|\mu_{n}^{(p)}\right\|_{\infty}$ (où $\mu_{n}=\left(\left(1+e^{-i \xi}\right) / 2\right)^{M} m_{n}$ ) pour $0 \leq p \leq k$ et $\sup _{N \in \mathbb{N}}\left\|T_{2, n}^{N}(1)\right\|_{\infty}$ et $\rho_{0, n}<1$ tel que pour un $Q$,

$$
T_{2, n}^{Q}\left(\left|\sin \frac{\xi}{2}\right|\right) \leq \rho_{0, n}^{Q}\left|\sin \frac{\xi}{2}\right|
$$

oú

$$
T_{2, n}(f)=\left|\mu_{n}\left(\frac{\xi}{2}\right)\right|^{2} f\left(\frac{\xi}{2}\right)+\left|\mu_{n}\left(\frac{\xi}{2}+\pi\right)\right|^{2} f\left(\frac{\xi}{2}+\pi\right) .
$$

Or on a bien évidemment: $\sup _{n}\left\|\mu_{n}^{(p)}\right\|_{\infty}<+\infty$; par ailleurs, on a

$$
\sup _{n \geq n_{0}} \sup _{N}\left\|T_{2, n}^{N}(1)\right\|_{\infty} \leq \sup _{n \geq n_{0}} \frac{\sup _{\xi \in[-\pi, \pi]} \sum_{k \in \mathbb{Z}}\left|\hat{\omega}_{n}(\xi+2 k \pi)\right|^{2}}{\inf _{\xi \in K}\left|\hat{\omega}_{n}(\xi)\right|^{2}}<+\infty,
$$

où $K$ est un compact d'Albert Cohen commun à tous les $m_{n}$. Enfin, on écrit $\mu_{n}=\left(\left(1+e^{-i \xi}\right) / 2\right) \tilde{\mu}_{n}$ et

$$
T_{2, n}\left(\left|\sin \frac{\xi}{2}\right| f\right)=\frac{1}{2}\left|\sin \frac{\xi}{2}\right| S_{2, n}(f),
$$


ou

$$
S_{2, n}(f)=\left|\cos \frac{\xi}{2}\right|\left|\tilde{\mu}_{n}\left(\frac{\xi}{2}\right)\right|^{2} f\left(\frac{\xi}{2}\right)+\left|\sin \frac{\xi}{2}\right|\left|\tilde{\mu}_{n}\left(\frac{\xi}{2}+\pi\right)\right|^{2} f\left(\frac{\xi}{2}+\pi\right) .
$$

On a

$$
T_{2, \infty}^{Q}\left(\left|\sin \frac{\xi}{2}\right|\right) \leq \lambda\left|\sin \frac{\xi}{2}\right|
$$

avec $\lambda<1$ si et seulement si $S_{2, \infty}^{Q}(1) \leq \lambda$ avec $\lambda<2^{Q}$. Or $S_{2, n}^{Q}(1)$ converge uniformément vers $S_{2, \infty}^{Q}$. Si on choisit $\left.\lambda^{\prime} \in\right] \lambda, 2^{Q}[$, on trouve que $\left\|S_{2, n}^{Q}(1)\right\|_{\infty} \leq \lambda^{\prime}$ pour $n$ assez grand, et donc

$$
T_{2, n}^{Q}\left(\left|\sin \frac{\xi}{2}\right|\right) \leq \rho_{0}^{Q}\left|\sin \frac{\xi}{2}\right|
$$

avec $\rho_{0}=\left(2^{-Q} \lambda^{\prime}\right)^{1 / Q}$. On en conclut que, pour tout $p \in \mathbb{N}$,

$$
\sup _{n \geq n_{1}}\left\|x^{p} \omega_{n}\right\|_{2}<+\infty
$$

et donc $\sup _{n \geq n_{1}}\left\|x^{p} \varphi_{n}\right\|_{H^{-M}}<+\infty$. Par interpolation, $\varphi_{n}$ est à décroissance rapide et converge rapidement vers $\varphi_{\infty}$ dans $H^{\sigma}$ pour tout $\sigma<\delta$.

H2) implique H1). La démonstration est exactement similaire à G2) implique G1), mutatis mutandis.

Ce premier théorème d'approximation est donc une adaptation assez directe de la théorie "classique" des fonctions d'échelle. Mais sa méthodologie peut facilement s'étendre à des fonctions d'échelle non classiques: les fonctions d'échelle non stationnaires.

\section{Analyse multi-résolution non-stationnaire.}

Nous allons donner ici une définition très personnelle des analyses multi-résolutions non-stationnaires, et nous appellerons "analyse multirésolution non-stationnaire normale" ce que d'autres auteurs appellent simplement "analyse multi-résolution non-stationnaire".

Definition 5. Soit $\mu \in L_{\mathbb{R}}^{2}(\mathbb{R})$ une fonction à valeurs réelles et $\grave{a}$ support compact $\mu \neq 0$. L'analyse multi-résolution non stationnaire 
associée à $\mu$ est la famille des sous-espaces $\left\{V_{j}[\mu]\right\}_{j \in \mathbb{N}}$ de $L^{2}(\mathbb{R})$ définis par $f \in V_{j}[\mu]$ si et seulement si $f \in L^{2}$ et il existe $\left\{a_{k}\right\} \in \mathbb{C}^{\mathbb{Z}}$ tel que

$$
f=\sum_{k} a_{k} \mu\left(x-\frac{k}{2^{j}}\right) \quad \text { dans } \mathcal{D}^{\prime} .
$$

Bien sûr, $f \in V_{j}[\mu]$ si et seulement si $f\left(x / 2^{j}\right) \in V_{0}\left[\mu\left(\cdot / 2^{j}\right)\right]$. Dans un premier temps, nous commençons donc par décrire les propriétés de $V_{0}[\mu]$. Nous écrivons $L_{\text {comp }}^{2}=\left\{f \in L_{\mathbb{R}}^{2}: \operatorname{supp} f\right.$ est compact $\}$.

\section{Lemme 6.}

i) Il existe $\varphi_{0} \in V_{0}[\mu] \cap L_{\text {comp }}^{2}, \omega_{0} \in L_{\text {comp }}^{2}$ tel que

$$
\left\langle\varphi_{0}(x), \omega_{0}(x-k)\right\rangle=\delta_{k, 0}, \quad \text { pour tout } k \in \mathbb{Z} .
$$

ii) Pour $f \in V_{0}[\mu]$,

$$
f(x)=\sum_{k}\left\langle f, \omega_{0}(x-k)\right\rangle \varphi_{0}(x-k) .
$$

iii) $V_{0}[\mu]$ est le plus petit sous-espace fermé de $L^{2}(\mathbb{R})$ contenant $\mu$ et ses translatées $\mu(x-k), k \in \mathbb{Z}$, et la famille $\left\{\varphi_{0}(x-k)\right\}_{k \in \mathbb{Z}}$ est une base de Riesz de $V_{0}[\mu]$.

iv) Si $\tilde{\varphi}_{0}, \tilde{\omega}_{0}$ vérifient les mêmes propriétés que $\varphi_{0}, \omega_{0}$ alors, il existe $\lambda \neq 0, k \in \mathbb{Z}$ telle que $\tilde{\varphi}_{0}(x)=\lambda \varphi_{0}(x-k)$.

En fait $\varphi_{0}$ peut être définie comme la fonction de $V_{0}[\mu]$ de support minimum. Si $h \in L_{\text {comp }}^{2}$, on note $\langle h\rangle$ le nombre

$$
\langle h\rangle=\sup \operatorname{supp} h-\inf \operatorname{supp} h \text {. }
$$

On a alors

$$
\left\langle\varphi_{0}\right\rangle=\min \left\{\langle\varphi\rangle: \varphi \in V_{0}[\mu] \cap L_{\text {comp }}^{2}, \varphi \neq 0\right\} .
$$

C'est évident: si $\varphi \in L_{\text {comp }}^{2}$, on applique le point ii) du Lemme 6 pour voir que $\varphi$ est une combinaison linéaire finie des $\varphi_{0}(x-k): \varphi(x)=$ $\sum_{k_{0}}^{k_{1}} a_{k} \varphi_{0}(x-k), a_{k_{0}} \neq 0, a_{k_{1}} \neq 0($ si $\varphi \neq 0)$; on a alors $\langle\varphi\rangle=$ $\left\langle\varphi_{0}\right\rangle+k_{1}-k_{0}$. En particulier, le point iv) est évident: $\left\langle\tilde{\varphi}_{0}\right\rangle=\left\langle\varphi_{0}\right\rangle$, et donc $k_{1}\left(\tilde{\varphi}_{0}\right)=k_{0}\left(\tilde{\varphi}_{0}\right)$ de sorte que $\tilde{\varphi}_{0}=a_{k_{0}} \varphi_{0}\left(x-k_{0}\right)$. 
Preuve du Lemme 6 . Notons $X_{0}$ le plus petit sous-espace fermé de $L^{2}$ qui contienne toutes les fonctions $\mu(x-k), k \in \mathbb{Z}$. Pour $h, g \in L_{\text {comp }}^{2}$, notons

$$
P_{h, g}(\xi)=\sum_{k \in \mathbb{Z}}\langle h(x), g(x-k)\rangle e^{-i k \xi} .
$$

Alors l'ensemble $I_{g}=\left\{P_{h, g}: h \in C_{c}^{\infty}\right\}$ est un idéal de l'anneau des polynômes trigonométriques: $P_{h_{1}, g}+P_{h_{2}, g}=P_{h_{1}+h_{2}, g}$ et $e^{-i k \xi} P_{h, g}=$ $P_{h(x-k), g}$. Si $g \neq 0, I_{g}$ a un unique générateur $P_{g}=1+\sum_{k \geq 1} \alpha_{k} e^{-i k \xi}$ (où la suite $\alpha_{k}$ est presque nulle). On note $d(g)=\operatorname{deg} P_{g}$. On peut alors choisir $\varphi_{0} \in X_{0} \cap L_{\text {comp }}^{2}$ et $\omega_{0} \in C_{c}^{\infty}$ tels que

$$
\left\{\begin{array}{l}
d\left(\varphi_{0}\right)=\min \left\{d(\varphi) / \varphi \in L_{\text {comp }}^{2} \cap X_{0}: \varphi \neq 0\right\}, \\
P_{\omega_{0}, \varphi_{0}}=P_{\varphi_{0}} .
\end{array}\right.
$$

Pour démontrer i), il suffit de montrer que $P_{\varphi_{0}}=1$, ou encore que

$$
P_{\varphi_{0}}(z)=\sum_{k \in \mathbb{Z}}\left\langle\omega_{0}(x), \varphi_{0}(x-k)\right\rangle z^{k}
$$

ne s'annule jamais. Mais cela est (presque) évident. Si $P_{\varphi_{0}}\left(\bar{z}_{0}\right)=0$, alors $P_{h, \varphi_{0}}\left(\bar{z}_{0}\right)=0$ pour tout $h \in C_{c}^{\infty}$, de sorte que

$$
\sum_{k \in \mathbb{Z}} z_{0}^{k} \varphi_{0}(x-k)=0
$$

dans $\mathcal{D}^{\prime}$. On pose alors

$$
\tilde{\varphi}_{0}=\sum_{k \geq 0} z_{0}^{k} \varphi_{0}(x-k)=-\sum_{k<0} z_{0}^{k} \varphi_{0}(x-k) .
$$

On a $\varphi(x)=\tilde{\varphi}_{0}(x)-z_{0} \tilde{\varphi}_{0}(x-1)$, de sorte que

$$
P_{\varphi_{0}}=P_{\omega_{0}, \varphi_{0}}=\left(1-z_{0} e^{i x \xi}\right) P_{\omega_{0}, \tilde{\varphi}_{0}},
$$

ce qui contredit la définition de $\varphi_{0}$ si jamais $\tilde{\varphi}_{0} \in X_{0} \cap L_{\text {comp }}^{2}$. Mais bien évidemment $\tilde{\varphi}_{0}$ est à support compact (car le support de $\sum_{k \geq 0} z_{0}^{k} \varphi_{0}(x-$ $k$ ) est contenu dans [inf supp $\varphi_{0},+\infty\left[\right.$ et celui de $\sum_{k<0} z_{0}^{k} \varphi_{0}(x-k)$ dans ]$\left.\left.-\infty,-1+\sup \operatorname{supp} \varphi_{0}\right]\right)$. Par ailleurs, si $\left|z_{0}\right|<1, \sum_{k \geq 0} z_{0}^{k} \varphi_{0}(x-k)$ converge dans $L^{2}$ et $\tilde{\varphi}_{0} \in X_{0}$; si $\left|z_{0}\right|>1, \sum_{k<0} z_{0}^{k} \varphi_{0}(x-k) \in X_{0}$. Si $\left|z_{0}\right|=1$, on a

$$
\left\|\sum_{k=0}^{N} z_{0}^{k} \varphi_{0}(x-k)\right\|_{2}=\left\|\tilde{\varphi}_{0}-z_{0}^{N+1} \tilde{\varphi}_{0}(x-N-1)\right\|_{2} \leq 2\left\|\tilde{\varphi}_{0}\right\|_{2}
$$


$\left(\tilde{\varphi}_{0} \in L^{2}\right.$, puisque $\tilde{\varphi}_{0} \in L_{\text {loc }}^{2}$ et $\tilde{\varphi}_{0}$ a un support compact); par ailleurs, $\tilde{\varphi}_{0}-z_{0}^{N+1} \tilde{\varphi}_{0}(x-N-1)$ converge vers $\tilde{\varphi}_{0}$ dans $\mathcal{D}^{\prime}$, et donc faiblement dans $L^{2}$, de sorte que $\tilde{\varphi}_{0}$ appartient à l'adhérence faible du convexe fermé $X_{0}$, donc à $X_{0}$. Le point i) est donc démontré. Pour le point ii), il suffit de remarquer que si $P_{\mu}=C \prod_{1}^{K}\left(X-z_{k}\right)$, alors

$$
\hat{\mu}(\xi)=C^{\prime} e^{i N \xi} \prod\left(e^{-i \xi}-z_{k}\right) \hat{\varphi}_{0}(\xi)
$$

En effet, la technique d'expulsion des zéros que nous avons utilisée entre $\varphi_{0}$ et $\tilde{\varphi}_{0}$ dans la preuve du point i) peut s'appliquer à $\hat{\mu}$ jusqu'à complète élimination. Nous obtenons alors une fonction $\varphi_{0}$ telle que $P_{\varphi_{0}}=1$ et telle que $\mu$ s'exprime comme une combinaison linéaire finie des $\varphi_{0}(x-k)$. La famille $\varphi_{0}(x-k)$ est une base de Riesz de $X_{0}$ et pour cette fonction $\varphi_{0}$ au moins la propriété ii) est évidente. Il suffit alors de remarquer que si $\varphi \in X_{0} \cap L_{\text {comp }}^{2}$ alors $d(\varphi)>d\left(\varphi_{0}\right)$ sauf si $\varphi=\lambda \varphi_{0}(x-k)$, ce qui se vérifie en décomposant $\varphi$ sur les $\varphi_{0}(x-k)$.

Le seul point qui reste à vérifier est $X_{0}=V_{0}[\mu]$. On remarque d'abord que $X_{0}^{\perp} \cap L_{\text {comp }}^{2}$ est dense dans $X_{0}^{\perp}$ : si $P_{0}$ est le projecteur oblique de $L^{2}$ sur $X_{0}$

$$
P_{0} f=\sum_{k \in \mathbb{Z}}\left\langle f, \omega_{0}(x-k)\right\rangle \varphi_{0}(x-k),
$$

alors $X_{0}^{\perp}=\left(\mathrm{Id}-P_{0}^{*}\right) L^{2}$ et $X_{0}^{\perp} \cap L_{\text {comp }}^{2}=\left(\operatorname{Id}-P_{0}^{*}\right) L_{\text {comp }}^{2}$. Maintenant, si $f \in V_{0}[\mu]$, alors $f \in\left(X_{0}^{\perp} \cap L_{\text {comp }}^{2}\right)^{\perp}$, et donc $f \in X_{0}$. L'inclusion $X_{0} \subset V_{0}[\mu]$ est évidente (il suffit de vérifier que $V_{0}[\mu]$ est fermé, ou encore que $V_{0}[\mu]=V_{0}\left[\varphi_{0}\right]$ : mais si $T \in L^{2}(\mathbb{R} / 2 \pi \mathbb{Z})$ et $P \in \mathbb{C}[X]$, il existe $S \in \mathcal{D}^{\prime}(\mathbb{R} / 2 \pi \mathbb{Z})$ tel que $T=P\left(e^{-i \xi}\right) S(\xi)$, et cela entraîne $\left.V_{0}\left[\varphi_{0}\right] \subset V_{0}[\mu]\right)$.

Nous comprenons maintenant pourquoi nous pouvons parler d'analyse multi-résolution:

\section{Lemme 7.}

i) $V_{j}[\mu]$ est fermé dans $L^{2}$ et a une base de Riesz $\left\{2^{j / 2} \varphi_{j}\left(2^{j} x-\right.\right.$ $k)\}_{k \in \mathbb{Z}}$ telle que $\varphi_{j} \in L_{\text {comp }}^{2}$ et telle qu'il existe $\omega_{j} \in C_{c}^{\infty}$ avec

$$
\left\langle 2^{j / 2} \varphi_{j}\left(2^{j} x-k\right), 2^{j / 2} \omega_{j}\left(2^{j} x-k\right)\right\rangle=\delta_{k, \ell} .
$$

ii) $V_{j}[\mu] \subset V_{j+1}[\mu]$.

iii) $\bigcup_{j \geq 0} V_{j}[\mu]$ est dense dans $L^{2}$. 
Preuve. i) est évident par renormalisation du Lemme 6. ii) est une évidence par définition de $V_{j}[\mu]$. Pour iii), on note $E=\overline{\bigcup_{j \geq 0} V_{j}[\mu]}$. Alors $E$ est un sous-espace fermé de $L^{2}$, invariant par toute translation dyadique $k / 2^{j}, k \in \mathbb{Z}, j \in \mathbb{N}$, donc par toute translation réelle. Cela implique qu'il existe un ensemble mesurable $F \subset \mathbb{R}$ tel que: $f \in E$ si et seulement si $\left\{f \in L^{2}\right.$ et $\left.\operatorname{supp} \hat{f} \subset F\right\}$. Comme $\mu \in E$ et que $\hat{\mu}$ est une fonction analytique, on a $F=\mathbb{R}$ et $E=L^{2}$.

Ainsi, la fonction $\mu$ permet d'approximer dans $L^{2}$, par ses translatées dyadiques, toute fonction de carré intégrable. Un problème intéressant est alors de savoir à quelle vitesse cette approximation a lieu.

Definition 6. Une fonction $\mu \in L_{\mathbb{R}}^{2} \cap L_{\text {comp }}^{2}(\mathbb{R}), \mu \neq 0$, a une puissance d'approximation d'ordre $N$ si: il existe $C \geq 0, j_{N} \in \mathbb{N}$, pour tout $f \in H^{N}, j \geq j_{N}$,

$$
\left\|f-\Pi_{j} f\right\|_{2} \leq C 2^{-j N}\left\|f^{(N)}\right\|_{2},
$$

où $\Pi_{j}$ est le projecteur orthogonal de $L^{2}$ sur $V_{j}[\mu]$.

Par exemple, si $\mu$ est une fonction d'échelle associée à un filtre $m_{0}$ qui a un zéro d'ordre $N$ en $\pi$, alors $\mu$ a une puissance d'approximation d'ordre $N$. Les fonctions d'échelle non-stationnaires ont été introduite pour permettre des puissances d'approximation d'ordre infini [11]. On parle alors d'approximation spectrale.

Remarquons également que si $\mu$ a une puissance d'approximation d'ordre $N, N \geq 1$, alors on a

$$
\lim _{j \rightarrow+\infty} 2^{j(N-1)}\left\|f-\Pi_{j} f\right\|_{2}=0, \quad \text { pour tout } f \in H^{N-1} .
$$

En effet, si $f=g_{A}+h_{A}$, avec

$$
\hat{g}_{A}(\xi)=\chi_{[-1,1]}\left(\frac{\xi}{A}\right) \hat{f}
$$

on a, en écrivant $g_{A} \in H^{N}$ et $h_{A} \in L^{2}$ avec

$$
\left\|g_{A}^{(N)}\right\|_{2} \leq A\left\|f^{(N-1)}\right\|_{2}
$$

et

$$
\left\|h_{A}\right\|_{2} \leq A^{-N+1}\left\|f^{(N-1)}\right\|_{2}
$$




$$
\begin{aligned}
2^{j(N-1)}\left\|f-\Pi_{j} f\right\|_{2} \leq & C\left(2^{-j} A\right)\left\|f^{(N-1)}\right\|_{2} \\
& +2\left(2^{j(N-1)} A^{-N+1}\right)\left\|f^{(N-1)}\right\|_{2},
\end{aligned}
$$

d'où, si $A=2^{j}$,

$$
2^{j(N-1)}\left\|f-\Pi_{j} f\right\|_{2} \leq(C+2)\left\|f^{(N-1)}\right\|_{2} .
$$

On réinjecte cette inégalité dans $f=g_{A}+h_{A}$, avec $g_{A} \in H^{N}$ et $h_{A} \in$ $H^{N-1}, A=(\sqrt{2})^{j}$

$$
\begin{aligned}
2^{j(N-1)}\left\|f-\Pi_{j} f\right\|_{2} \leq & C(\sqrt{2})^{-j}\left\|f^{(N-1)}\right\|_{2} \\
& +(C+2)\left(\int_{|\xi|>(\sqrt{2})^{j}}\left|f^{(N-1)}(\xi)\right|^{2} d \xi\right)^{1 / 2}
\end{aligned}
$$

et on a bien $2^{j(N-1)}\left\|f-\Pi_{j} f\right\|_{2} \rightarrow 0$ quand $j \rightarrow+\infty$.

Dans une analyse multi-résolution non stationnaire $\left(V_{j}[\mu]\right)$, nous avons obtenu pour chaque niveau $V_{j}[\mu]$ une fonction spéciale $\varphi_{j}$, et l'inclusion $V_{j}[\mu] \subset V_{j+1}[\mu]$ donne une équation à deux échelles

$$
\varphi_{j}\left(\frac{x}{2}\right)=\sum_{k \in \mathbb{Z}}\left\langle\varphi_{j}\left(\frac{x}{2}\right), \omega_{j+1}(x-k)\right\rangle \varphi_{j+1}(x-k),
$$

qui se réécrit en

$$
\hat{\varphi}_{j}(2 \xi)=\frac{1}{2} P_{\varphi_{j}(x / 2), \omega_{j+1}}(\xi) \hat{\varphi}_{j+1}(\xi)
$$

On écrit

$$
m_{j+1}(\xi)=\frac{1}{2} P_{\varphi_{j}(x / 2), \omega_{j+1}}(\xi)
$$

et on a alors

$$
\hat{\varphi}_{0}(\xi)=\prod_{j=1}^{M} m_{j}\left(\frac{\xi}{2^{j}}\right) \hat{\varphi}_{M}\left(\frac{\xi}{2^{M}}\right)
$$

formule qui est très proche de la formule qui relie la fonction d'échelle au filtre d'échelle. Mais ici, a priori, nous ne pouvons pas faire tendre $M$ vers $+\infty$. Même si nous pouvions normaliser $\varphi_{M} \operatorname{par} \hat{\varphi}_{M}(0)=1$ (ce 
qui n'est pas toujours possible!), il n'y aurait aucune raison de conclure que

$$
\lim _{M \rightarrow+\infty} \hat{\varphi}_{M}\left(\frac{\xi}{2^{M}}\right)=1 .
$$

Lorsque cela est le cas, nous sommes dans le cas d'analyses multirésolutions non-stationnaires "normales" (au sens où seuls les filtres $m_{j}$ entrent en compte pour la détermination de $\left.\varphi_{0}\right)$.

Definition 7. Une analyse multi-résolution non-stationnaire $\left\{V_{j}[\mu]\right\}_{j \geq 0}$ est dite normale si les fonctions d'échelle non-stationnaires $\varphi_{j}$ (i.e. les fonctions $\varphi_{j}$ telles que $\varphi_{j}\left(2^{j} x\right) \in V_{j}[\mu]$ et le support de $\varphi_{j}\left(2^{j} x\right)$ est minimum), peuvent être choisies de la forme

$$
\hat{\varphi}_{j}(\xi)=\prod_{\ell=1}^{\infty} m_{j+\ell}\left(\frac{\xi}{2^{\ell}}\right)
$$

où $m_{\ell}$ est un polynôme trigonométrique

$$
m_{\ell}=-\sum_{-\operatorname{deg} m_{\ell}}^{+\operatorname{deg} m_{\ell}} a_{k, \ell} e^{-i k \xi}
$$

à coefficients réels tel que $m_{\ell}(0)=1, \operatorname{deg} m_{\ell} \leq C \ell^{A}$ (où $A \in \mathbb{N}$ ne dépend pas de $\ell$ ) et

$$
\sup _{\ell}\left\|m_{\ell}\right\|_{\infty}=B<+\infty
$$

Comme nous l'avons expliqué dans l'introduction, les produits partiels

$$
\widehat{\theta_{j, N}}(\xi)=\prod_{\ell=1}^{N} m_{j+\ell}\left(\frac{\xi}{2^{\ell}}\right)
$$

définissant $\hat{\varphi}_{j}$ admettent un majorant à croissance polynômiale, de sorte que $\widehat{\theta_{j, N}}$, qui converge vers $\hat{\varphi}_{j}$ dans $C^{\infty}(\mathbb{R})$ (i.e., pour tout $p \in \mathbb{N}$, $(d / d \xi)^{p} \widehat{\theta_{j, N}}$ converge vers $(d / d \xi)^{p} \hat{\varphi}_{j}$ uniformément sur tout compact) converge vers $\hat{\varphi}_{j}$ également au sens des distributions tempérées. De plus $\operatorname{supp} \theta_{j, N} \subset\left[-M_{j}, M_{j}\right]$ avec

$$
M_{j}=\sum_{\ell=1}^{\infty} \frac{\operatorname{deg}\left(m_{j+\ell}\right)}{2^{\ell}} \leq C \sum_{\ell=1}^{\infty} \frac{(j+\ell)^{A}}{2^{\ell}} .
$$


La convergence de $\theta_{j, N}$ vers $\varphi_{j}$ a alors lieu avec des supports contenus dans un compact fixe (i.e., fixe par rapport à $N$, pas par rapport à $j$ ).

Nous verrons dans la suite trois exemples de telles analyses multirésolution non stationnaires: fonction de Rvachev (Section 5), base de Berkolaïko et Novikov (Section 6) et "ondelettes de Kharkov" (Section $8)$.

\section{La fonction de Rvachev.}

La fonction $u p(x)$ a été introduite en 1971 par V. A. et V. L. Rvachev comme une fonction explicite $C^{\infty}$, à support dans $[-1,1]$ et dont les translatées par $1 / 2^{j},\left\{u p\left(x-k / 2^{j}\right)\right\}_{k \in \mathbb{Z}}$, permettent de reconstruire tous les polynômes jusqu'au degré $j$

$$
\mathbb{C}_{j}[X] \subset\left\{\sum_{k \in \mathbb{Z}} a_{k} u p\left(x-\frac{k}{2^{j}}\right):\left\{a_{k}\right\} \in \mathbb{C}^{\mathbb{Z}}\right\}
$$

Nous translaterons cette fonction de 1 (c'est-à-dire que nous prendrons up à support dans $[0,2]$ ) pour pouvoir appliquer le formalisme des analyses multirésolutions non stationnaires.

La fonction $u p(x)$ peut être définie comme un exemple classique de fonction $C^{\infty}$ à support compact par la formule

$$
\widehat{u p}(\xi)=\prod_{j=0}^{\infty} \hat{\chi}\left(\frac{\xi}{2^{j}}\right)
$$

où $\chi=\chi_{[0,1]}$ et donc

$$
\hat{\chi}(\xi)=\frac{1-e^{-i \xi}}{i \xi} .
$$

De tels produits interviennent dans la théorie des classes de fonctions non quasi-analytiques ( $c f$. le livre de Rudin [25, Chapter 19, p. 415], par exemple).

Il y a de nombreuses façons de justifier la convergence de (20). Par exemple,

$$
\prod_{j=0}^{N} \hat{\chi}\left(\frac{\xi}{2^{j}}\right)=\hat{\mu}_{N}
$$


est la transformée de Fourier de la fonction $\mu_{N}=\chi * 2 \chi(2 x) * \cdots *$ $2^{N} \chi\left(2^{N} x\right)$, qui est une fonction positive de masse totale 1 et à support contenu dans $[0,2]$. De plus, $\left\|\mu_{N}\right\|_{\infty} \leq 1$ (puisque

$$
\left.\left\|\mu_{N}\right\|_{\infty}=\left\|\chi * 2 \mu_{N-1}(2 x)\right\|_{\infty} \leq\|\chi\|_{\infty}\left\|\mu_{N-1}\right\|_{1}\right)
$$

$\left\|(d / d x) \mu_{N}\right\|_{\infty} \leq 2$ (puisque $d \mu_{N} / d x=2 \mu_{N-1}(2 x)-2 \mu_{N-1}(2 x-$ $2)$ et que $\mu_{N-1}(x)$ et $\mu_{N-1}(x-2)$ sont à support dans $[0,2]$ et $[2,4]$ respectivement) et enfin

$$
\begin{aligned}
\| \mu_{N+1}- & \mu_{N} \|_{\infty} \\
& =\sup _{x}\left|\int\left(\mu_{N}(x)-\mu_{N}(x-y)\right) 2^{N+1} \chi\left(2^{N+1} y\right) d y\right| \leq 2^{-N} .
\end{aligned}
$$

$\mu_{N}$ converge donc uniformément vers une fonction up qui vérifie

$$
\text { supp up } \subset[0,2], \quad \int u p d x=1, \quad \text { et } u p=\chi * 2 u p(2 x) .
$$

En particulier, up est en quelque sorte sa propre régularisée et est dans $C^{\infty}$.

La fonction up jouit de nombreuses propriétés remarquables, décrites dans [26] et [27]. Par exemple, il est clair que

$$
\frac{d}{d x} u p(x)=2 u p(2 x)-2 u p(2 x-2)
$$

et que $u p(x)>0$ sur $] 0,2[, u p(1)=1$. (En effet, de (21) on tire que $0=\inf \operatorname{supp} u p$, et donc $u p(x)>0$ sur $] 0,1]$ tandis que $u p(1)=$ $\left.2 \int_{0}^{1} u p(2 t) d t=\widehat{u p}(0)=1\right)$. On en conclut alors pour $k \geq 1$

$$
0<x<2 \quad \text { et } \quad \frac{d^{k}}{d x^{k}} u p(x)=0,
$$

si et seulement si

$$
x \in\left\{\frac{1}{2^{k-1}}, \frac{2}{2^{k-1}}, \ldots, \frac{2^{k}-1}{2^{k-1}}\right\} .
$$

En fait c'est l'une des caractérisations de up

Proposition 4 (Caractérisation de up). Chacun des problèmes $\mathrm{P} 1$ ) à P5) a pour seule et unique solution la fonction up: 
P1) $f \in \mathcal{E}^{\prime},\langle f, 1\rangle=1$ et $f=\chi_{[0,1]} * 2 f(2 x)$

P2) $f \in \mathcal{E}^{\prime},\langle f, 1\rangle=1$ et $d f / d x=2 f(2 x)-2 f(x-2)$

P3) $f \in C^{\infty}, \operatorname{supp} f \subset[0,2], f(1)=1$ et pour tout $k \geq 1$ on a:

$$
0<x<2 \quad \text { et } \quad f^{(k)}(x)=0
$$

si et seulement si

$$
x \in\left\{\frac{1}{2^{k-1}}, \frac{2}{2^{k-1}}, \ldots, \frac{2^{k}-1}{2^{k-1}}\right\} .
$$

P4) $f \in C^{\infty}$, supp $f \subset[0,2], f(1)=1$,

$$
\lim _{n \rightarrow+\infty} 2^{-(n+1)(n+2) / 2}\left\|f^{(n)}\right\|_{\infty}=0
$$

et, pour tout $p \in \mathbb{N}$, il existe $\left\{a_{k}\right\} \in \mathbb{C}^{\mathbb{Z}}$, tel que

$$
x^{p}=\sum_{k \in \mathbb{Z}} a_{k} f\left(x-\frac{k}{2^{p}}\right) .
$$

P5) $f \in C^{\infty}, \operatorname{supp} f \subset[0,2], f(1)=1$,

$$
\lim _{n \rightarrow+\infty} 2^{-(n+1)(n+2) / 2}\left\|f^{(n)}\right\|_{\infty}=0
$$

et, pour tous $k \geq 1, \ell \in\left\{1,2, \ldots, 2^{k}-1\right\}$,

$$
f^{(k)}\left(\frac{\ell}{2^{k-1}}\right)=0
$$

Quoique tous ces résultats aient été prouvés par V. A. et V. L. Rvachev [26], [27], nous en donnerons la preuve à titre d'introduction à la fonction up. Avant d'entamer cette preuve, nous donnons le lemme fondamental sur lequel repose une grande partie de la théorie de la fonction $u p$.

Lemme 8. a) (Lemme d'encadrement) Soit $n \geq 0$ et soit $A_{n}$ l'ensemble des fonctions $f$ qui vérifient:

i) $f \in C^{n}$ et supp $f \subset[0,2]$. 
ii) $f^{(n)}$ est strictement monotone sur chaque intervalle $\left[k / 2^{n},(k+\right.$ 1) $\left./ 2^{n}\right]\left(0 \leq k<2^{n+1}\right)$.

iii) Pour $1 \leq p \leq n$ et $1 \leq k \leq 2^{p}-1$,

$$
f^{(p)}\left(\frac{k}{2^{p-1}}\right)=0 \text {. }
$$

iv) $f(1)=1$.

Alors si $f \in A_{n}$, on a en tout point $x$

$$
\ell_{n}(x) \leq f(x) \leq h_{n}(x),
$$

où $\ell_{0}=0, h_{0}=\chi_{[0,2]}$ et $\ell_{n+1}$ et $h_{n+1}$ sont définis par $\ell_{n+1}=\int_{0}^{x} L_{n}(t) d t$ et $h_{n+1}=\int_{0}^{x} H_{n}(t) d t$, où:

- pour $0 \leq x \leq 1 / 2, L_{n}(t)=2 \ell_{n}(2 t), H_{n}(t)=2 h_{n}(2 t)$,

- pour $1 / 2 \leq x \leq 1, L_{n}(t)=2 h_{n}(2 t), H_{n}(t)=2 \ell_{n}(2 t)$,

- pour $1 \leq x \leq 3 / 2, L_{n}(t)=-2 h_{n}(2 t-2), H_{n}(t)=-2 \ell_{n}(2 t-2)$,

- pour $3 / 2 \leq x \leq 2, L_{n}(t)=-2 \ell_{n}(2 t-2), H_{n}(t)=-2 h_{n}(2 t-2)$.

En particulier, on a: si $f, g \in A_{n}$, alors,

$$
\|f-g\|_{\infty} \leq\left\|h_{n}-\ell_{n}\right\|_{\infty} \leq \frac{2}{2^{n}} .
$$

b) (Lemme d'extremalité) Soit $\sigma_{n}$ le spline de degrén, défini récursivement par

$$
\sigma_{0}=\frac{1}{2} \chi_{[0,2]}
$$

et

$$
\sigma_{n+1}=\int_{0}^{x}\left(2 \sigma_{n}(2 t)-2 \sigma_{n}(2 t-2)\right) d t .
$$

Alors $\sigma_{n+1} \in A_{n}$ et on a, pour tout $f \in A_{n}$,

$$
\left\|f^{(n+1)}\right\|_{\infty} \geq\left\|\sigma_{n+1}^{(n+1)}\right\|_{\infty}=\frac{1}{2} 2^{(n+1)(n+2) / 2}
$$

et l'égalité n'a lieu que pour $f=\sigma_{n+1}$.

c) (Lemme de Majoration) Soit $f \in C^{n}(\mathbb{R})$ telle que $f^{(n)}$ soit lipschitzienne et telle que $f(k)=0$ pour tout $k \in \mathbb{Z}$ et $f^{(p)}\left(k / 2^{p-1}\right)=0$ pour tout $k \in \mathbb{Z}$ et tout $p \in\{1, \ldots, n\}$. Alors on a

$$
\|f\|_{\infty} \leq 2^{1-n-((n+1)(n+2)) / 2}\left\|f^{(n+1)}\right\|_{\infty} .
$$


Preuve Du Lemme 8. Il est immédiat que si $f \in A_{n}$, alors on a

$$
f^{\prime}(x)=f^{\prime}\left(\frac{1}{2}\right) f_{1}(2 x)+f^{\prime}\left(\frac{3}{2}\right) f_{2}(2 x-2),
$$

où $f_{1}$ et $f_{2}$ sont dans $A_{n-1}$. L'idée est alors de démontrer (22) par récurrence. On commence par vérifier que si $f \in A_{n}$, alors pour $1 \leq$ $p \leq n,\left\{f^{(p)}(x)=0\right.$ et $\left.0<x<2\right\}$ si et seulement si

$$
x \in\left\{\frac{1}{2^{p-1}}, \frac{2}{2^{p-1}}, \ldots, \frac{2^{p}-1}{2^{p-1}}\right\},
$$

par hypothèse, puisque

$$
f^{(n)}\left(\frac{k}{2^{n-1}}\right)=f^{(n)}\left(\frac{k+1}{2^{n-1}}\right)=0
$$

et que $f^{(n)}$ est strictement monotone sur $\left[2 k / 2^{n},(2 k+1) / 2^{n}\right]$ et sur $\left[2 k+1 / 2^{n},(2 k+2) / 2^{n}\right]$, on voit que $f^{(n)}$ ne s'annule pas sur $] k / 2^{n-1},(k+$ 1) $/ 2^{n-1}$ [ et donc que $f^{(n-1)}$ y est strictement monotone; ce qui prouve $A_{n} \subset A_{n-1}$ et par récurrence que les seuls zéros de $f^{(p)}$ sont les points $k / 2^{p-1}$.

Pour démontrer (22), Rvachev introduit l'hypothèse de récurrence $\left(H_{n}\right)$ suivante: "Si $f \in A_{n}, \alpha, \beta>0$ et $x_{0} \in[0,2]$ vérifient $\alpha \ell_{n}\left(x_{0}\right) \leq$ $f\left(x_{0}\right) \leq \beta h_{n}\left(x_{0}\right)$ alors si $x_{0} \leq 1$, on a $\alpha \ell_{n}(x) \leq f(x) \leq \beta h_{n}(x)$ pour tout $x \in\left[0, x_{0}\right]$, et si $x_{0} \geq 1$, on a $\alpha \ell_{n}(x) \leq f(x) \leq \beta h_{n}(x)$ pour tout $x \in\left[x_{0}, 2\right] "$.

$H_{0}$ est évident: puisque $\ell_{0}=0$, on a $f(x) \geq \alpha \ell_{0}(x)$ sur $[0,2]$ : en effet $f$ est croissante de 0 à 1 sur $[0,1]$ et décroissante de 1 à 0 sur $[1,2]$; de même si $f\left(x_{0}\right) \leq \beta h_{0}\left(x_{0}\right)=\beta$, on a nécessairement $f(x) \leq \beta$ sur $\left[0, x_{0}\right]$ si $x_{0} \leq 1$, sur $\left[x_{0}, 2\right]$ si $x_{0} \geq 1$.

Montrons que $\left(H_{n}\right)$ implique $\left(H_{n+1}\right)$. Comme 1 est centre de symétrie du problème (pour tout $n$ on a $f(x) \in A_{n}$ si et seulement si $f(2-x) \in A_{n}, \ell_{n}(x)=\ell_{n}(2-x)$ et $\left.h_{n}(x)=h_{n}(2-x)\right)$, nous pouvons supposer $x_{0} \leq 1$. On suppose donc $f \in A_{n+1}, x_{0} \leq 1$ et $\alpha \ell_{n+1}\left(x_{0}\right) \leq f\left(x_{0}\right) \leq \beta h_{n+1}\left(x_{0}\right)$. Sur $[0,1], f^{\prime}(x)=f^{\prime}(1 / 2) g(2 x)$ avec $f^{\prime}(1 / 2)>0$ et $g \in A_{n}$. On considère $\varphi(x)=f(x)-\alpha \ell_{n+1}(x)$. Cette fonction vérifie $\varphi\left(x_{0}\right) \geq 0$ et $\varphi(0)=0$. On note $x_{1}$ le point de $\left[0, x_{0}\right]$ où $\varphi$ atteint son minimum. Si $x_{1}=0$ ou $x_{1}=x_{0}$, alors $\varphi \geq 0$ sur $\left[0, x_{0}\right]$, ce qu'il faut démontrer. Si $0<x_{1}<1 / 2$, alors $0=\varphi^{\prime}\left(x_{1}\right)=f^{\prime}(1 / 2) g\left(2 x_{1}\right)-2 \alpha \ell_{n}\left(2 x_{1}\right)$. L'hypothèse $\left(H_{n}\right)$ permet de conclure que $\varphi^{\prime}(x) \geq 0$ sur $\left[0, x_{1}\right]$ et donc $\varphi\left(x_{1}\right) \geq \varphi(0)=0$. De même, 
si $1 / 2<x_{1}<1$, alors $0=\varphi^{\prime}\left(x_{1}\right)=f^{\prime}(1 / 2) g\left(2 x_{1}\right)-2 \alpha h_{n}\left(2 x_{1}\right)$, de sorte que $\varphi^{\prime}(x) \leq 0$ sur $\left[x_{1}, 1\right]$ et donc $\varphi\left(x_{1}\right) \geq \varphi\left(x_{0}\right) \geq 0$. Enfin si $x_{1}=1 / 2$, le même argument s'applique si $n+1 \geq 2$; si $n=0, \ell_{1}$ n'est pas dérivable en $1 / 2$; cependant $\ell_{1}(1 / 2)=0$ et $f(1 / 2)>0$, de sorte que $\varphi(1 / 2)>0$. $\left(H_{n+1}\right)$ est donc démontrée, le cas $f\left(x_{0}\right) \leq \beta h_{n+1}\left(x_{0}\right)$ se traitant de façon analogue.

(22) est alors immédiat. Pour $n=0$, nous avons déjà vu que $0 \leq f \leq \chi_{[0,2]}$. Pour $n \geq 1$, on a $f(1)=\ell_{n}(1)=h_{n}(1)$ et $\left(H_{n}\right)$ entraîne (22). Pour vérifier que $\ell_{n}(1)=h_{n}(1)=1$, il suffit de remarquer que pour $t \in[0,1]$ on a $\ell_{n}(t)+h_{n}(1-t)=1$. C'est vrai pour $n=0$. Par ailleurs, on a

$$
\begin{aligned}
\ell_{n+1}(t)+h_{n+1}(1-t)= & \int_{0}^{t} 2 \ell_{n}(2 u) d u+\int_{0}^{1 / 2} 2 h_{n}(2 u) d u \\
& +\int_{1 / 2}^{1-t} 2 \ell_{n}(2 u) d u \\
= & \int_{0}^{1} \ell_{n}(u) d u+\int_{0}^{1 / 2} h_{u}(u) d u \\
= & \int_{0}^{1}\left(\ell_{n}(u)+h_{n}(1-u)\right) d u=1, \quad \text { si } t \leq \frac{1}{2} .
\end{aligned}
$$

En utilisant le fait que $\ell_{n}(2-u)=\ell_{n}(u)$ et donc

$$
\int_{1 / 2}^{1-t} 2 \ell_{n}(2 u) d u=\int_{t}^{1 / 2} 2 \ell_{n}(2 v) d v
$$

et de même $\ell_{n+1}(t)+h_{n+1}(1-t)=1$ si $t \geq 1 / 2$.

Pour démontrer a), il ne reste plus qu'à estimer $\left\|\ell_{n}-h_{n}\right\|_{\infty}$. En fait, on va montrer que pour $0 \leq x \leq 1, \ell_{n}(x)=h_{n}\left(x-1 / 2^{n}\right)$. On commence par remarquer que c'est vrai pour $n=0$ ou 1. Par ailleurs, $\ell_{n}(x)=0$ pour $x \in\left[0,1 / 2^{n}\right]$ et donc $h_{n}=1$ pour $x \in\left[1-1 / 2^{n}, 1+1 / 2^{n}\right]$. On va montrer par récurrence que $\varphi_{n}(x)=\ell_{n}(x)-h_{n}\left(x-1 / 2^{n}\right)$ est nulle sur $[0,1)$. En effet, on a:

- si $0 \leq x \leq 1 / 2$,

$$
\varphi_{n+1}^{\prime}(x)=2 \ell_{n}(2 x)-2 h_{n}\left(2 x-\frac{1}{2^{n}}\right)=2 \varphi_{n}(2 x)=0,
$$


- si $1 / 2+1 / 2^{n+1} \leq x \leq 1$,

$$
\begin{aligned}
\varphi_{n+1}^{\prime}(x) & =\left\{\begin{array}{l}
2 h_{n}(2 x)-2 \ell_{n}\left(2 x-\frac{1}{2^{n}}\right), \\
2 h_{n}(2-2 x)-2 \ell_{n}\left(2-2 x+\frac{1}{2^{n}}\right),
\end{array}\right. \\
& =\left\{\begin{array}{l}
-2 \varphi_{n}\left(2-2 x+\frac{1}{2^{n}}\right), \\
0,
\end{array}\right.
\end{aligned}
$$

- si $1 / 2 \leq x \leq 1 / 2+1 / 2^{n+1}$,

$$
\varphi_{n+1}^{\prime}(x)=2 h_{n}(2 x)-2 h_{n}\left(2 x-\frac{1}{2^{n}}\right)=2-2=0,
$$

de sorte que $\varphi_{n+1}=\varphi_{n+1}(0)=0$.

On remarque alors que

$$
\left\|h_{n}-\ell_{n}\right\|_{\infty}=\left|h_{n}\left(\frac{1}{2}\right)-\ell_{n}\left(\frac{1}{2}\right)\right|=\int_{1 / 2-1 / 2^{n}}^{1 / 2} 2 h_{n-1}(2 t) d t \leq \frac{2}{2^{n}} .
$$

Le point a) est donc démontré.

Le point b) est quasiment immédiat. L'appartenance de $\sigma_{n+1}$ à $A_{n}$ est immédiate (il suffit d'écrire $\sigma_{n+1}=\chi_{[0,1]} * 2 \sigma_{n}(2 x)$ pour vérifier que pour tout $n \geq 0, \int \sigma_{n} d t=1$ et donc pour tout $n \geq 1, \sigma_{n}(1)=1$; les annulations de $\sigma_{n+1}^{(k)}$ sont alors immédiates par récurrence puisque $\sigma_{n+1}^{(k)}=2^{k} \sigma_{n}^{(k-1)}(2 t)$ sur $[0,1]$ et $=-2^{k} \sigma_{n}^{(k-1)}(2 t-2)$ sur $\left.[1,2]\right)$. De même il est immédiat que sur $] k / 2^{n},(k+1) / 2^{n}[$,

$$
\sigma_{n+1}^{(n+1)}=\varepsilon_{k, n} 2^{(n+1)(n+2) / 2}
$$

où $\varepsilon_{k, n}$ ne dépend que de $k$ et $n$ et appartient à $\{-1,1\}$. Le spline $\sigma_{n+1}$ est donc un spline parfait (i.e. un spline de degré $n+1$ tel que

$$
\left|\frac{d^{n+1}}{d x^{n+1}} \sigma_{n+1}\right|=c^{t e}
$$

presque partout sur son support). Il suffit alors de vérifier que si $\operatorname{supp} f \subset[0,2],\left\|f^{(n+1)}\right\|_{\infty} \leq\left\|\sigma_{n+1}^{(n+1)}\right\|_{\infty}$ et $f^{(p)}\left(k / 2^{p-1}\right)=0$ pour $0 \leq k \leq 2^{p}$ alors $|f(x)| \leq \sigma_{n+1}(x)$ en tout point et $f(1)=\sigma_{n+1}(1)=1$ si et seulement si $f=\sigma_{n+1}$. Cela est évident par récurrence. Si $n=0$, 
on a $\left|f^{\prime}\right| \leq 1$ presque pour tout et donc $\left|f(x)-f\left(x_{0}\right)\right| \leq\left|x-x_{0}\right|$; on prend $x_{0}=0$ pour $x \in[0,1]$ et $x_{0}=2$ pour $x \in[1,2]$ et on obtient $|f(x)| \leq(1-|1-x|)_{+}=\sigma_{1}(x)$; de plus on a

$$
|f(1)|=\left|\int_{0}^{1} f^{\prime}(t) d t\right| \leq \int_{0}^{1} 1 d t=1,
$$

avec égalité si et seulement si $f^{\prime}(t)=\omega$ presque pour tout sur $[0,1]$, où $|\omega|=1$ et $\omega$ ne dépend pas de $t$; on trouve donc $f(1)=1$ si et seulement si $f^{\prime}=1$ sur ]0,1[ et -1 sur ]1,2[, et donc si et seulement si $f=\sigma_{1}$.

Maintenant, si on suppose le résultat démontré pour $n$, il est facile de le démontrer pour $n+1$. En effet, puisque

$$
\left\|\sigma_{n+2}^{(n+2)}\right\|_{\infty}=2^{n+2}\left\|\sigma_{n+1}^{(n+1)}\right\|_{\infty}
$$

on écrit $f^{\prime} \chi_{[0,1]}=g(2 x)$ et $f^{\prime} \chi_{[1,2]}=h(2 x-2)$ et on peut appliquer la propriété au rang $n$ à $g$ et $h$ :

$$
\left\|g^{(n+1)}\right\|_{\infty}=2^{-(n+1)}\left\|f^{(n+2)}\right\|_{\infty} \leq 2\left\|\sigma_{n+1}^{(n+1)}\right\|_{\infty}
$$

et donc $\left|f^{\prime}(x)\right| \leq 2\left|\sigma_{n+1}(2 x)\right|$ sur $[0,1]$ et $\leq 2\left|\sigma_{n+1}(2 x-2)\right|$ sur [1,2]. En particulier,

$$
|f(x)| \leq \int_{0}^{x} 2 \sigma_{n+1}(2 t) d t=\sigma_{n+2}(x)
$$

si $0 \leq x \leq 1$, et

$$
|f(x)| \leq \int_{x}^{2} 2 \sigma_{n+1}(2 t-2) d t=\sigma_{n+2}(x)
$$

si $1 \leq x \leq 2$; de plus, l'égalité $f(1)=1=\sigma_{n+2}(1)$ n'est possible que si $f^{\prime}=2 \sigma_{n+1}(2 t)$ presque pour tout sur $[0,1]$ et $f^{\prime}=2 \sigma_{n+1}(2 t-$ 2) presque pour tout sur $[0,2]$, donc $f=\sigma_{n+2}$. Le point b) est donc démontré.

Le point c) est maintenant évident. Puisque $f^{(n)}$ est continue et que $f$ a un zéro d'ordre $(n+1)$ aux points entiers, on peut écrire

$$
f=\sum_{k=-\infty}^{+\infty} f_{k}(x-2 k)
$$


où $f_{k}$ est à support dans $[0,2], f_{k}^{(p)}\left(\ell / 2^{p-1}\right)=0$ pour $1 \leq p \leq n$, $1 \leq \ell \leq 2^{n}-1$ et $f_{k}(1)=0, f_{k}^{(n)}$ est lipschitzienne et $\left\|f_{k}^{(n+1)}\right\|_{\infty} \leq$ $\left\|f^{(n+1)}\right\|_{\infty}$. La fonction

$$
\tilde{f}_{k, \varepsilon}=\sigma_{n+1}+f_{k} \frac{\left\|\sigma_{n+1}^{(n+1)}\right\|_{\infty}}{\left\|f^{(n+1)}\right\|_{\infty}+\varepsilon}
$$

(où $\varepsilon>0$ ) appartient à $A_{n}$, de sorte que

$$
\left\|\tilde{f}_{k, \varepsilon}-\sigma_{n+1}\right\|_{\infty} \leq \frac{2}{2^{n}}
$$

Faisant tendre $\varepsilon$ vers 0 , on obtient

$$
\|f\|_{\infty}=\sup _{k}\left\|f_{k}\right\|_{\infty} \leq \frac{2}{2^{n}} 2^{-(n+1)(n+2) / 2}\left\|f^{(n+1)}\right\|_{\infty} .
$$

Le Lemme 8 est donc démontré.

Preuve de la proposition 4. On vérifie facilement que up est solution des problèmes $\mathrm{P} 1$ ) à $\mathrm{P} 5$ ). En effet, on a

$$
\frac{d}{d x} u p=2 u p(2 x)-2 u p(2 x-2)
$$

et

$$
\operatorname{supp} u p(2 x) \cap \operatorname{supp} u p(2 x-2)=\{1\} \text {. }
$$

On en conclut

$$
\left\|\frac{d^{N}}{d x^{N}} u p\right\|_{\infty}=2\left\|\frac{d^{N-1}}{d x^{N-1}}(u p(2 x))\right\|_{\infty}=2^{N}\left\|\frac{d^{N-1}}{d x^{N-1}} u p\right\|_{\infty},
$$

et donc

$$
\left\|\frac{d^{N}}{d x^{N}} u p\right\|_{\infty}=2^{-(N+1)} 2^{(N+1)(N+2) / 2} .
$$

Il reste seulement à vérifier que tout polynôme s'écrit à l'aide de translatées de up. On va montrer plus précisément, pour $p \in \mathbb{N}$, pour $\ell \in\{0, \ldots, p\}$,

$$
\sum_{k \in \mathbb{Z}}\left(\frac{k}{2^{p}}\right)^{\ell} u p\left(x-\frac{k}{2^{p}}\right)=2^{p} x^{\ell} \bmod \mathbb{C}_{\ell-1}[X] .
$$


Pour $p=0,(26)$ se réduit à $\sum_{k \in \mathbb{Z}} u p(x-k)=1$, ou encore à $\widehat{u p}(0)=1$ et $\widehat{u p}(2 k \pi)=0$ pour $k \in \mathbb{Z}^{*}$, ce qui est immédiat puisque

$$
\widehat{u p}(\xi)=\frac{1-e^{-i \xi}}{i \xi} \widehat{u p}\left(\frac{\xi}{2}\right)
$$

Si (26) est vrai à l'ordre $p$, cela l'est encore à l'ordre $p+1$ pour $0 \leq \ell \leq p$

$$
\begin{aligned}
\sum_{k \in \mathbb{Z}}\left(\frac{k}{2^{p+1}}\right)^{\ell} u p\left(x-\frac{k}{2^{p+1}}\right)= & \sum_{k \in \mathbb{Z}}\left(\frac{k}{2^{p}}\right)^{\ell} u p\left(x-\frac{k}{2^{p}}\right) \\
& +\sum_{k \in \mathbb{Z}}\left(\frac{k}{2^{p}}\right)^{\ell} u p\left(x-\frac{1}{2^{p+1}}-\frac{k}{2^{p}}\right) \\
& +\sum_{j=1}^{\ell}\left(\frac{1}{2^{p+1}}\right)^{j} C_{\ell}^{j} \\
& \cdot \sum_{k \in \mathbb{Z}}\left(\frac{k}{2^{p}}\right)^{\ell-j} u p\left(x-\frac{1}{2^{p+1}}-\frac{k}{2^{p}}\right) \\
= & 2^{p} x^{\ell}+2^{p}\left(x-\frac{1}{2^{p+1}}\right)^{\ell} \bmod \mathbb{C}_{\ell-1}[x] \\
= & 2^{p+1} x^{\ell} \bmod \mathbb{C}_{\ell-1}[X] .
\end{aligned}
$$

Pour $\ell=p+1$, on utilise le fait que $\widehat{u p}$ a un zéro d'ordre $p+2$ en $2^{p+2} k \pi, k \in \mathbb{Z}^{*}$, puisque

$$
\widehat{u p}(\xi)=\prod_{j=0}^{p+1} \frac{1-e^{-i \xi / 2^{j}}}{i \xi / 2^{j}} \widehat{u p}\left(\frac{\xi}{2^{p+2}}\right)
$$

la formule sommatoire de Poisson donne alors que

$$
\sum_{k \in \mathbb{Z}}\left(x-\frac{k}{2^{p+1}}\right)^{p+1} u p\left(x-\frac{k}{2^{p+1}}\right)=C^{t e}=\gamma_{p+1}
$$

où $\gamma_{p+1}$ est une constante ne dépendant pas de $x$. On obtient alors

$$
\begin{aligned}
0 & =\sum_{j=0}^{p+1} C_{p+1}^{j}(-1)^{j} x^{p+1-j} \sum_{k \in \mathbb{Z}}\left(\frac{k}{2^{p+1}}\right)^{j} u p\left(x-\frac{k}{2^{p+1}}\right) \bmod \mathbb{C}_{p}[X] \\
& =(-1)^{p+1} \sum_{k \in \mathbb{Z}}\left(\frac{k}{2^{p+1}}\right)^{p+1} u p\left(x-\frac{k}{2^{p+1}}\right)
\end{aligned}
$$




$$
\begin{aligned}
+ & \sum_{j=0}^{p} C_{p+1}^{j}(-1)^{j} x^{p+1-j} 2^{p+1} x^{j} \bmod \mathbb{C}_{p}[X] \\
=(-1)^{p+1} \sum_{k \in \mathbb{Z}} & \left(\frac{k}{2^{p+1}}\right)^{p+1} u p\left(x-\frac{k}{2^{p+1}}\right) \\
& -(-1)^{p+1} 2^{p+1} x^{p+1} \bmod \mathbb{C}_{p}[X] .
\end{aligned}
$$

(26) est donc prouvé.

up est donc bien solution des problèmes $\mathrm{P} 1$ ) à $\mathrm{P} 5$ ). Par ailleurs, les restrictions sur la taille des dérivées de $f$ dans $\mathrm{P} 4$ ) ou P5) sont bien nécessaires pour ne pas avoir d'autre solution que up: pour $\mathrm{P} 4)$, la fonction

$$
f=u p-\alpha \frac{d}{d x} u p,
$$

où $\alpha>0$ est arbitraire, vérifie supp $f \subset[0,2], \int_{0}^{2} f d x=1$ et

$$
\begin{aligned}
\sum_{k \in \mathbb{Z}}\left(\frac{k}{2^{p}}\right)^{p} f\left(x-\frac{k}{2^{p}}\right)= & \sum_{k \in \mathbb{Z}}\left(\frac{k}{2^{p}}\right)^{p} u p\left(x-\frac{k}{2^{p}}\right) \\
& -\alpha \frac{d}{d x}\left(\sum_{k \in \mathbb{Z}}\left(\frac{k}{2^{p}}\right)^{p} u p\left(x-\frac{k}{2^{p}}\right)\right) \\
= & 2^{p} x^{p} \bmod \mathbb{C}_{p-1}[X]
\end{aligned}
$$

tandis que

$$
\lim _{n \rightarrow+\infty} \frac{\left\|f^{(n)}\right\|_{\infty}}{2^{(n+1)(n+2) / 2}}=\alpha
$$

Pour P5), la fonction $f=u p(2 x-1)$ vérifie supp $f \subset[0,2], f(1)=1$ et

$$
f^{(k)}\left(\frac{\ell}{2^{k-1}}\right)=2^{k} u p^{(k)}\left(\frac{2 \ell}{2^{k-1}}-1\right)=0,
$$

tandis que

$$
\left\|f^{(n)}\right\|_{\infty}=2^{n} 2^{n(n+1) / 2}=\frac{1}{2} 2^{(n+1)(n+2) / 2} .
$$

Il reste maintenant à vérifier l'unicité des solutions de P1) à P5).

- Pour P1), c'est évident: si $f=\chi * 2 f(2 x)$, alors

$$
\hat{f}(\xi)=\hat{\chi}(\xi) \hat{f}\left(\frac{\xi}{2}\right)=\prod_{j=0}^{N} \hat{\chi}\left(\frac{\xi}{2^{j}}\right) \hat{f}\left(\frac{\xi}{2^{N+1}}\right)
$$


et donc

$$
\hat{f}(\xi)=\hat{f}(0) \prod_{j=0}^{\infty} \hat{\chi}\left(\frac{\xi}{2^{j}}\right)=\hat{f}(0) \widehat{u p}(\xi) .
$$

- Pour P2), on remarque que

$$
\frac{d}{d x}(\chi * 2 f(2 x))=2 f(2 x)-2 f(2 x-2),
$$

de sorte que pour $f \in \xi$ il est équivalent de dire que $f=\chi * 2 f(2 x)$ ou que

$$
\frac{d f}{d x}=2 f(2 x)-2 f(2 x-2) .
$$

- Pour P3), on utilise le Lemme 8: si $f$ vérifie les hypothèses de P3), alors $f \in A_{n}$ pour tout $n$. Comme $u p \in A_{n}$, le lemme d'encadrement donne que

$$
\|f-u p\|_{\infty} \leq\left\|h_{n}-\ell_{n}\right\|_{\infty} \leq \frac{2}{2^{n}} .
$$

Comme c'est vrai pour tout $n, f=u p$.

- Pour P4), on commence par remarquer la chose suivante: si $\mu \in C_{c}^{\infty}$ et si $\nu$ est la fonction de support minimum dans $V_{0}[\mu]$, avec $\omega$ une fonction dans $C_{c}^{\infty}$ vérifiant $\langle\omega(x-k), \nu\rangle=\delta_{k, 0}$ pour $k \in \mathbb{Z}$, alors le fait que $f \in L_{\text {loc }}^{2}$ s'écrive

$$
f=\sum_{k \in \mathbb{Z}} \alpha_{k} \mu(x-k), \quad \text { avec }\left\{\alpha_{k}\right\} \in \mathbb{C}^{\mathbb{Z}}
$$

entraîne que

$$
f=\sum_{k \in \mathbb{Z}}\langle f, \omega(x-k)\rangle \nu(x-k) .
$$

En particulier, si $1=\sum_{k \in \mathbb{Z}} \alpha_{k} \mu(x-k)$, on obtient que

$$
1=\langle 1, \omega\rangle \sum_{k \in \mathbb{Z}} \nu(x-k),
$$

et donc que $\sum_{k}(d \nu / d x)(x-k)=0$; cela entraîne que

$$
\frac{d \nu}{d x}=\alpha_{1}(x)-a_{1}(x-1)
$$


pour une fonction $\alpha_{1} \in C_{c}^{\infty}$, et donc que

$$
\hat{\mu}(\xi)=\frac{1-e^{-i \xi}}{i \xi} \hat{\mu}_{1}(\xi)
$$

pour une fonction $\mu_{1} \in C_{c}^{\infty}$. On a alors $V_{1}[d \mu / d x]=V_{1}\left[\mu_{1}\right]$. A nouveau, si $\nu_{1}$ est la fonction de support minimum de $V_{1}[d \mu / d x]$ et si $\omega_{1} \in C_{c}^{\infty}$ vérifie

$$
\left\langle\omega_{1}\left(x-\frac{k}{2}\right), \nu_{1}\right\rangle=\delta_{k, 0}
$$

on a pour

$$
f=\sum_{k \in \mathbb{Z}} \alpha_{k} \frac{d}{d x} \mu\left(x-\frac{k}{2}\right), \quad f=\sum\left\langle f, \omega_{1}\left(x-\frac{k}{2}\right)\right\rangle \nu_{1}\left(x-\frac{k}{2}\right) .
$$

Ainsi, si en plus de $1=\sum_{k \in \mathbb{Z}} \alpha_{k} \mu(x-k)$ on a

$$
x=\sum_{k \in \mathbb{Z}} \beta_{k} \mu\left(x-\frac{k}{2}\right),
$$

on écrit

$$
1=\sum_{k \in \mathbb{Z}} \beta_{k} \frac{d}{d x} \mu\left(x-\frac{k}{2}\right)
$$

et on obtient finalement

$$
\frac{d}{d x} \nu_{1}=\alpha_{2}(x)-\alpha_{2}\left(x-\frac{1}{2}\right)
$$

et enfin

$$
\hat{\mu}(\xi)=\frac{1-e^{-i \xi}}{i \xi} \frac{1-e^{-i \xi / 2}}{i \xi / 2} \hat{\mu}_{2}(\xi) .
$$

Cet argument s'itère à l'infini et donne donc que si $f$ vérifie les hypothèses de $\mathrm{P} 4$ ), on a pour tout $N \in \mathbb{N}$,

$$
\hat{f}(\xi)=\prod_{j=0}^{N} \frac{1-e^{-i \xi / 2^{j}}}{\left(i \xi / 2^{j}\right)} \hat{f}_{N+1}(\xi)
$$

avec $f_{N+1} \in C_{c}^{\infty}$. Le problème est alors de montrer que

$$
\lim _{N \rightarrow+\infty} \hat{f}_{N+1}(\xi)=1
$$


ponctuellement. En notant $f_{0}=f$, on a pour tout $N$,

$$
f_{N+1}=\frac{1}{2^{N}} \sum_{k \geq 0} \frac{d}{d x} f_{N}\left(x-\frac{k}{2^{N}}\right)=-\frac{1}{2^{N}} \sum_{k<0} \frac{d}{d x} f_{N}\left(x-\frac{k}{2^{N}}\right) .
$$

En particulier, on a: $\operatorname{supp} f_{N+1} \subset\left[\inf \operatorname{supp} f_{N}, \sup \operatorname{supp} f_{N}-1 / 2^{N}\right]=$ $\left[0,1 / 2^{N}\right]$, de sorte qu'en fait on a

$$
f_{N+1}=\chi_{\left[0,1 / 2^{N}\right]} \frac{1}{2^{N}} \frac{d}{d x} f_{N}=\chi_{\left[0,1 / 2^{N}\right]} 2^{-N(N+1) / 2}\left(\frac{d}{d x}\right)^{N+1} f,
$$

et donc

$$
\hat{f}_{N+1}(\xi)=2^{-N(N+1) / 2} \int_{0}^{1 / 2^{N}} f^{(N+1)}(x) e^{-i x \xi} d x
$$

Par ailleurs $\hat{f}_{N+1}(0)=1$ et donc

$$
\hat{f}_{N+1}(\xi)-1=2^{-N(N+1) / 2} \int_{0}^{1 / 2^{N}} f^{(N+1)}(x)\left(e^{-i x \xi}-1\right) d x,
$$

d'où

$$
\begin{aligned}
\left|\hat{f}_{N+1}(\xi)-1\right| & \leq 2^{-N(N+1) / 2} \int_{0}^{1 / 2^{N}}\left\|f^{(N+1)}\right\|_{\infty} \frac{|\xi|}{2^{N}} d x \\
& \leq 2^{-N(N+1) / 2} 2^{-N}\left\|f^{(N+1)}\right\|_{\infty} \frac{|\xi|}{2^{N}} \\
& =2^{-(N+2)(N+3) / 2}\left\|f^{(N+1)}\right\|_{\infty} 8|\xi|
\end{aligned}
$$

et on a bien $\lim _{N \rightarrow+\infty}\left|\hat{f}_{N+1}(\xi)-1\right|=0$. Enfin P5) est immédiat, en appliquant le Lemme 8 (lemme de majoration) à $f-u p$.

La Proposition 4 est donc démontrée.

Une propriété amusante de la fonction up est que ses valeurs aux points dyadiques sont rationnelles et aisément calculables. Ce qui en permet la tabulation et partant le calcul de toutes les quantités

$$
\int_{k / 2^{j}}^{(k+1) / 2^{j}} x^{\ell} u p(x) d x
$$


Lemme 9. Pour tous $j \in \mathbb{N}, k \in\left\{1, \ldots, 2^{j+1}-1\right\}, u p\left(k / 2^{j}\right) \in \mathbb{Q}$.

Preuve. On remarque d'abord que tous les moments

$$
m_{\ell}=\int_{0}^{2} x^{\ell} u p(x) d x
$$

sont à valeurs rationnelles. En effet, on a

$$
\begin{aligned}
\int_{0}^{2} x^{\ell} u p(x) d x= & -\int_{0}^{2} \frac{x^{\ell+1}}{\ell+1} \frac{d}{d x} u p(x) d x \\
= & \int_{0}^{2}(2 u p(2 x-2)-2 u p(2 x)) \frac{x^{\ell+1}}{\ell+1} d x \\
= & \int_{0}^{2} u p(t)\left(\left(\frac{t+2}{2}\right)^{\ell+1}-\left(\frac{t}{2}\right)^{\ell+1}\right) \frac{d t}{\ell+1} \\
= & \frac{1}{2^{\ell}} \int_{0}^{2} t^{\ell} u p(t) d t \\
& +\sum_{k=0}^{\ell-1} \frac{1}{\ell+1} C_{\ell+1}^{k} \frac{2^{\ell+1-k}}{2^{\ell+1}} \int_{0}^{2} t^{k} u p(t) d t .
\end{aligned}
$$

Partant de $\int_{0}^{2} u p(x) d x=1$, on voit que $\int_{0}^{2} t^{\ell} u p(t) d t \in \mathbb{Q}$ par induction sur $\ell$.

On calcule alors $u p\left(k / 2^{j}\right)$ par récurrence sur $j$ à l'aide des formules

$$
\sum_{k \in \mathbb{Z}}\left(\frac{x-k}{2^{j}}\right)^{j} u p\left(\frac{x-k}{2^{j}}\right)=\int\left(\frac{x}{2^{j}}\right)^{j} u p\left(\frac{x}{2^{j}}\right) d x=2^{j} m_{j} .
$$

Partant de $u p(1)=1$, on voit alors qu'on peut calculer les valeurs $u p\left((2 k-1) / 2^{j+1}\right)$ pour $1 \leq k \leq 2^{j+1}$ à l'aide des valeurs $u p\left(k / 2^{j}\right)$, $1 \leq k \leq 2^{j+1}-1$, en résolvant le système de $2^{j+1}$ équations à $2^{j+1}$ inconnues:

(S)

$$
\left\{\begin{array}{c}
\text { pour } 0 \leq \ell \leq j, \text { pour } 1 \leq r \leq 2^{j-\ell}, \\
\sum_{k=0}^{2^{\ell+1}-1}\left(\frac{2 r-1}{2^{j+1}}+\frac{k}{2^{\ell}}\right)^{\ell} u p\left(\frac{2 r-1}{2^{j+1}}+\frac{k}{2^{\ell}}\right)=2^{\ell} m_{\ell}, \\
\sum_{k=1}^{2^{j+1}}\left(\frac{2 k-1}{2^{j+1}}\right)^{j+1} u p\left(\frac{2 k-1}{2^{j+1}}\right) \\
=2^{j+1} m_{j+1}-\sum_{k=1}^{2^{j+1}-1}\left(\frac{k}{2^{j}}\right)^{j+1} u p\left(\frac{k}{2^{j}}\right) .
\end{array}\right.
$$


Il reste à vérifier que le système est de Cramer. Mais les $2^{j}$ équations correspondant à $\ell=0$ permettent d'exprimer $u p\left((2 r-1) / 2^{j+1}+1\right)$ en fonction de $u p\left((2 r-1) / 2^{j+1}\right)\left(1 \leq r \leq 2^{j}\right)$ et $(\mathrm{S})$ devient alors pour ces valeurs $u p\left((2 r-1) / 2^{j+1}\right)\left(1 \leq r \leq 2^{j}\right)$ :

$\left(\mathrm{S}^{\prime}\right)$

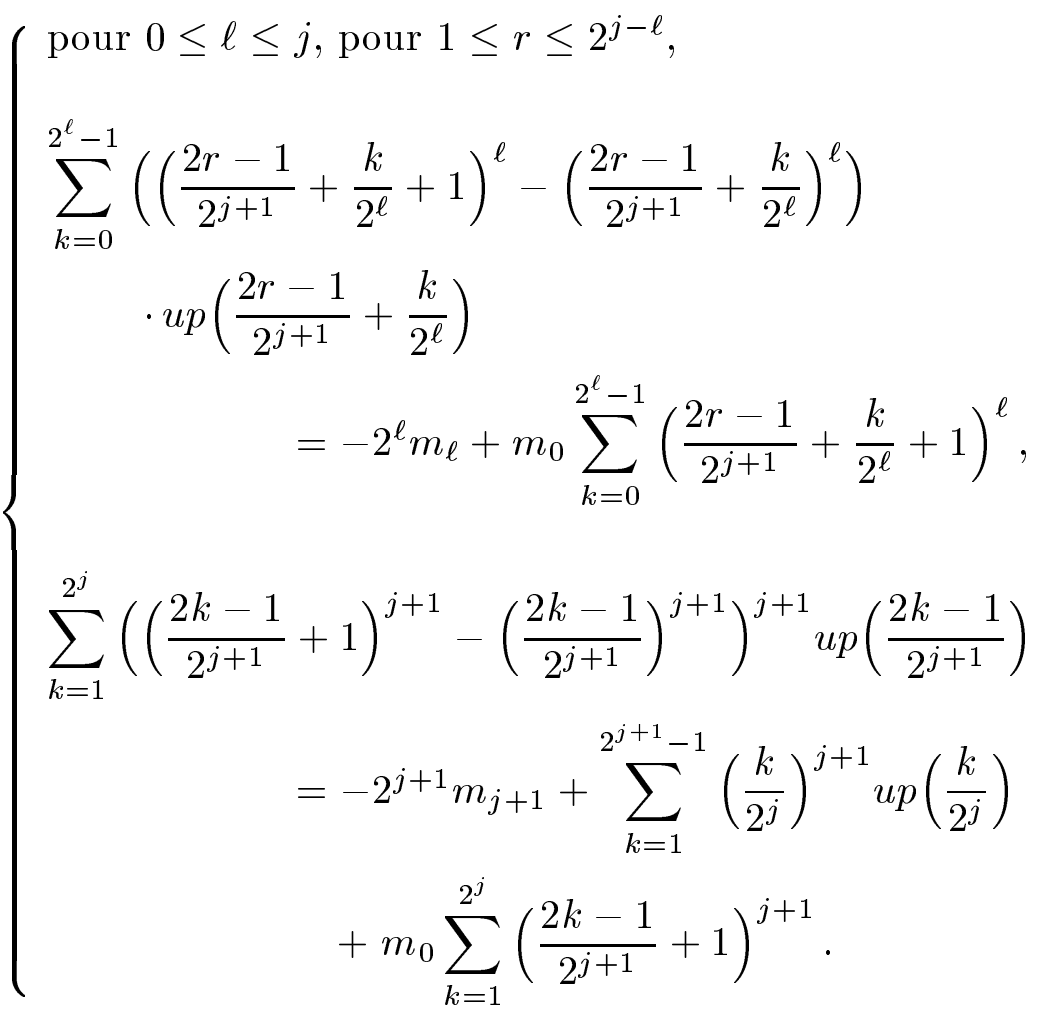

$\left(S^{\prime}\right)$ se réécrit alors,

$\left(\mathrm{S}^{\prime \prime}\right) \quad\left\{\begin{array}{l}\text { pour } 0 \leq \ell \leq j-1, \text { pour } 1 \leq r \leq 2^{j-1-\ell}, \\ \sum_{k=0}^{2^{\ell+1}-1}\left(\frac{2 r-1}{2^{j}}+\frac{k}{2^{\ell}}\right)^{\ell} u p\left(\frac{2 r-1}{2^{j+1}}+\frac{k}{2^{\ell+1}}\right)=c_{\ell, r}, \\ \sum_{\ell=1}^{2^{j}}\left(\frac{2 k-1}{2^{j}}\right)^{j} u p\left(\frac{2 k-1}{2^{j+1}}\right)=c_{j} .\end{array}\right.$

où les $c_{\ell, r}$ et $c_{j}$ sont des combinaisons linéaires des $u p\left(k / 2^{j}\right)$ à coefficients rationnels. On retrouve le système $(\mathrm{S})$ au rang $j$ (où les inconnues $u p\left((2 k-1) / 2^{j}\right)$ sont remplacées par les inconnues $\left.u p\left(1 / 2(2 k-1) / 2^{j}\right)\right)$ et par récurrence sur $j$, on obtient bien que $(S)$ est un système de Cramer. 
Nous pouvons maintenant présenter le résultat le plus frappant de Rvachev [26], [27]:

Proposition 5 (Série de Taylor généralisée). Pour $\rho \in] 1,2[$, on désigne par $K^{\rho}$ l'espace de Banach

$$
K^{\rho}=\left\{f \in C^{\infty}(\mathbb{R}): \sup _{N} \rho^{-N} 2^{-N(N+1) / 2}\left\|f^{(N)}\right\|_{\infty}<+\infty\right\} .
$$

Alors l'application

$$
f \longrightarrow\{f(k)\}_{k \in \mathbb{Z}} \bigcup\left\{\rho^{-N} 2^{-N(N+1) / 2} f^{(N)}\left(\frac{k}{2^{N-1}}\right)\right\}_{N \geq 1, k \in \mathbb{Z}}
$$

est un isomorphisme de $K^{\rho}$ sur $\ell^{\infty}\left(\mathbb{Z} \oplus \mathbb{N}^{*} \times \mathbb{Z}\right)$. Autrement dit toute fonction de $K^{\rho}$ se décompose en

$$
f=\sum_{k \in \mathbb{Z}} f(k) \varphi_{0, k}(x)+\sum_{N=1}^{\infty} \sum_{k \in \mathbb{Z}} f^{(N)}\left(\frac{k}{2^{N-1}}\right) \varphi_{N, k}(x),
$$

où $\varphi_{N, k}$ est l'unique solution de: $\varphi_{N, k} \in K^{\rho}, \varphi_{N, k}(\ell)=\delta_{N, 0} \delta_{k, \ell}$ et

$$
\left(\frac{d}{d x}\right)^{j} \varphi_{N, k}\left(\frac{\ell}{2^{j-1}}\right)=\delta_{N, j} \delta_{\ell, k},
$$

pour $j \geq 1$. La convergence de la série a lieu uniformément sur tout compact de $\mathbb{R}$ pour $f$ ainsi que pour toutes ses dérivées.

Preuve. Notons $S_{\rho}$ l'application

$$
f \longrightarrow\{f(k)\}_{k \in \mathbb{Z}} \bigcup\left\{\rho^{-N} 2^{-N(N+1) / 2} f^{(N)}\left(\frac{k}{2^{N-1}}\right)\right\}_{N \geq 1, k \in \mathbb{Z}} .
$$

Il est clair que $S_{\rho}$ est injective de $K^{\rho}$ dans $\ell^{\infty}\left(\mathbb{Z} \oplus \mathbb{N}^{*} \times \mathbb{Z}\right)$. En effet, si $S_{\rho} f=0$, le Lemme 8 (lemme de majoration) donne que pour tout $n$ on a

$$
\|f\|_{\infty} \leq 4\left(\frac{\rho}{2}\right)^{n+1} \rho^{-n-1} 2^{-(n+1)(n+2) / 2}\left\|f^{(n+1)}\right\|_{\infty} .
$$

Si $f \in K^{\rho}$, cela donne $\|f\|_{\infty} \leq C(\rho / 2)^{n+1}$ où $C$ ne dépend pas de $n$ et donc $f=0$.

Nous allons montrer maintenant l'existence de l'unique $\varphi_{N, k} \in K^{\ell}$ telle que

$$
S_{\rho}\left(\varphi_{N, k}\right)=\left\{\delta_{N, 0} \delta_{k, \ell}\right\}_{\ell \in \mathbb{Z}} \bigcup\left\{\rho^{-j} 2^{-j(j+1) / 2} \delta_{N, j} \delta_{k, \ell}\right\}_{j \geq 1, \ell \in \mathbb{Z}} .
$$


Nous montrerons de plus que $\varphi_{N, k} \in V_{N}[u p]$ et que pour $N \geq 1$,

$$
\operatorname{supp} \varphi_{N, k} \subset\left[-E\left(\frac{-k}{2^{N-1}}\right)-1, E\left(\frac{k}{2^{N-1}}\right)+1\right] .
$$

Nous allons construire $\varphi_{N, k}$ par récurrence sur $N$. Si $N=0$, on connaît déjà la solution: $\varphi_{0, k}=u p(x-1+k)$. Pour construire $\varphi_{N, k}$, une fois connues les $\varphi_{j, k}$ pour $j<N$, on part de l'égalité

$$
\frac{d^{N}}{d x^{N}} u p\left(\frac{1}{2^{N}}\right)=2^{N(N+1) / 2}
$$

et on procède en trois étapes:

- en notant $I_{N, k}$ l'intervalle

$$
I_{N, k}=\left[-E\left(\frac{-k}{2^{N-1}}\right)-1, E\left(\frac{k}{2^{N-1}}\right)+1\right],
$$

on définit $\omega_{N, k}$ par

$$
\omega_{N, k}=\sum_{\ell / 2^{N-1} \in I_{N, k} \text { et } \ell \geq k} c_{N, k, \ell} u p\left(x-\frac{\ell}{2^{N-1}}+\frac{1}{2^{N}}\right),
$$

où les $c_{N, k, \ell}$ sont choisis de sorte que: pour $\ell / 2^{N-1} \in I_{N, k}$ et $k \leq \ell$,

$$
\frac{d^{N}}{d x^{N}} \omega_{N, k}\left(\frac{\ell}{2^{N-1}}\right)=\delta_{k, \ell}
$$

C'est-à-dire qu'on prend $c_{N, k, k}=2^{-N(N+1) / 2}$ et pour $\ell>k$,

$$
c_{N, k, \ell}=-2^{-N(N+1) / 2} \sum_{m=k}^{\ell-1} c_{N, k, m} \frac{d^{N}}{d x^{N}} u p\left(\frac{\ell-m}{2^{N-1}}+\frac{1}{2^{N}}\right) .
$$

Les $c_{N, k, \ell}$ sont donc bien définis et on a

$$
\frac{d^{N}}{d x^{N}} \omega_{N, k}\left(\frac{\ell}{2^{N-1}}\right)=\delta_{k, \ell},
$$

pour tous les points $\ell / 2^{N-1} \in I_{N, k}$, tandis que pour tout $p>N$ et tout $\ell \in \mathbb{Z}$

$$
\frac{d^{p}}{d x^{p}} \omega_{N, k}\left(\frac{\ell}{2^{p-1}}\right)=0
$$


- On corrige alors $\omega_{N, k}$ en

$$
\begin{aligned}
\psi_{N, k}= & \omega_{N, k}-\sum_{\ell \in I_{N, k}} \omega_{N, k}(\ell) \varphi_{0, \ell}(x) \\
& -\sum_{p=1}^{N-1} \sum_{\ell / 2^{p-1} \in I_{N, k}} \frac{d^{p}}{d x^{p}} \omega_{N, k}\left(\frac{\ell}{2^{p-1}}\right) \varphi_{p, \ell}(x) .
\end{aligned}
$$

On a alors $\psi_{N, k}(\ell)=0$ pour $\ell \in I_{N, k}$ et

$$
\frac{d^{p}}{d x^{p}} \omega_{N, k}\left(\frac{\ell}{2^{p-1}}\right)=\delta_{N, p} \delta_{k, \ell}
$$

our $p \geq 1$ et $\ell / 2^{p-1} \in I_{N, k}$. En particulier $\psi_{N, k}$ a un zéro d'ordre infini aux deux bornes de $I_{N, k}$.

- Il suffit alors de poser: $\varphi_{N, k}=\psi_{N, k} \chi_{I_{N, k}}$.

Bien sûr, on peut remplacer dans ce raisonnement $I_{N, k}$ par n'importe quel intervalle $I \supset I_{N, k}$ à bornes dans $\mathbb{Z}$. On obtient alors $\varphi_{N, k}=$ $\omega_{I} \chi_{I}$ avec $\omega_{I} \in V_{N}[u p]$; cela entraîne que $\varphi_{N, k} \in V_{N}[u p]$. (En effet si $\nu_{N}$ est une fonction de $V_{N}[u p]$ à support minimal et si $\nu_{N}^{*} \in C_{c}^{\infty}$ vérifie

$$
\left\langle\nu_{N}^{*}\left(x-\frac{k}{2^{N}}\right), \nu_{N}(x)\right\rangle=\delta_{\ell, 0}
$$

on utilise le projecteur

$$
P^{N} f=\sum_{\ell}\left\langle f, \nu^{*}\left(x-\frac{\ell}{2^{N}}\right)\right\rangle \nu\left(x-\frac{\ell}{2^{N}}\right)
$$

sur $V_{N}[u p]$. Son noyau est proprement supporté de sorte que $P_{N} \varphi_{N, k}=$ $\varphi_{N, k}$ est immédiat).

Il reste à démontrer la convergence dans $C^{\infty}$ (convergence uniforme sur tout compact de fonctions et de leurs dérivées) vers une fonction de $K^{\rho}$ de

$$
\sum_{N=1}^{\infty} \sum_{k \in \mathbb{Z}} c_{N, k} \varphi_{N, k}
$$

lorsque $\left\{\rho^{-N} 2^{-N(N+1) / 2} c_{N, k}\right\} \in \ell^{\infty}\left(\mathbb{N}^{*} \times \mathbb{Z}\right)$. Pour cela, on va noter $K_{N, p}$ le nombre

$$
K_{N, p}=\sup \left\{\left\|\sum_{k \in \mathbb{Z}} c_{N, k} \frac{d^{p}}{d x^{p}} \varphi_{N, k}\right\|_{\infty}: \sup _{k}\left|c_{N, k}\right| \leq 1\right\} .
$$


Comme $\varphi_{0, k}=u p(x-1+k)$, on a (puisque supp up $\left.=[0,2]\right)$

$$
K_{0, p} \leq 2\left\|\frac{d^{p}}{d x^{p}} u p\right\|_{\infty}=2 \cdot 2^{p(p+1) / 2}
$$

Nous allons alors estimer $K_{N, p}$ par récurrence sur $N$. On commence par remarquer que si $f \in K^{\rho}$ vérifie pour tout $p \geq p_{0}$ et tout $\ell \in \mathbb{Z}$,

$$
\frac{d^{p}}{d x^{p}} f\left(\frac{\ell}{2^{p-1}}\right)=0
$$

alors

$$
f=\sum_{\ell} f(\ell) \varphi_{0, \ell}+\sum_{p=1}^{p_{0}-1} \sum_{\ell} f^{(p)}\left(\frac{f}{2^{p-1}}\right) \varphi_{p, \ell} .
$$

(En effet la convergence de

$$
g=\sum_{\ell} f(\ell) \varphi_{0,2}+\sum_{p=1}^{p_{0}-1} \sum_{\ell} f^{(p)}\left(\frac{\ell}{2^{p-1}}\right) \varphi_{p, \ell}
$$

est immédiate, la série étant localement finie. Par ailleurs, l'appartenance de $g$ à $K^{\rho}$ est immédiate: il suffit de remarquer que $\varphi_{p, \ell}(x+1)=$ $\varphi_{p, \ell-2^{p-1}}(x)$, de sorte que les estimations de taille sur les dérivées de $g$ sont immédiatement uniformes. Enfin $f=g$ puisque $\left.S_{\rho}(f)=S_{\rho}(g)\right)$. On remarque également que si $f \in K^{\rho}$ alors $(d f / d x)(x / 2) \in K^{\rho}$ : si $g=(d f / d x)(x / 2)$ on a

$$
\begin{aligned}
\left\|\frac{d^{N}}{d x^{N}} g\right\|_{\infty} & =2^{-N}\left\|\frac{d^{N+1}}{d x^{N+1}} f\right\|_{\infty} \\
& \leq 2^{-N} C \rho^{N+1} 2^{(N+1)(N+2) / 2} \\
& =2 C \rho \rho^{N} 2^{N(N+1) / 2}
\end{aligned}
$$

On note alors $\varepsilon_{N}=\sum_{k \in \mathbb{Z}} c_{N, k} \varphi_{N, k}$. Si $N \geq 2$, on a

$$
\begin{aligned}
\frac{d \varepsilon_{N}}{d x}= & \frac{1}{2^{N}} \sum_{k \in \mathbb{Z}} c_{N, k} 2 \varphi_{N-1, k}(2 x) \\
& +\sum_{k} \frac{1}{2} \frac{d \varepsilon_{N}}{d x}\left(\frac{2 k+1}{2}\right) 2 u p(2 x-2 k) .
\end{aligned}
$$


Cela nous donne

$$
\begin{aligned}
\left\|\frac{d^{N} \varepsilon_{N}}{d x^{N}}\right\|_{\infty} \leq & \left\|\frac{d^{N-1}}{d x^{N-1}} \sum_{k \in \mathbb{Z}} c_{N, k} \varphi_{N-1, k}\right\|_{\infty} \\
& +2^{N(N+1) / 2} \sup _{k}\left|\frac{1}{2} \frac{d \varepsilon_{N}}{d x}\left(\frac{2 k+1}{2}\right)\right| .
\end{aligned}
$$

Par ailleurs on a

$$
\begin{aligned}
\varepsilon_{N}\left(k_{0}+1\right)-\varepsilon_{N}\left(k_{0}\right)= & 0 \\
= & \int_{k_{0}}^{k_{0}+1} \frac{d \varepsilon_{N}}{d x} d x \\
= & \frac{1}{2} \frac{d \varepsilon_{N}}{d x}\left(\frac{2 k_{0}+1}{2}\right) \\
& +\int_{k_{0}}^{k_{0}+1} \frac{1}{2^{N}} \sum_{k \in \mathbb{Z}} c_{N, k} 2 \varphi_{N-1, k}(2 x) d x,
\end{aligned}
$$

ce qui donne

$$
\left|\frac{1}{2} \frac{d \varepsilon_{N}}{d x}\left(\frac{2 k_{0}+1}{2}\right)\right| \leq \frac{2}{2^{N}}\left\|\sum_{k \in \mathbb{Z}} c_{N, k} \varphi_{N-1, k}\right\|_{\infty} .
$$

Le Lemme 8 (lemme de majoration) permet alors d'écrire

$$
\left\|\sum_{k \in \mathbb{Z}} c_{N, k} \varphi_{N-1, k}\right\|_{\infty} \leq 2^{3-N-N(N-1) / 2}\left\|\frac{d^{N-1}}{d x^{N-1}} \sum_{k} c_{N, k} \varphi_{N-1, k}\right\|_{\infty}
$$

Au total, on a donc obtenu

$$
\begin{aligned}
K_{N, N} & \leq K_{N-1, N-1}\left(1+2^{N(N+1) / 2} \frac{2}{2^{N}} 2^{3-N-N(N-1) / 2}\right) \\
& =K_{N-1, N-1}\left(1+\frac{16}{2^{N}}\right) .
\end{aligned}
$$

On obtient donc

$$
K_{N, N} \leq \prod_{j=2}^{N}\left(1+\frac{16}{2^{j}}\right) K_{1,1} \leq C_{0}=\prod_{j=2}^{\infty}\left(1+\frac{16}{2^{j}}\right) K_{1,1}
$$


Par ailleurs $K_{0,0} \leq 1$ et $K_{1,1}$ est bien fini puisque

$$
K_{1,1} \leq\left\|\frac{d \varphi_{1,0}}{d x}\right\|_{\infty}+\left\|\frac{d \varphi_{1,1}}{d x}\right\|_{\infty}+\left\|\frac{d \varphi_{1,2}}{d x}\right\|_{\infty}
$$

On a donc montré

$$
\sup _{N \in \mathbb{N}} K_{N, N} \leq \prod_{j=2}^{\infty}\left(1+\frac{16}{2^{j}}\right) \cdot K_{1,1}=C_{0} .
$$

L'estimation de $K_{N, p}$ est alors facile:

- si $p<N$, on note

$$
\Omega_{N, p}=2^{N(N+1) / 2} 2^{-p(p+1) / 2} K_{N, p}
$$

et en utilisant à nouveau (27) on obtient

$$
K_{N, p} \leq 2^{p-N} K_{N-1, p-1}+2^{p(p+1) / 2} \frac{16}{2^{N}} 2^{-N(N+1) / 2} K_{N-1, N-1}
$$

et donc

$$
\Omega_{N, p} \leq \Omega_{N-1, p-1}+\frac{16}{2^{N}} C_{0} \leq \Omega_{N-p, 0}+16 C_{0} \sum_{N-p+1}^{N} \frac{1}{2^{r}}
$$

Par ailleurs, le Lemme 8 (lemme de majoration) donne que

$$
K_{N-p, 0} \leq K_{N-p, N-p} 42^{-(N-p)-(N-p)(N-p+1) / 2}
$$

et donc $\Omega_{N-p, 0} \leq 42^{-(N-p)} C_{0}$. Au total

(29) $K_{N, p} \leq 2^{-N(N+1) / 2} 2^{p(p+1) / 2} 2^{-(N-p)} 5 C_{0}, \quad$ pour $0 \leq p \leq N$.

- Si $p>N$, on remarque que

$\frac{d^{N}}{d x^{N}}\left(\sum c_{N, k} \varphi_{N, k}\right)=\sum_{\ell \in \mathbb{Z}}\left(\frac{d^{N}}{d x^{N}} \sum c_{N, k} \varphi_{N, k}\right)\left(\frac{\ell}{2^{N}}\right) u p\left(2^{N} x+1-\ell\right)$

et donc (en utilisant que $u p(x)$ et $u p(x+2)$ sont à supports disjoints)

$$
K_{N, p} \leq 2 K_{N, N} 2^{-N(N+1) / 2} 2^{p(p+1) / 2}, \quad \text { pour } p \geq N .
$$


On conclut alors que si $\rho^{-N} 2^{-N(N+1) / 2}\left|c_{N, k}\right| \leq M$, on a

$$
\begin{aligned}
\left\|\frac{d^{p}}{d x^{p}} \sum_{N} \sum_{k} c_{N, k} \varphi_{N, k}\right\|_{\infty} \leq & \sum_{N=1}^{\infty} K_{N, p} M \rho^{N} 2^{N(N+1) / 2} \\
\leq & M \sum_{N<p} 2 C_{0} 2^{p(p+1) / 2} \rho^{N} \\
& +M \sum_{p \leq N} 5 C_{0} 2^{p(p+1) / 2} \rho^{N} 2^{-(N-p)} \\
\leq & C_{0} M \rho^{p} 2^{p(p+1) / 2}\left(2 \sum_{\ell \leq 0} \rho^{-\ell}+5 \sum_{\ell \geq 0}\left(\frac{\rho}{2}\right)^{\ell}\right) .
\end{aligned}
$$

La Proposition 5 et donc démontrée.

Nous allons maintenant décrire l'analyse multi-résolution non-stationnaire engendrée par up (décrite dans [11] par N. Dyn et A. Ron).

Proposition 6. a) Soit up $p_{k}$ la fonction de support minimum dans $V_{k}[u p]$ normalisée par inf supp up $p_{k}=0$ et $\widehat{u p}_{k}(0)=1$. Alors on a

$$
\begin{gathered}
\widehat{u p}_{k}\left(2^{k} \xi\right)=\left(\frac{1-e^{-i \xi}}{i \xi}\right)^{k} \widehat{u p}(\xi) \\
\widehat{u p}_{k}\left(2^{k} \xi\right)=\prod_{j=1}^{\infty} m_{j+k}\left(\frac{\xi}{2^{j}}\right) \quad \text { avec } m_{j}(\xi)=\left(\frac{1+e^{-i \xi}}{2}\right)^{j}
\end{gathered}
$$

En particulier, up engendre une analyse multirésolution non-stationnaire normale.

b) Soit $\Pi_{k}$ le projecteur orthogonal de $L^{2}$ sur $V_{k}[u p]$. Alors pour tout $N \geq 0$, il existe une constante $C_{N}>0$ telle que, pour tous $k \geq N$, $f \in H^{N}$

$$
\left\|f-\Pi_{k} f\right\|_{2} \leq C_{N} 2^{-k N}\left\|f^{(N)}\right\|_{2} .
$$

En particulier, la fonction up a un ordre d'approximation infini.

Preuve. a) On a

$$
\widehat{u p}(\xi)=\prod_{j=0}^{\infty} \hat{\chi}\left(\frac{\xi}{2^{j}}\right)
$$


Par ailleurs, on sait que

$$
\hat{\chi}(\xi)=\prod_{j=1}^{\infty}\left(\frac{1+e^{-i \xi / 2^{j}}}{2}\right) .
$$

On obtient donc

$$
\widehat{u p}(\xi)=\prod_{j=0}^{\infty}\left(\prod_{\ell=1}^{\infty} \frac{1+e^{-i \xi / 2^{j+\ell}}}{2}\right)=\prod_{\ell=1}^{\infty}\left(\frac{1+e^{-i \xi / 2^{\ell}}}{2}\right)^{\ell},
$$

Le regroupement des termes étant autorisé par le fait que si $|\xi| \leq \pi$,

$$
\sum_{j=0}^{+\infty} \sum_{\ell=1}^{\infty}\left|\log \frac{1+e^{-i \xi / 2^{j+\ell}}}{2}\right|<+\infty
$$

En particulier,

$$
\widehat{u p}(\xi)=\prod_{j=1}^{k} m_{j}\left(\frac{\xi}{2^{j}}\right) \hat{\gamma}_{k}(\xi)
$$

avec

$$
\hat{\gamma}_{k}=\prod_{j=k+1}^{\infty} m_{j}\left(\frac{\xi}{2^{j}}\right) .
$$

On vérifie facilement que $\gamma_{k} \in V_{k}[u p] \cap L_{\text {comp }}^{2}$. (Il suffit de vérifier que si $\varphi \in L_{\text {comp }}^{2}$ vérifie $\sum(-1)^{k} \varphi(x-k)=0$, alors $\gamma$ défini par

$$
\hat{\gamma}(\xi)=\hat{\varphi}(\xi) \frac{2}{1+e^{-i \xi}}
$$

vérifie $\gamma \in V_{0}[\varphi] \cap L_{\text {comp }}^{2}$ : en fait on a $\varphi=(\gamma(x)+\gamma(x+1)) / 2$, ou encore

$$
\gamma=2 \sum_{0}^{\infty} \varphi(x+k)=-2 \sum_{-\infty}^{-1} \varphi(x+k) .
$$

On a alors $\gamma \in L_{\mathrm{loc}}^{2}$, supp $\gamma$ compact, donc $\gamma \in L_{\text {comp }}^{2}$ et $\left.\gamma \in V_{0}[\varphi]\right)$. De plus $\gamma_{k}$ est de support minimal dans $V_{k}[u p]$ : on peut utiliser le critère d'A. Ron [24] qui dit que $\nu$ est le support minimum dans $V_{0}[\mu]$ si la fonction entière $\hat{\nu}(z)=\int \nu(x) e^{-i z x} d x$ n'a pas de zéro (complexe) $2 \pi$ périodique. Ce critère est évident: si $\nu$ n'est pas de support minimum,

$$
\nu=\sum_{k_{0}}^{k_{1}} a_{k} \nu_{0}(x-k) \quad \text { et } \quad \hat{\nu}(z)=\left(\sum_{k_{0}}^{k_{1}} a_{k} e^{-i k z}\right) \hat{\nu}_{0}(z),
$$


si $\nu$ par contre est de support minimum et si $\omega \in C_{c}^{\infty}$ vérifie $\langle\omega, \nu(x-$ $k)\rangle=\delta_{k, 0}$ alors

$$
\sum_{k \in \mathbb{Z}} \hat{\nu}(z+2 k \pi) \hat{\omega}(z+2 k \pi)
$$

converge uniformément sur tout compact de $\mathbb{C}$ vers la fonction 1 .

Nous avons donc prouvé (32), et donc que $\left\{V_{k}[u p]\right\}_{k \geq 0}$ est normale. (31) se déduit immédiatement de $(32)$ en mettant $\left(1+e^{-i \xi} / 2\right)^{k}$ en facteur dans $m_{j+k}(\xi)$.

b) l'estimation (33) est relativement immédiate. On écrit $f=$ $f_{k}+g_{k}$ où

$$
\hat{f}_{k}=\chi_{[-\pi, \pi]}\left(\frac{\xi}{2^{k}}\right) \hat{f}
$$

On a

$$
\left\|g_{k}-\Pi_{k} g_{k}\right\|_{2} \leq 2\left\|g_{k}\right\|_{2} \leq 2\left(2^{k} \pi\right)^{-N}\left\|f^{(N)}\right\|_{2}
$$

Par ailleurs, on a

$$
\begin{aligned}
\left\|\Pi_{k} f_{k}\right\|_{2}^{2} & =\frac{1}{2 \pi} \int_{-\infty}^{+\infty} \frac{\left|\sum_{\ell \in \mathbb{Z}} \hat{f}_{k}\left(\xi+\ell 2 \pi 2^{k}\right) \widehat{u p}_{k}\left(\xi+\ell 2 \pi 2^{k}\right)\right|^{2}}{\left(\sum_{\ell \in \mathbb{Z}}\left|\widehat{u p}_{k}\left(\xi+2 \pi \ell 2^{k}\right)\right|^{2}\right)^{2}}\left|\widehat{u p}_{k}(\xi)\right|^{2} d \xi \\
& =\frac{1}{2 \pi} \int_{-\pi 2^{k}}^{\pi 2^{k}}\left|\hat{f}_{k}(\xi)\right|^{2} \frac{\left|\widehat{u p}_{k}(\xi)\right|^{2}}{\sum_{\ell \in \mathbb{Z}}\left|\widehat{u p}_{k}\left(\xi+2 \pi \ell 2^{k}\right)\right|^{2}} d \xi
\end{aligned}
$$

et donc

$$
\begin{aligned}
\left\|f_{k}-\Pi_{k} f_{k}\right\|_{2}^{2} & =\left\|f_{k}\right\|_{2}^{2}-\left\|\Pi_{k} f_{k}\right\|_{2}^{2} \\
& =\frac{1}{2 \pi} \int_{-\pi 2^{k}}^{\pi 2^{k}}\left|\hat{f}_{k}(\xi)\right|^{2} \frac{\sum_{\ell \neq 0}\left|\widehat{u p}_{k}\left(\xi+2 \pi \ell 2^{k}\right)\right|^{2}}{\sum_{\ell \in \mathbb{Z}}\left|\widehat{u p}_{k}\left(\xi+2 \pi \ell 2^{k}\right)\right|^{2}} d \xi
\end{aligned}
$$

On écrit

$$
\widehat{u p}_{k}(\xi)=\left(\frac{1-e^{-i \xi / 2^{k}}}{i \xi / 2^{k}}\right)^{k} \widehat{u p}\left(\frac{\xi}{2^{k}}\right),
$$


d'où

$$
\begin{aligned}
\frac{\sum_{\ell \neq 0}\left|\widehat{u p}_{k}\left(\xi+2 \pi \ell 2^{k}\right)\right|^{2}}{\sum_{\ell}\left|\widehat{u p}_{k}\left(\xi+2 \pi \ell 2^{k}\right)\right|^{2}} & \leq \sum_{\ell \neq 0} \frac{\left|\widehat{u p}_{k}\left(\xi+2 \pi \ell 2^{k}\right)\right|^{2}}{\left|\widehat{u p}_{k}(\xi)\right|^{2}} \\
& =\sum_{\ell \neq 0}\left|\frac{\xi}{\xi+2 \pi \ell 2^{k}}\right|^{2 k} \frac{\left|\widehat{u p}\left(\xi / 2^{k}+2 \pi \ell\right)\right|^{2}}{\left|\widehat{u p}\left(\xi / 2^{k}\right)\right|^{2}}
\end{aligned}
$$

Si $|\xi| \leq \pi 2^{k}$, alors

$$
\frac{1}{\left|\widehat{u p}\left(\xi / 2^{k}\right)\right|} \leq \sup _{|\eta| \leq \pi} \frac{1}{|\widehat{u p}(\eta)|}<+\infty
$$

tandis que

$$
\frac{|\xi|}{\left|\xi+2 \pi \ell 2^{k}\right|} \leq \frac{|\xi|}{2 \pi 2^{k}-|\xi|} \leq 1
$$

et

$$
\frac{|\xi|}{\left|\xi+2 \pi \ell 2^{k}\right|} \leq \frac{|\xi|}{2 \pi 2^{k}-|\xi|} \leq \frac{|\xi|}{\pi 2^{k}} .
$$

On obtient donc pour $k \geq N$,

$$
\begin{aligned}
& \left\|f_{k}-\Pi_{k} f\right\|_{2}^{2} \leq \frac{1}{2 \pi} \int_{-\pi 2^{k}}^{\pi 2^{k}}|\hat{f}(\xi)|^{2}\left(\frac{|\xi|}{\pi 2^{k}}\right)^{2 N} \\
& \cdot \frac{\sup _{|\eta| \leq \pi} \sum_{\ell \neq 0}|\widehat{u p}(\eta+2 \pi \ell)|^{2}}{\inf _{|\eta| \leq \pi}|\widehat{u p}(\eta)|^{2}} d \xi \\
& \leq C_{N} 2^{-2 k N}\left\|f^{(N)}\right\|_{2}^{2} .
\end{aligned}
$$

La Proposition 6 est donc démontrée.

En fait, non seulement l'approximation par up et ses translatées est spectrale, mais elle est de plus optimale. Plus précisément, Rvachev a étudié l'approximation des fonctions $2 \pi$-périodiques par des translatées (périodisées) de $u p(x / \pi)$. Rappelons que si $B$ est un espace de Banach et si $A$ est une partie de $B$, le diamètre de Kolmogorov $d_{n}(A, B)$ est défini par

$$
d_{n}(A, B)=\inf _{\substack{V_{n} \subset B \\ \operatorname{dim} V_{n}=n}} \sup _{a \in A} \inf _{v \in V_{n}}\|a-v\|_{B}
$$


C'est la mesure de la meilleure approximation possible de $A$ par des vecteurs choisis dans un sous-espace de dimension $n$. On notera $E_{B}$ $(A, V)$ la quantité $\sup _{a \in A} \inf _{v \in V}\|a-v\|_{B}$.

Les $2 \pi$-périodisées de $u p\left(\left(x-1 / 2^{k}\right) / \pi\right)$ forment un espace de dimension $2^{k+1}$, noté $U p_{k}$. Rvachev montre alors [26]:

- si $B=L^{2}$ et $A_{N}=\left\{f \in H^{N}(\mathbb{R} / 2 \pi \mathbb{Z}):\left\|f^{(N)}\right\|_{2} \leq 1\right\}$, il existe $k_{0}(N)$ tel que pour $k \geq k_{0}(N), E_{L^{2}}\left(A_{N}, U p_{k}\right)=d_{2^{k+1}}\left(A_{N}, L^{2}\right)$.

- Si $B=L^{\infty}$ et $\tilde{A}_{N}=\left\{f \in C^{N-1}(\mathbb{R} / 2 \pi \mathbb{Z}): f^{(N)} \in L^{\infty}\right.$ et $\left.\left\|f^{(N)}\right\|_{\infty} \leq 1\right\}$, alors

$$
\lim _{k \rightarrow+\infty} \frac{E_{L^{\infty}}\left(\tilde{A}_{N}, U p_{k}\right)}{d_{2^{k+1}}\left(\tilde{A}_{N}, L^{\infty}\right)}=1 .
$$

Comme le souligne Rvachev, les approximations optimales pour ces diamètres de Kolmogorov sont réalisées par les polynômes trigonométriques ou par les fonctions splines. Cependant les polynômes trigonométriques n'offrent pas de bonnes propriétés de localisation spatiale (puisque leur support est $\mathbb{R} / 2 \pi \mathbb{Z}$ tout entier) tandis que les splines ont un ordre d'approximation fini. La fonction de Rvachev combine les deux aspects (support compact, approximation spectrale) tout en étant optimale (pour l'approximation de $H^{N}$ ) ou asymptotiquement optimale (pour l'approximation de $C^{N}$ ).

On peut aussi étudier l'interpolation par des translatées de up. Plus précisément, Rvachev montre que $V_{N}[u p]$ contient une interpolante $\Lambda_{N}$ telle que

$$
\delta_{k, 0}= \begin{cases}\Lambda_{N}\left(\frac{k}{2^{N}}\right), & \text { si } N \text { est pair } \\ \Lambda_{N}\left(\frac{2 k+1}{2^{N+1}}\right), & \text { si } N \text { est impair } .\end{cases}
$$

Il s'intéresse alors à l'opérateur d'interpolation

$$
I_{N} f= \begin{cases}\sum_{k} f\left(\frac{2 k \pi}{2^{N}}\right) \Lambda_{N}\left(\frac{x}{\pi}-\frac{k}{2^{N}}\right), & \text { si } N \text { est pair } \\ \sum_{k} f\left(\frac{2 k+1}{2^{N+1}} 2 \pi\right) \Lambda_{N}\left(\frac{x}{\pi}-\frac{k}{2^{N}}\right), & \text { si } N \text { est impair }\end{cases}
$$

Il montre que l'on a, si $f \in C^{M}(\mathbb{R} / 2 \pi \mathbb{Z})$,

$$
\left\|f-I_{N} f\right\|_{\infty} \leq C_{M} \frac{1+\log N}{2^{N M}}\left\|f^{(M)}\right\|_{\infty}
$$


(alors que l'interpolation aux $2^{N+1}$ points $2 k \pi / 2^{N}, 0 \leq k<2^{N}$, par un polynôme trigonométrique ne donne une qualité d'approximation que de l'ordre de $N\left\|f^{(M)}\right\|_{\infty} / 2^{N M}$ [31]; on passe donc d'une estimation d'erreur

$$
\frac{2^{N M}\left\|f-I_{N} f\right\|_{\infty}}{\left\|f^{(M)}\right\|_{\infty}}
$$

de l'ordre de $N=\log _{2} 2^{N}$ à l'ordre de $\log N=\log \log _{2} 2^{N}$ ).

\section{La base de Berkolaïko et Novikov.}

En 1992, V. Berkolaïko et I. Novikov ont introduit une modification de la fonction de Rvachev pour obtenir une base orthonormale de $L^{2}(\mathbb{R})$ composée d'ondelettes non stationnaires $C^{\infty}$ à support compact [2]. Cette construction a été également introduite par A. Cohen et N. Dyn en 1993 [7], dans le cadre des travaux de N. Dyn sur les analyses multirésolutions non stationnaires. L'idée est de remplacer dans la formule (32) le filtre

$$
m_{j}(\xi)=\left(\frac{1+e^{-i \xi}}{2}\right)^{j}
$$

(qui est le fitre d'échelle du $B$-spline de degré $j-1$ ) par le filtre de Daubechies

$$
m_{j}(\xi)=\left(\frac{1+e^{-i \xi}}{2}\right)^{j} \mu_{j}(\xi)
$$

où $\mu_{j}=\sum_{k=0}^{j-1} \mu_{j, k} e^{-i k \xi}$ est tel que $\left|m_{j}(\xi)\right|^{2}+\left|m_{j}(\xi+\pi)\right|^{2}=1$. En effet, ce filtre conduit à une fonction d'échelle qui a le même ordre d'approximation que le spline de degré $j-1$ mais qui de plus engendre une base orthonormée d'ondelettes à support compact.

Proposition 7. Soient $\left\{m_{N}\right\}_{N \geq 1}$ des filtres de Daubechies, avec

$$
m_{N}(\xi)=\left(\frac{1+e^{-i \xi}}{2}\right)^{N} Q_{N}\left(e^{-i \xi}\right)
$$

ò̀ $Q_{N} \in \mathbb{R}[X], \operatorname{deg} Q_{N}=N-1, Q_{N}(1)=1$ et

$$
\left|m_{N}(\xi)\right|^{2}+\left|m_{N}(\xi+\pi)\right|^{2}=1
$$


On définit alors pour $N \geq 0, \Phi_{N}$ et $\Psi_{N}$ par les formules

$$
\begin{gathered}
\hat{\Phi}_{N}(\xi)=\prod_{j=1}^{\infty} m_{N+j}(\xi) \\
\hat{\Psi}_{N}(\xi)=e^{-i \xi / 2} \bar{m}_{N+1}\left(\frac{\xi}{2}+\pi\right) \hat{\Phi}_{N+1}\left(\frac{\xi}{2}\right) .
\end{gathered}
$$

Alors

i) $\Phi_{N}$ et $\Psi_{N}$ sont $C^{\infty} \grave{a}$ support compact, $\operatorname{supp} \Phi_{N} \subset[0,2 N+3]$, $\operatorname{supp} \Psi_{N} \subset[-N, N+2]$.

ii) La famille $\left\{2^{N / 2} \Phi_{N}\left(2^{N} x-k\right)\right\}_{k \in \mathbb{Z}}$ est une base orthonormée de $V_{N}\left[\Phi_{0}\right]$. (En particulier $\Phi_{N}\left(2^{N} x\right)$ est de support minimal dans $V_{N}\left[\Phi_{0}\right]$ et $\Phi_{0}$ engendre une analyse multi-résolution non-stationnaire normale).

iii) La famille $\left\{2^{N / 2} \Psi_{N}\left(2^{N} x-k\right)\right\}_{k \in \mathbb{Z}}$ est une base orthonormée de $V_{N+1}\left[\Phi_{0}\right] \cap V_{N}\left[\Phi_{0}\right]^{\perp}$. En particulier, la famille $\left\{\Phi_{0}(x-k)\right\}_{k \in \mathbb{Z}} \cup$ $\left\{2^{N / 2} \Psi_{N}\left(2^{N} x-k\right)\right\}_{N \geq 0, k \in \mathbb{Z}}$ est une base orthonormée de $L^{2}(\mathbb{R})$.

iv) $\Phi_{0}$ a un ordre d'approximation infini. Plus précisément, pour tout $s \in \mathbb{R}$ et tout $f \in \mathcal{D}^{\prime}(\mathbb{R}), f$ appartient à l'espace de Sobolev $H^{s}(\mathbb{R})$ si et seulement si $N_{s}(f)<+\infty$ où

$N_{s}(f)=\left(\sum_{k \in \mathbb{Z}}\left|\left\langle f, \Phi_{0}(x-k)\right\rangle\right|^{2}+\sum_{N=0}^{+\infty} \sum_{k \in \mathbb{Z}} 4^{N s}\left|\left\langle f, 2^{N / 2} \Psi_{N}\left(2^{N} x-k\right)\right\rangle\right|^{2}\right)^{1 / 2}$

et les normes $\|f\|_{H^{s}}$ et $N_{s}(f)$ sont équivalentes.

Preuve. La convergence du produit infini (34) est immédiate. En effet, nous avons $\left\|m_{N}\right\|_{\infty} \leq 1$ et $\operatorname{deg} m_{N}=2 N-1$, de sorte que l'inégalité de Bernstein nous donne $\left\|d m_{N} / d \xi\right\|_{\infty} \leq C N$, et donc

$$
\left|m_{N+j}\left(\frac{\xi}{2^{j}}\right)-1\right| \leq C \frac{N+j}{2^{j}}|\xi|,
$$

comme

$$
\sum_{j=1}^{\infty}(N+j) \frac{1}{2^{j}}=N+2<+\infty,
$$

le produit converge. Par ailleurs, chaque produit fini est borné par 1; la convergence a donc lieu dans $\mathcal{S}^{\prime}$. Par la transformation de Fourier inverse, on voit que $\Phi_{N}$ est un produit infini de convolution de sommes 
de masses de Dirac; le $j$-ième terme du produit de convolution a son support contenu dans $\left[0,(2 N+2 j-1) / 2^{j}\right]$, de sorte que $\operatorname{supp} \Phi_{N} \subset$ $[0,2 N+3]$.

Par ailleurs, puisque $\left|m_{N}(\xi)\right|^{2}+\left|m_{N}(\xi+\pi)\right|^{2}=1$ pour tous $N$ et $\xi$, les fonctions $\theta_{N, p}$ définies par

$$
\hat{\theta}_{N, p}(\xi)=\chi_{[-\pi, \pi]}\left(\frac{\xi}{2^{p}}\right) \prod_{j=1}^{p} m_{N+j}\left(\frac{\xi}{2^{j}}\right)
$$

engendrent des familles orthogonales $\left\{\theta_{N, p}(x-k)\right\}_{k \in \mathbb{Z}}$. Comme $\hat{\theta}_{N, p} \rightarrow$ $\hat{\Phi}_{N}$ quand $p \rightarrow+\infty$ (la convergence étant ponctuelle), la preuve de la Proposition 7 se réduit aux estimations suivantes

$$
\begin{gathered}
\quad\left|\hat{\theta}_{N, p}(\xi)\right| \leq C_{1}|\xi|^{-\alpha|\log \xi|}, \\
\left|\hat{\Psi}_{N}(\xi)\right| \leq D_{k}|\xi|^{k}, \quad \text { pour } k \in \mathbb{N}, \quad k \leq N+1,
\end{gathered}
$$

où $C_{1}$ et $\alpha$ sont des constantes positives ne dépendant ni de $N$ ni de $p$, et où $D_{k}$ ne dépend pas de $N$.

(37) est évident si $|\xi| \leq 2^{30} 2 \pi$ : on écrit $\left|\hat{\theta}_{N, p}(\xi)\right| \leq 1$. Si $|\xi| \geq$ $2^{30} 2 \pi$, on note $\ell$ l'entier $(\geq 30)$ tel que $2^{\ell} 2 \pi \leq|\xi|<2^{\ell+1} 2 \pi$. Si $p \leq \ell$, $\hat{\theta}_{N, p}(\xi)=0$. Si $p \geq \ell+1$, on a

$$
\left|\hat{\theta}_{N, p}(\xi)\right| \leq \prod_{j=1}^{\ell+1}\left|m_{N+j}\left(\frac{\xi}{2^{j}}\right)\right|=A_{N, \ell}(\xi) B_{N, \ell}(\xi)
$$

où

$$
A_{N, \ell}(\xi)=\prod_{j=1}^{\ell+1}\left|\frac{1+e^{-i \xi / 2^{j}}}{2}\right|^{N+j} \quad \text { et } \quad B_{N, \ell}(\xi)=\sum_{j=1}^{\ell+1}\left|Q_{N+j}\left(e^{-i \xi / 2^{j}}\right)\right| .
$$

$A_{N, \ell}$ se majore facilement du fait que pour $|\xi| \leq 2^{\ell+1} \pi,\left|\sin \left(\xi / 2^{\ell+2}\right)\right| \geq$ $(2 / \pi)\left(|\xi| / 2^{\ell+2}\right)$,

$$
\begin{aligned}
A_{N, \ell}(\xi) & =\prod_{j=1}^{\ell+1}\left|\frac{1+e^{-i \xi / 2^{j}}}{2}\right|^{N} \prod_{k=0}^{\ell}\left|\prod_{j=k+1}^{\ell+1} \frac{1+e^{-i \xi / 2^{j}}}{2}\right| \\
& =\left|\frac{\sin (\xi / 2)}{2^{\ell+1} \sin \left(\xi / 2^{\ell+2}\right)}\right|^{N} \prod_{k=0}^{\ell}\left|\frac{\sin \left(\xi / 2^{k+1}\right)}{2^{\ell+1-k} \sin \left(\xi / 2^{\ell+2}\right)}\right| \\
& \leq\left(\frac{\pi}{|\xi|}\right)^{N} \prod_{k=0}^{\ell} \frac{2^{k} \pi}{|\xi|} \\
& \leq 2^{-\ell N} 2^{-\ell(\ell+1) / 2} .
\end{aligned}
$$


Pour $B_{N, \ell}$, on part de la formule de Daubechies pour $\left|Q_{N}\left(e^{-i \xi}\right)\right|[8]$

$$
\left|Q_{N}\left(e^{-i \xi}\right)\right|^{2}=\sum_{k=0}^{N-1} C_{N+k-1}^{k}\left(\frac{1-\cos \xi}{2}\right)^{k} .
$$

Berkolaïko et Novikov ont remarqué que, puisque $C_{N+k-1}^{k} \leq C_{N+k}^{k}$ si $k \leq N-1$, on a $\left|Q_{N}\left(e^{-i \xi}\right)\right| \leq\left|Q_{N+1}\left(e^{-i \xi}\right)\right|$ pour tous $N$ et $\xi$. Par ailleurs, le contrôle de $Q_{N}\left(e^{-i \xi}\right) Q_{N}\left(e^{-2 i \xi}\right)$ est classique

$$
\left|Q_{N}\left(e^{-i \xi}\right) Q_{N}\left(e^{-2 i \xi}\right)\right| \leq\left|Q_{N}(-1) Q_{N}\left(e^{-2 i \pi / 3}\right)\right| \leq 2^{N-1 / 2} 3^{N / 2} .
$$

En effet,

$$
A_{N}(X)=\sum_{k=0}^{N-1} C_{k+N-1}^{k}\left(\frac{1-X}{2}\right)^{k}
$$

décroît sur $[-1,1]$; de plus on a toujours $\cos \xi \geq-1 / 2$ ou $\cos 2 \xi \geq-1 / 2$, de sorte que

$$
\left|Q_{N}\left(e^{-i \xi}\right) Q_{N}\left(e^{-2 i \xi}\right)\right| \leq\left|Q_{N}(-1) Q_{N}\left(e^{-2 i \pi / 3}\right)\right|
$$

Par ailleurs, si $X<0$, on a

$$
A_{N}(X) \leq 2^{2 N-2}\left(\frac{1-X}{2}\right)^{N-1}\left(\frac{1-X}{-X}\right)
$$

il suffit d'écrire que $C_{N+k-1}^{k} \leq C_{N+k}^{k+1} / 2$ donc que

$$
C_{N+k-1}^{k} \leq 2^{k-N+1} C_{2 N-2}^{N-1} \leq 2^{2 N-2} 2^{k-N+1},
$$

cela donne $\left|Q_{N}(-1)\right| \leq 2^{N-1 / 2}$ et $\left|Q_{N}\left(e^{-2 i \pi / 3}\right)\right| \leq 3^{N / 2}$. Cette estimation sur $A_{N}(X)$ donne également lorsque $\cos \xi<0$,

$$
\left|m_{N}(\xi)\right| \leq\left(\frac{1+\cos \xi}{2}\right)^{N / 2}(1-\cos \xi)^{N / 2} 2^{N-1 / 2} \frac{1}{\sqrt{-\cos \xi}}=\frac{|\sin \xi|^{N}}{\sqrt{2|\cos \xi|}}
$$

L'estimation de $B_{N, \ell}$ est alors facile. En effet, si $\ell=2 q$, on écrit

$$
\begin{aligned}
\left|B_{N, \ell}(\xi)\right| & \leq\left|Q_{N+1}\left(e^{-i \xi / 2}\right) \prod_{r=1}^{q} Q_{2 r+1+N}\left(e^{-i \xi / 2^{2 r}}\right) Q_{2 r+1+N}\left(e^{-i \xi / 2^{2 r+1}}\right)\right| \\
& \leq 2^{N+1 / 2} \prod_{r=1}^{q} 2^{N+2 r+1 / 2} 3^{N / 2+r+1 / 2} \\
& =(2 \sqrt{3})^{N \ell / 2+\ell(\ell+2) / 4} 2^{N+1 / 2} 6^{\ell / 4}
\end{aligned}
$$


tandis que si $\ell=2 q-1$ on écrit

$$
\begin{aligned}
\left|B_{N, \ell}(\xi)\right| & \leq \prod_{r=1}^{q}\left|Q_{N+2 r}\left(e^{-i \xi / 2^{2 r-1}}\right) Q_{N+2 r}\left(e^{-i \xi / 2^{2 r}}\right)\right| \\
& \leq \prod_{r=1}^{q} 2^{N+2 r-1 / 2} 3^{N / 2+r} \\
& =(2 \sqrt{3})^{N(\ell+1) / 2+(\ell+1)(\ell+3) / 4} 2^{-(\ell+1) / 2} .
\end{aligned}
$$

On obtient dans tous les cas

$$
\left|B_{N, \ell}(\xi)\right| \leq(2 \sqrt{3})^{N(\ell+2) / 2}(2 \sqrt{3})^{(\ell+2)(\ell+3) / 4}
$$

et donc

$$
\left|A_{N, \ell} B_{N, \ell}\right| \leq 2^{-\ell N}(2 \sqrt{3})^{N(\ell+2) / 2}(2 \sqrt{3})^{(\ell+2)(\ell+3) / 4} 2^{-\ell(\ell+1) / 2} .
$$

Comme $\ell \geq 30,(\ell+2) / 2 \leq 8 \ell / 15$ et donc

$$
\left|A_{N, \ell} B_{N, \ell}\right| \leq\left(\frac{(2 \sqrt{3})^{8 / 15}}{2}\right)^{\ell N}\left(\frac{\sqrt{3}}{2}\right)^{\ell^{2} / 4}\left(3^{5 / 8} 2^{3 / 4}\right)^{\ell}(2 \sqrt{3})^{3 / 2} .
$$

Comme $\gamma=(2 \sqrt{3})^{8 / 15} / 2$ est $<1\left(\operatorname{car} \gamma^{30}=3^{8} 2^{16} / 2^{30}=3^{8} / 2^{14}=\right.$ $\left.(81 / 128)^{2}\right)$ et $\sqrt{3} / 2<1$, on a en choisissant $\alpha<\log (\sqrt{3} / 2) / 4$ (et $\alpha>0)$

$$
\left|A_{N, \ell} B_{N, \ell}(\xi)\right| \leq C \gamma^{\ell N} e^{-\alpha \ell^{2}},
$$

où $C$ ne dépend ni de $N$, ni de $\ell$. Comme $\ell \geq \log (|\xi| / 2 \pi) / \log 2$, on a

$$
\left|\hat{\theta}_{N}(\xi)\right| \leq C\left(\frac{|\xi|}{2 \pi}\right)^{-N \log (1 / \gamma) / \log 2}\left(\frac{|\xi|}{2 \pi}\right)^{-\alpha \log (|\xi| / 2 \pi) /(\log 2)^{2}}
$$

(37) est donc démontré.

(38) est immédiat. En effet, si $|\xi| \geq 1$, on a $|\hat{\Psi}(\xi)| \leq 1$. Si $|\xi| \leq 1$, on a $|\xi / 2+\pi| \in[2 \pi / 3,4 \pi / 3]$ (d'où $\cos (\xi+\pi) \leq-1 / 2$ ); on écrit alors

$$
\left|\hat{\Psi}_{N}(\xi)\right| \leq\left|m_{N+1}\left(\frac{\xi}{2}+\pi\right)\right| \leq \frac{|\sin (\xi / 2)|^{N+1}}{\sqrt{2 \cos (\xi / 2)}} \leq\left(\frac{|\xi|}{2}\right)^{N+1} \leq\left(\frac{|\xi|}{2}\right)^{k}
$$

La Proposition 7 est alors facile à établir. i) et ii) sont immédiats. Le point iii) est facile à vérifier: si $f, g \in V_{N}\left[\Phi_{0}\right]$,

$$
\hat{f}(\xi)=F\left(\frac{\xi}{2^{N}}\right) \hat{\Phi}_{N}\left(\frac{\xi}{2^{N}}\right)
$$


et

$$
\hat{g}(\xi)=G\left(\frac{\xi}{2^{N}}\right) \hat{\Phi}_{N}\left(\frac{\xi}{2^{N}}\right)
$$

on a alors

$$
\langle f, g\rangle=2^{N} \int_{-\pi}^{\pi} F(\xi) \bar{G}(\xi) \frac{d \xi}{2 \pi} .
$$

On applique ceci à $f=\Psi_{N-1}$ et à $g=\Phi_{N-1}(x-k)$ ou $\Psi_{N-1}(x-k)$.

Enfin, la caractérisation de $H^{s}$ est aisée à établir. En utilisant (37) et (38), on vérifie que pour tout $k \in \mathbb{N}$

$$
\begin{aligned}
& \sup _{N \geq 0} \sup _{\xi} \sum_{p \in \mathbb{Z}}|\xi+2 \pi p|^{k}\left|\hat{\Psi}_{N}(\xi+2 \pi p)\right|^{2}<+\infty, \\
& \sup _{N \geq k-1} \sup _{\xi} \sum_{p \in \mathbb{Z}}|\xi+2 \pi p|^{-k}\left|\hat{\Psi}_{N}(\xi+2 \pi p)\right|^{2}<+\infty .
\end{aligned}
$$

Cela entraîne que, si $\Lambda^{s}$ est l'opérateur $\widehat{\Lambda^{s} f}=|\xi|^{s} \hat{f}$, on a pour $s \in \mathbb{R}$ et $N \geq-s$

$$
\left\|\Lambda^{s} Q_{N} f\right\|_{2} \leq C_{s} 2^{N s}\left\|Q_{N} f\right\|_{2}
$$

où

$$
Q_{N} f=\sum_{k} 2^{N}\left\langle f, \Psi_{N}\left(2^{N} x-k\right)\right\rangle \Psi_{N}\left(2^{N} x-k\right) .
$$

En particulier, on a

$$
\begin{aligned}
\left|\left\langle\Lambda^{s} Q_{N} f, \Lambda^{s} Q_{N^{\prime}} f\right\rangle\right| & =\left|\left\langle\Lambda^{s \pm 1} Q_{N} f, \Lambda^{s \mp 1} Q_{N^{\prime}} f\right\rangle\right| \\
& \leq 2^{N s} 2^{N^{\prime} s} 2^{-\left|N-N^{\prime}\right|}\left\|Q_{N} f\right\|_{2}\left\|Q_{N^{\prime}} f\right\|_{2}
\end{aligned}
$$

et cela entraîne

$$
\|f\|_{H^{s}} \leq C\left\|N_{s} f\right\|_{2} \simeq C\left(\left\|P_{0} f\right\|_{2}^{2}+\sum_{0}^{\infty} 4^{N s}\left\|Q_{N} f\right\|_{2}^{2}\right)^{1 / 2}
$$

(où $P_{0}$ est le projecteur orthogonal sur $V_{0}\left[\Phi_{0}\right]$ ). L'inégalité inverse s'obtient alors par dualité.

REMARQUe. On obtient facilement que

$$
\lim _{N \rightarrow+\infty}\left|\hat{\Phi}_{N}(\xi)\right|= \begin{cases}1, & \text { si }|\xi|<\pi, \\ \frac{1}{\sqrt{2}}, & \text { si }|\xi|=\pi, \\ 0, & \text { si }|\xi|>\pi .\end{cases}
$$


En particulier, par le théorème d'Ascoli, cela entraîne que

$$
\lim _{N \rightarrow+\infty} \inf _{x_{0}} \int\left|x-x_{0}\right|^{2}\left|\Phi_{N}(x)\right|^{2} d x=+\infty .
$$

Ainsi, le support numérique de $\Phi_{N}$ tend vers l'infini. Cela entraîne des difficultés pour décrire les propriétés d'analyse fonctionnelle de la base de Berkolaïko et Novikov.

Dans les sections suivantes, nous allons décrire des analyses multirésolutions non-stationnaires où les fonctions d'échelle non-stationnaires auront un bon comportement global en espace et en fréquence.

\section{Analyse multi-résolution quasi-stationnaire.}

Le manque de contrôle du comportement asymptotique des fonctions $\Phi_{N}$ de Berkolaïko et Novikov provient de ce que les filtres de Daubechies ont un mauvais comportement asymptotique lorsque $N \rightarrow$ $+\infty$

$$
\lim _{N \rightarrow+\infty}\left|m_{N}(\xi)\right|= \begin{cases}1, & \text { si }|\xi|<\frac{\pi}{2}, \\ \frac{1}{\sqrt{2},} & \text { si }|\xi|=\frac{\pi}{2}, \\ 0, & \text { si } \frac{\pi}{2}<|\xi| \leq \pi .\end{cases}
$$

La situation estdifférente lorsque les filtres convergent dans $C^{\infty}(\mathbb{R} / 2 \pi \mathbb{Z})$

Definition. Une analyse multi-résolution quasi-stationnaire est l'analyse multi-résolution non-stationnaire $\left\{V_{k}\left[\Phi_{0}\right]\right\}_{k \geq 0}$ associée à une fonction $\Phi_{0}$ qui vérifie

i) $\hat{\Phi}_{0}(\xi)=\prod_{j=1}^{\infty} m_{j}\left(\xi / 2^{j}\right)$.

ii) $m_{j}$ est un polynôme trigonométrique à coefficients réels tel que

$$
\sum_{j=1}^{\infty} \frac{1}{2^{j}} \operatorname{deg} m_{j}<+\infty .
$$

iii) $m_{j}$ converge dans $C^{\infty}(\mathbb{R} / 2 \pi \mathbb{Z})$ vers une fonction $m_{\infty}$ qui est un filtre d'échelle associé à une fonction d'échelle régulière $\varphi_{\infty}$.

iv) Pour $j$ assez grand, $m_{j}(\pi)=0$ et $m_{j}(0)=1$. 
En fait, seules les propriétés de $m_{\infty}$ déterminent les propriétés de l'analyse multi-résolution.

Théorème 2 (Deuxième théorème d'approximation). Soient $\left\{m_{j}\right\}_{j \geq 1}$ des fonctions de $C^{\infty}(\mathbb{R} / 2 \pi \mathbb{Z})$ vérifiant les hypothèses suivantes:

i) $m_{j}$ converge dans $C^{\infty}(\mathbb{R} / 2 \pi \mathbb{Z})$ vers le filtre d'échelle $m_{\infty}$ d'une fonction d'échelle régulière $\varphi_{\infty}$.

ii) Pour $j$ assez grand, $m_{j}(0)=1$ et $m_{j}(\pi)=0$.

Alors

a) Les fonctions $\hat{\Phi}_{j}(\xi)=\prod_{\ell=1}^{\infty} m_{j+\ell}\left(\xi / 2^{\ell}\right), j \geq 0$, sont $C^{\infty}$ et de carré intégrable. De plus leurs transformées de Fourier inverses $\Phi_{j}$ sont $\grave{a}$ décroissance rapide dans $L^{2}$ et convergent rapidement vers $\varphi_{\infty}$ dans $L^{2}$.

b) De même si $\alpha>0$, si $\varphi_{\infty} \in H^{\alpha+\varepsilon}$ pour un $\varepsilon>0$ et si pour $j$ assez grand on $a\left(\partial^{p} / \partial \xi^{p}\right) m_{j}(\pi)=0$ pour $0 \leq p \leq[\alpha]$, les $\Phi_{j}$ sont $\grave{a}$ décroissance rapide dans $H^{\alpha}$ et convergent rapidement vers $\varphi_{\infty}$ dans $H^{\alpha}$.

c) Si tous les $m_{j}$ sont à valeurs positives ou nulles, si $\alpha>0$, si $\varphi_{\infty} \in$ $B_{\infty}^{\alpha+\varepsilon, \infty}$ pour un $\varepsilon>0$ et si pour $j$ assez grand on a $\left(\partial^{p} / \partial \xi^{p}\right) m_{j}(\pi)$ $=0$ pour $0 \leq p \leq 2[\alpha / 2]+1$, alors les $\Phi_{j}$ sont à décroissance rapide dans $B_{\infty}^{\alpha, \infty}$ et convergent rapidement vers $\varphi_{\infty}$ dans $B_{\infty}^{\alpha, \infty}$.

d) Tous les $m_{j}$ vérifient $\left|m_{j}(\xi)\right|^{2}+\left|m_{j}(\xi+\pi)\right|^{2}=1$ si et seulement si toutes les familles $\left\{\Phi_{j}(x-k)\right\}_{k \in \mathbb{Z}}$ sont des familles orthonormales, (i.e. $\left.\left\langle\Phi_{j}(x-k), \Phi_{j}(x-\ell)\right\rangle=\delta_{k, \ell}\right)$.

e) Si tous les $m_{j}$ sont à valeurs positives ou nulles, alors tous les $m_{j}$ vérifient $m_{j}(\xi)+m_{j}(\xi+\pi)=1$ si et seulement si toutes les fonctions $\Phi_{j}$ sont interpolantes, $\left(\right.$ i.e. $\left.\Phi_{j}(k)=\delta_{k, 0}\right)$.

f) Si tous les $m_{j}$ sont des polynômes trigonométriques et si

$$
\sum_{j=1}^{\infty}\left(\frac{\operatorname{deg} m_{j}}{2^{j}}\right)<+\infty
$$

alors toutes les $\Phi_{j}$ sont à support compact et pour $j$ assez grand $\left\{\Phi_{j}\left(2^{j} x\right.\right.$ $-k)\}_{k \in \mathbb{Z}}$ est une base de Riesz de $V_{j}\left[\Phi_{0}\right]$.

Remarques. a) Parmi les propriétés qui ne peuvent pas se lire sur $m_{\infty}$, on peut se demander dans f) si $\Phi_{j}(2 x)$ est de support minimum 
dans $V_{j}\left[\Phi_{0}\right]$. C'est bien sûr le cas si $\Phi_{j}$ est interpolante (cas e)) ou orthogonale ( $\operatorname{cas} \mathrm{d})$ ).

b) Contrairement au cas de la fonction up [11], on a un contrôle de

$$
\frac{\sup _{\left\|\lambda_{k}\right\|_{2}=1}\left\|\sum_{k} \lambda_{k} \Phi_{j}(x-k)\right\|_{2}}{\inf _{\left\|\lambda_{k}\right\|_{2}=1}\left\|\sum_{k} \lambda_{k} \Phi_{j}(x-k)\right\|_{2}},
$$

uniforme en $j$ (pour $j$ assez grand): en effet, cette quantité est donnée par

$$
\frac{\sup _{\xi}\left(\sum_{k}\left|\hat{\Phi}_{j}(\xi+2 k \pi)\right|^{2}\right)^{1 / 2}}{\inf _{\xi}\left(\sum_{k}\left|\hat{\Phi}_{j}(\xi+2 k \pi)\right|^{2}\right)^{1 / 2}}
$$

et on montre facilement que $\sum_{k}\left|\hat{\Phi}_{j}(\xi+2 k \pi)\right|^{2}$ converge uniformément $\operatorname{vers} \sum_{k}\left|\hat{\varphi}_{\infty}(\xi+2 k \pi)\right|^{2}$.

Preuve. La preuve de a), b), c) est similaire à celle du Théorème 1, demandant juste un peu de précautions oratoires pour tenir compte de la non-stationarité.

On vérifie facilement que $\hat{\Phi}_{j}$ est $C^{\infty}$. En effet, si $V$ est un voisinage compact de $\xi_{0} \in \mathbb{R}$, on a sur $V, \operatorname{Re} m_{j+\ell}\left(\xi / 2^{\ell}\right) \geq 1 / 2$ pour $\ell$ assez grand; il suffit de remarquer que

$$
\left|m_{N}(\xi)-m_{N}(0)\right| \leq \sup _{k}\left\|\frac{d}{d \xi} m_{k}\right\|_{\infty}|\xi| .
$$

On a alors

$$
\left|\log m_{j+\ell}\left(\frac{\xi}{2^{\ell}}\right)\right| \leq 2 \sup _{k}\left\|\frac{d}{d \xi} m_{k}\right\|_{\infty} \frac{|\xi|}{2^{\ell}}
$$

et pour $p \geq 1$

$$
\left|\frac{d^{p}}{d \xi^{p}} \log m_{j+\ell}\left(\frac{\xi}{2^{\ell}}\right)\right| \leq\left(\frac{1}{2}\right)^{\ell_{p}} 4^{p} p !\left(\sum_{q=0}^{p} \sup _{k}\left\|\frac{d^{q}}{d \xi^{q}} m_{k}\right\|_{\infty}\right)^{p} .
$$

Cela assure que $\sum_{\ell \geq \ell_{0}} \log m_{j+\ell}\left(\xi / 2^{\ell}\right)$ est $C^{\infty}$ sur $\stackrel{\circ}{V}$, et il en va de même pour

$$
\hat{\Phi}_{j}=\prod_{1 \leq \ell<\ell_{0}} m_{j+\ell}\left(\frac{\xi}{2^{\ell}}\right) e^{\sum_{\ell \geq \ell_{0}} \log m_{j+\ell}\left(\xi / 2^{\ell}\right)} .
$$


Cette démonstration permet également de voir qu'il existe $C$ et $M \geq 0$ tels que pour tout $j$ et tout $\xi$ on ait: $\left|\hat{\Phi}_{j}(\xi)\right| \leq C(1+|\xi|)^{M}$. Par convergence dominée, cela entraîne la convergence de $\Phi_{j}$ vers $\varphi_{\infty}$ dans $H^{s}$ pour $s<-M-1 / 2$ et dans $B_{\infty}^{\delta, \infty}$ pour $\delta<-M-1$. Pour terminer la démonstration de a), b), c), il suffit de vérifier que dans le cas b) on a, pour $0<\varepsilon^{\prime}<\min \{\varepsilon,[\alpha]+1-\alpha\}$ pour tout $k \in \mathbb{N}$,

$$
\sup _{j}\left\|x^{k} \Phi_{j}\right\|_{H^{\alpha+\varepsilon^{\prime}}}<+\infty
$$

et dans le cas c) on a, pour $0<\varepsilon^{\prime}<\min \{\varepsilon, 2[\alpha / 2]+2-\alpha\}$ pour tout $k \in \mathbb{N}$,

$$
\sup _{j}\left\|x^{k} \Phi_{j}\right\|_{B_{\infty}^{\alpha+\varepsilon^{\prime}, \infty}}<+\infty .
$$

Bien évidemment, on peut se restreindre à $j \geq j_{0}$ et écrire:

- dans le cas b),

$$
m_{j}(\xi)=\left(\frac{1+e^{-i \xi}}{2}\right)^{N} \mu_{j}(\xi)
$$

$\left(j \geq j_{0}\right.$ ou $\left.j=+\infty\right)$ où $N=[\alpha]+1 ;$ on a $\mu_{j} \rightarrow \mu_{\infty}$ dans $C^{\infty}(\mathbb{R} / 2 \pi \mathbb{Z})$ et si

$$
\hat{\Omega}_{j}=\prod_{k=1}^{\infty} \mu_{j+k}\left(\frac{\xi}{2^{\ell}}\right),
$$

on a

$$
\hat{\Phi}_{j}=\left(\frac{1-e^{-i \xi}}{i \xi}\right)^{N} \hat{\Omega}_{j}
$$

et le problème est alors de montrer que

$$
\sup _{j}\left\|x^{k} \Omega_{j}\right\|_{H^{\alpha-N+\varepsilon^{\prime}}}<+\infty
$$

- dans le cas c),

$$
m_{j}(\xi)=\left(\frac{1+\cos \xi}{2}\right)^{N} \mu_{j}(\xi)
$$

où $N=2[\alpha / 2]+2$ et

$$
\hat{\Phi}_{j}=\left(\frac{2(1-\cos \xi)}{\xi^{2}}\right)^{N} \hat{\Omega}_{j}
$$


et le problème est de montrer que

$$
\sup _{k}\left\|x^{k} \Omega_{j}\right\|_{B_{\infty}^{\alpha-2 N+\varepsilon^{\prime}, \infty}}<+\infty .
$$

Nous traitons le cas b), le cas c) se traitant de manière totalement analogue (en étudiant la norme $\left\|x^{k} \Omega_{j}\right\|_{B_{1}^{\alpha-2 N+\varepsilon^{\prime \prime}, \infty}}$ ). Nous cherchons à estimer

$$
I_{j, k}=\int_{|\xi| \geq \pi}|\xi|^{2 \alpha+2 \varepsilon^{\prime}-2 N}\left|\frac{\partial^{k}}{\partial \xi^{k}} \hat{\Omega}_{j}(\xi)\right|^{2} d \xi,
$$

ou encore (puisque $\alpha+\varepsilon^{\prime}-N<0$ )

$$
\begin{aligned}
\tilde{I}_{j, k} & =\sum_{\ell=0}^{\infty} \int_{|\xi| \leq 2^{\ell} 2 \pi}(1+|\xi|)^{2 \alpha+2 \varepsilon^{\prime}-2 N}\left|\frac{\partial^{k}}{\partial \xi^{k}} \hat{\Omega}_{j}(\xi)\right|^{2} d \xi \\
& \leq C \sum_{\ell=0}^{\infty}\left(2^{\ell}\right)^{2 \alpha+2 \varepsilon^{\prime}-2 N} \sup _{\xi} \sum_{|\xi+2 \pi p| \leq 2^{\ell} 2 \pi}\left|\frac{\partial^{k}}{\partial \xi^{k}} \hat{\Omega}_{j}(\xi+2 \pi p)\right|^{2}
\end{aligned}
$$

On note alors

$$
M_{j, k, \ell}(\xi)=\sum_{|\xi+2 \pi p| \leq 2^{\ell} 2 \pi}\left|\frac{\partial^{k}}{\partial \xi^{k}} \hat{\Omega}_{j}(\xi+2 \pi p)\right|^{2} .
$$

Si $k=0$, on a

$$
\begin{aligned}
M_{j, 0, \ell}(\xi) & =\left|\mu_{j+1}\left(\frac{\xi}{2}\right)\right|^{2} M_{j+1,0, \ell-1}\left(\frac{\xi}{2}\right)+\left|\mu_{j+1}\left(\frac{\xi}{2}+\pi\right)\right|^{2} M_{j+1,0, \ell-1}\left(\frac{\xi}{2}\right) \\
& =T_{j+1} \circ T_{j+2} \circ \cdots \circ T_{j+\ell}\left(M_{j+\ell, 0,0}\right)
\end{aligned}
$$

où $T_{j}$ est l'opérateur de transition associé à $\mu_{j}$, i.e. $T_{j}$ est défini par

$$
T_{j} f=\left|\mu_{j}\left(\frac{\xi}{2}\right)\right|^{2} f\left(\frac{\xi}{2}\right)+\left|\mu_{j}\left(\frac{\xi}{2}+\pi\right)\right|^{2} f\left(\frac{\xi}{2}+\pi\right) .
$$

Par positivité des opérateurs $T_{j}$ et contrôle uniforme des $\hat{\Omega}_{j}$ sur $[-2 \pi$, $2 \pi$ ], on obtient

$$
\left|M_{j, 0, \ell}(\xi)\right| \leq C T_{j+1} \circ T_{j+2} \circ \cdots \circ T_{j+\ell}(1)(\xi)
$$

Par ailleurs, si $\rho$ est choisi de façon à ce que $4^{N-\alpha-\varepsilon}<\rho<4^{N-\alpha-\varepsilon^{\prime}}$, on sait qu'il existe $Q$ tel que $\left\|T_{\infty}^{Q}(1)\right\|_{\infty} \leq \rho^{Q}$; si maintenant $\rho^{\prime}$ est choisi 
de façon à ce que $\rho<\rho^{\prime}<4^{N-\alpha-\varepsilon^{\prime}}$, on aura qu'il existe $j_{1}$ tel que si $j \geq j_{1}$ on a

$$
\left\|T_{j+1} \circ T_{j+2} \circ \cdots \circ T_{j+Q}(1)\right\|_{\infty} \leq \rho^{\prime Q}
$$

et cela entraîne, pour tous $j$ et $\ell$,

$$
\left\|M_{j, 0, \ell}\right\|_{\infty} \leq C \rho^{\ell \ell}
$$

et donc $\tilde{I}_{j, 0} \leq C \sum_{0}^{\infty}\left(\rho^{4^{\alpha+\varepsilon^{\prime}-N}}\right)^{\ell}<+\infty$.

On traite de même $M_{j, k, \ell}$ : on a

$$
\frac{\partial^{k}}{\partial \xi^{k}} \hat{\Omega}_{j}=\frac{1}{2^{k}} \sum_{\ell=0}^{k} C_{k}^{\ell} \frac{\partial^{\ell}}{\partial \xi^{\ell}} \hat{\Omega}_{j+1}\left(\frac{\xi}{2}\right) \frac{\partial^{k-\ell}}{\partial \xi^{k-\ell}} \hat{\mu}_{j+1}\left(\frac{\xi}{2}\right)
$$

d'où

$$
\begin{aligned}
& \left|M_{j, k, \ell}(\xi)\right| \\
& \leq \frac{1}{2^{2 K-1}} T_{j+1}\left(M_{j+1, k, \ell-1}\right)(\xi) \\
& \quad+2 k \sum_{q=1}^{k} \frac{1}{4^{k}}\left(C_{k}^{q}\right)^{2}\left(\left|m_{j+1}^{(q)}\left(\frac{\xi}{2}\right)\right|^{2} M_{j+1, k-q, \ell-1}\left(\frac{\xi}{2}\right)\right. \\
& \left.\quad+\left|m_{j+1}^{(q)}\left(\frac{\xi}{2}+\pi\right)\right|^{2} M_{j+1, k-q, \ell-1}\left(\frac{\xi}{2}+\pi\right)\right) .
\end{aligned}
$$

Par récurrence, on suppose montré que $\left|M_{N, q, L}(\xi)\right| \leq C \rho^{L}$ pour $q<k$ où $C, \rho$ ne dépendent ni de $N$ ni de $L$ et $\max \left\{1,4^{N-\alpha-\varepsilon}\right\}<\rho<4^{N-\alpha}$. On a alors

$$
\left|M_{j, k, \ell}(\xi)\right| \leq \frac{1}{2^{2 k-1}} T_{j+1}\left(M_{j+1, k, \ell-1}\right)(\xi)+C \rho^{\ell-1},
$$

ce qui donne

$$
\begin{aligned}
M_{j, k, \ell}(\xi) \leq & \left(\frac{1}{2^{2 k-1}}\right)^{\ell} T_{j+1} \circ T_{j+2} \circ \cdots \circ T_{j+\ell}\left(M_{j+\ell, k, 0}\right) \\
& +C \sum_{q=0}^{\ell-1} T_{j+1} \circ \cdots \circ T_{j+q}(1) \frac{\rho^{\ell-q-1}}{\left(2^{2 k-1}\right)^{q}} .
\end{aligned}
$$

Or nous savons montrer que pour tous $j$ et $\ell$ on a

$$
\left\|T_{j+1} \circ T_{j+2} \circ \cdots \circ T_{j+\ell}(1)\right\|_{\infty} \leq C \rho^{\ell}
$$


et par ailleurs $\sup _{j}\left\|M_{j, k, 0}\right\|_{\infty}<+\infty$, de sorte que

$$
\left\|M_{j, k, \ell}\right\|_{\infty} \leq C\left(\frac{1}{2^{2 k-1}}\right)^{\ell}+\rho^{\ell-1} \sum_{q=0}^{\ell-1} \frac{1}{\left(2^{2 k-1}\right)^{q}} \leq C^{\prime} \rho^{\ell} .
$$

Nous avons donc prouvé

$$
\sup _{j}\left\|x^{k} \Phi_{j}\right\|_{H^{\alpha+\varepsilon}}<+\infty
$$

Les points d) et e) sont relativement évidents. Démontrons par exemple d). Si $\Phi_{j}$ et $\Phi_{j+1}$ sont orthonormales (au sens que les familles $\left\{\Phi_{j}(x-\right.$ $k)\}_{k \in \mathbb{Z}}$ et $\left\{\Phi_{j+1}(x-k)\right\}_{k \in \mathbb{Z}}$ le sont), alors $m_{j+1}$ vérifie

$$
\begin{aligned}
1= & \sum_{k \in \mathbb{Z}}\left|\hat{\Phi}_{j}(2 \xi+2 k \pi)\right|^{2} \\
= & \left|m_{j+1}(\xi)\right|^{2} \sum_{k \in \mathbb{Z}}\left|\hat{\Phi}_{j+1}(\xi+2 k \pi)\right|^{2} \\
& +\left|m_{j+1}(\xi+\pi)\right|^{2} \sum_{k \in \mathbb{Z}}\left|\hat{\Phi}_{j+1}(\xi+\pi+2 k \pi)\right|^{2} \\
= & \left|m_{j+1}(\xi)\right|^{2}+\left|m_{j+1}(\xi+\pi)\right|^{2} .
\end{aligned}
$$

Inversement, supposons que $\left|m_{j}(\xi)\right|^{2}+\left|m_{j}(\xi+\pi)\right|^{2}=1$ pour tout $\xi$; désignons par $K$ un compact d'Albert Cohen associé à $m_{\infty}$; pour tout $p$, la fonction $\theta_{N, p}$, définie par

$$
\hat{\theta}_{N, p}(\xi)=\prod_{j=1}^{p} m_{N+j}\left(\frac{\xi}{2^{j}}\right) \chi_{K}\left(\frac{\xi}{2^{p}}\right)
$$

vérifie

$$
\left\langle\theta_{N, p}(x), \theta_{N, p}(x-k)\right\rangle=\delta_{k, 0}
$$

par ailleurs, on a

$$
\left|\hat{\theta}_{N, p}(\xi)\right| \leq\left|\hat{\Phi}_{N}(\xi)\right| \frac{1}{\inf _{\eta \in K}\left|\hat{\Phi}_{N+p}(\eta)\right|},
$$

comme $\hat{\Phi}_{N+p}$ converge uniformément vers $\hat{\varphi}_{\infty}$ sur $K$ et que $\inf _{K}\left|\hat{\varphi}_{\infty}\right|>$ 0 , on peut appliquer la convergence dominée: $\theta_{N, p} \rightarrow \Phi_{N}$ dans $L^{2}$ quand $p \rightarrow+\infty$, de sorte qu'on a bien

$$
\left\langle\Phi_{N}(x), \Phi_{N}(x-k)\right\rangle=\delta_{k, 0} .
$$


Enfin, le point f) est évident, d'après la remarque qui suit le Théorème 2.

Les théorèmes 1 et 2 donnent deux résultats d'approximation d'une fonction d'échelle par d'autres fonctions d'échelle (stationnaires pour le Théorème 1, non-stationnaires pour le Théorème 2). La section suivante décrit de telles approximations.

\section{Polynômes de Bernstein, fonctions d'échelle interpolantes et ondelettes de Kharkov.}

Le point ii) de la Proposition 3 (qui caractérise les filtres des fonctions d'échelle interpolantes (à transformée de Fourier positive)) et le point e) du Théorème 2 (qui caractérise les filtres associés à une suite quasi-stationnaire de fonctions d'échelle interpolantes nonstationnaires) ont ramené l'étude de ces filtres à celle des fonctions $m_{0}$ vérifiant:

i) $m_{0} \in C^{\infty}(\mathbb{R} / 2 \pi \mathbb{Z}), m_{0}(\xi)=\overline{m_{0}(-\xi)}$,

ii) $m_{0}(0)=1$ et $m_{0}(\xi) \geq 0$ pour tout $\xi$,

iii) $m_{0}(\xi)+m_{0}(\xi+\pi)=1$ pour tout $\xi$,

iv) $m_{0}$ vérifie le critère d'Albert Cohen.

Il est alors facile de vérifier que les conditions i) à iii) équivalent à $m_{0}(\xi)=F((1+\cos \xi) / 2)$ où

j) $F \in C^{\infty}([0,1])$,

jj) $F(1)=1$ et $F(t) \geq 0$ pour $t \in[0,1]$,

jjj) $F(t)+F(1-t)=1$ pour tout $t \in[0,1]$.

Le cas où $m_{0}$ est un polynôme trigonométrique est particulièrement simple: la condition jjj) s'écrit pour $F$, si $\operatorname{deg} m_{0} \leq N$,

$$
F(t)=\sum_{k=0}^{N} \varepsilon_{N, k} C_{N}^{k} t^{k}(1-t)^{N-k}, \quad \text { avec } \varepsilon_{N, k}+\varepsilon_{N, N-k}=1
$$

C'est-à-dire que $F$ se représente particulièrement facilement dans la base des polynômes de Bernstein de degré $N$ [1], [18], [29]. 
Notation. Nous noterons $P_{N}$ la classe des polynômes $F$ de degré $\leq N$ vérifiant $F(t)+F(1-t)=1$ (ou encore qui admettent une décomposition (39) dans la base des polynômes de Bernstein avec $\left.\varepsilon_{N, k}=\varepsilon_{N, N-k}\right)$. Nous noterons $P_{N}^{+}$la classe des éléments de $P_{N}$ pour lesquels on a de plus $\varepsilon_{N, 0}=0$ et pour $1 \leq k \leq N, 0 \leq \varepsilon_{N, k} \leq 1$.

Remarquons que si $m_{0}(\xi)=F((1+\cos \xi) / 2)$ avec $F \in P_{N}^{+}$alors $m_{0}$ vérifie automatiquement les propriétés i) à iv). (Le critère d'Albert Cohen est automatiquement vérifié puisque dans ce cas $m_{0}$ ne s'annule qu'en $\pi$ ). La famille $P_{N}^{+}$fournit donc une classe de filtres d'échelle associés à des fonctions d'échelle interpolantes à support compact (contenu dans $[-N, N])$.

De plus, si $m_{0}$ vérifie les propriétés i) à iv), on peut écrire grâce au lemme de Riesz $m_{0}(\xi)=\left|m_{1}(\xi)\right|^{2}$ où $m_{1}$ est un polynôme trigonométrique (à coefficients réels) associé à une fonction d'échelle orthogonale à support compact $\varphi_{1}$ (où "orthogonale" signifie que la famille $\left\{\varphi_{1}(x-\right.$ $k)\}_{k \in \mathbb{Z}}$ est orthonormale). Ainsi, $P_{N}^{+}$est associée à une classe de filtres d'échelle orthogonaux.

Parmi les filtres d'échelle orthogonaux, les plus connus sont les filtres de Daubechies $m_{1}(\xi)=\mu_{N}(\xi)$, définis [8] par $\operatorname{deg} \mu_{N} \leq 2 N+1$, $\left|\mu_{N}(\xi)\right|^{2}$ vérifie i) à iv) et $\mu_{N}$ se factorise par $\left(\left(1+e^{-i \xi}\right) / 2\right)^{\bar{N}+1}$. On a alors

$$
\left|\mu_{N}(\xi)\right|^{2}=F_{N}\left(\frac{1+\cos \xi}{2}\right)
$$

où $F_{N} \in P_{2 N+1}^{+}$: plus précisément on a

$$
F_{N}(t)=\sum_{k=N+1}^{2 N+1} C_{2 N+1}^{k} t^{k}(1-t)^{2 N+1-k}
$$

C'est-à-dire que

$$
\varepsilon_{2 N+1, k}= \begin{cases}1, & \text { si } k>\frac{1}{2}(2 N+1), \\ 0, & \text { si } k<\frac{1}{2}(2 N+1) .\end{cases}
$$

$F_{N}$ peut se caractériser de plusieurs façons. Nous venons de l'introduire comme le seul élément de $P_{2 N+1}$ qui a un zéro d'ordre au moins $N+1$ en 0 . C'est aussi le seul polynôme de degré $2 N+1$ qui vérifie $F(t)=$ $O\left(t^{N+1}\right)$ et $F(1+t)=1+O\left(t^{N+1}\right)$; cette description correspond aux filtres maximalement plats de Herrmann [13]. 
Une autre caractérisation est liée à la régularité des fonctions d'échelle associées:

Proposition 8. Pour F vérifiant les propriétés j), jj), jjj) et le critère d'Albert Cohen pour

$$
m_{0}(\xi)=F\left(\frac{1+\cos \xi}{2}\right)
$$

on note $\beta(F)$ le nombre $\beta(F)=\sup \left\{\alpha>0:|\xi|^{\alpha} \hat{\varphi} \in L^{\infty}\right\}$ où $\hat{\varphi}$ est donnée par

$$
\hat{\varphi}(\xi)=\prod_{j=1}^{\infty} m_{0}\left(\frac{\xi}{2^{j}}\right) .
$$

Alors:

i) $F \in P_{2 N+1}^{+}$vérifie $\beta(F)=\max _{G \in P_{2 N+1}^{+}} \beta(G)$ si et seulement si $F=F_{N}$.

ii) Pour tout $\mu \in] 1 / 4,1 / 2\left[\right.$ il existe $\lambda>0$ et $N_{0}>N$ tel que pour $N>N_{0}$ si $F \in P_{N}^{+}$et vérifie $\varepsilon_{N, k}=0$ pour $0 \leq k \leq \mu N$, on a $\beta(F) \geq \lambda N$.

Preuve. i) est presque évident. On remplace $\beta(F)$ par

$$
\beta_{0}(F)=\sup \left\{\alpha>0:\left\{2^{k \alpha} \hat{\varphi}\left(2^{k} \frac{2 \pi}{3}\right)\right\}_{k \geq 0} \in \ell^{\infty}(\mathbb{N})\right\}
$$

Il est clair que $\beta(F) \leq \beta_{0}(F)$; par ailleurs

$$
\left|\hat{\varphi}\left(2^{k} \frac{2 \pi}{3}\right)\right|=\left|\hat{\varphi}\left(\frac{2 \pi}{3}\right)\right| F\left(\frac{1}{4}\right)^{k}
$$

de sorte que $\beta_{0}(F)=-\log F(1 / 4) / \log 2$; enfin, d'après un résultat de Cohen et Conze $([5]), \beta\left(F_{N}\right)=\beta_{0}\left(F_{N}\right)$.

i) se ramène donc à vérifier: pour $F \in P_{2 N+1}^{+}, F \neq F_{N}$, on a $F(1 / 4)>F_{N}(1 / 4)$. 
Mais ceci est évident,

$$
\begin{aligned}
& F\left(\frac{1}{4}\right)-F_{N}\left(\frac{1}{4}\right)= \sum_{k=0}^{N} \varepsilon_{2 N+1, k} C_{2 N+1}^{k} \\
& \cdot\left(\left(\frac{1}{4}\right)^{k}\left(\frac{3}{4}\right)^{2 N+1-k}-\left(\frac{1}{4}\right)^{2 N+1-k}\left(\frac{3}{4}\right)^{k}\right) \\
&=\sum_{k=0}^{N} \varepsilon_{2 N+1, k} C_{2 N+1}^{k}\left(\frac{1}{4}\right)^{k}\left(\frac{3}{4}\right)^{2 N+1-k} \\
& \cdot\left(1-\left(\frac{1}{3}\right)^{2 N+1-2 k}\right) .
\end{aligned}
$$

On a donc $F(1 / 4)-F_{N}(1 / 4)>0$ si l'un des $\varepsilon_{2 N+1, k}(0 \leq k \leq N)$ est $\geq 0$. Le point ii) est assez simple. On factorise dans $F(t)$ un facteur $t^{[\nu N]}$ où $\nu<\mu$

$$
m_{0}(\xi)=\left(\frac{1+\cos \xi}{2}\right)^{[\nu N]} m_{1}(\xi)
$$

et on obtient alors

$$
\hat{\varphi}(\xi)=\left(\frac{2(1-\cos \xi)}{\xi^{2}}\right)^{[\nu N]} \prod_{j=1}^{\infty} m_{1}\left(\frac{\xi}{2^{j}}\right) .
$$

Le produit infini se contrôle aisément, comme nous l'avons fait déjà plusieurs fois, pour $|\xi| \geq \pi$,

$$
\left|\prod_{j=1}^{\infty} m_{1}\left(\frac{\xi}{2^{j}}\right)\right| \leq \sup _{|\eta| \leq 2 \pi}\left|\prod_{j=1}^{\infty} m_{1}\left(\frac{\eta}{2^{j}}\right)\right|\left(\frac{|\xi|}{\pi}\right)^{\log \left\|m_{1}\right\|_{\infty} / \log 2}
$$

et donc on a

$$
\beta(F) \geq 2[\nu N]-\frac{\log \left\|m_{1}\right\|_{\infty}}{\log 2}
$$

Il faut donc estimer $\left\|m_{1}\right\|_{\infty}=\sup _{0 \leq t \leq 1}\left(F(t) / t^{[\nu N]}\right)$. On écrit

$$
\begin{aligned}
\frac{F(t)}{t^{[\nu N]}} & =\sum_{k=[\mu N]+1}^{N} \varepsilon_{N, k} C_{N}^{k} t^{k-[\nu N]}(1-t)^{N-k} \\
& \leq \sum_{k=[\mu N]+1}^{N} C_{N}^{k} t^{k-[\nu N]}(1-t)^{N-k}
\end{aligned}
$$




$$
\begin{aligned}
& \leq \sum_{k=[\mu N]+1}^{N} \frac{C_{N}^{[\nu N]}}{C_{k}^{[\nu N]}} C_{N-[\nu N]}^{k-[\nu N]} t^{k-[\nu N]}(1-t)^{N-k} \\
& \leq \sup _{1+[\mu N] \leq k \leq N} \frac{C_{N}^{[\nu N]}}{C_{k}^{[\nu N]}} \\
& =\frac{C_{N}^{[\nu N]}}{C_{1+[\mu N]}^{[\nu N]}} .
\end{aligned}
$$

On utilise alors la formule de Stirling pour écrire pour $k \geq 1$

$$
\frac{1}{C_{0}}\left(\frac{k}{e}\right)^{k} \sqrt{k} \leq k ! \leq C_{0}\left(\frac{k}{e}\right)^{k} \sqrt{k}
$$

d'où

$$
\begin{aligned}
\left\|m_{1}\right\|_{\infty} & \leq C_{1} \frac{N^{N}}{(N-[\nu N])^{N-[\nu N]}} \frac{(1+[\mu N]-[\nu N])^{1+[\mu N]-[\nu N]}}{(1+[\mu N])^{1+[\mu N]}} \\
& \leq C_{2} \frac{1}{\left(1-\frac{[\nu N]}{N}\right)^{N-[\nu N]}} \frac{\left(\frac{[\mu N]-[\nu N]}{N}\right)^{[\mu N][\nu N]}}{\left(\frac{[\mu N]}{N}\right)^{[\mu N]}} \\
& \leq C_{3}\left(\frac{(\mu-\nu)^{\mu-\nu}}{(1-\nu)^{1-\nu} \mu^{\mu}}\right)^{N}
\end{aligned}
$$

et donc

$$
\beta(F) \geq\left(2 \nu-\frac{1}{\log 2} \log \frac{(\mu-\nu)^{\mu-\nu}}{(1-\nu)^{1-\nu} \mu^{\mu}}\right) N+O(1),
$$

de sorte que ii) est prouvé si on peut choisir $\nu$ tel que

$$
4^{\nu}>\frac{(\mu-\nu)^{\mu-\nu}}{(1-\nu)^{1-\nu} \mu^{\mu}} .
$$

Cela est possible puisque quand $\nu \rightarrow 0^{+}$on a

$$
\begin{aligned}
& 4^{\nu}=1+\nu \log 4+O\left(\nu^{2}\right), \\
\frac{(\mu-\nu)^{\mu-\nu}}{(1-\nu)^{1-\nu} \mu^{\mu}} & =\frac{\left(1-\frac{\nu}{\mu}\right)^{\mu-\nu}}{(1-\nu)^{1-\nu} \mu^{\nu}} \\
& =\frac{\left(1-\nu+O\left(\nu^{2}\right)\right)}{\left(1-\nu+O\left(\nu^{2}\right)\right)\left(1+\nu \log \mu+O\left(\nu^{2}\right)\right)} \\
& =1-\nu \log \mu+O\left(\nu^{2}\right),
\end{aligned}
$$


Comme $4 \mu>1$, on a $\log 4>-\log \mu$ et ii) est démontré.

Remarque. La démonstration du point ii) est basée sur une idée classique ([8]). Cependant si on factorisait tous les zéros $(\nu=\mu)$ comme dans le cas classique, on ne pourrait pas conclure: il faudrait $4^{\mu}>1 /\left((1-\mu)^{1-\mu} \mu^{\mu}\right)$. Mais on a $\mu(1-\mu)<1 / 4$ d'où $(4 \mu(1-\mu))^{\mu}<1$ tandis que $1 /(1-\mu)^{1-2 \mu}>1$. Même dans le cas $\mu=1 / 2$, qui correspond aux filtres de Daubechies, la factorisation totale ne permet pas de conclure $\liminf B\left(F_{N}\right) / N>0$ mais seulement $\liminf \beta\left(F_{N}\right) / \log N>0$. Pour obtenir lim inf $\beta\left(F_{N}\right) / N>0$, il faut alors une étude plus fine de $\prod_{j=1}^{\infty} m_{1}\left(\xi / 2^{j}\right)$.

Bien évidemment, les polynômes de Bernstein peuvent nous permettre d'approximer des fonctions. Nous obtenons alors le théorème suivant:

Théorème 3 (Approximation des fonctions d'échelle interpolantes). Soit $m_{\infty} \in C^{\infty}(\mathbb{R} / 2 \pi \mathbb{Z})$ une fonction qui vérifie

i) $m_{\infty}(\xi)=m_{\infty}(-\xi) \geq 0$ pour tout $\xi$,

ii) $m_{\infty}(0)=1$,

iii) $m_{\infty}(\xi)+m_{\infty}(\xi+\pi)=1$ pour tout $\xi$,

iv) $m_{\infty}$ satisfait le critère d'Albert Cohen,

et soit $\varphi_{\infty}$ la fonction d'échelle interpolante régulière associée à $m_{\infty}$.

Pour $N \geq 1$, on définit $m_{N}$, à l'aide de la fonction $F_{\infty} \in C^{\infty}([0,1])$ définie par

$$
m_{\infty}(\xi)=F_{\infty}\left(\frac{1+\cos \xi}{2}\right),
$$

par la formule suivante

$$
m_{N}(\xi)=\sum_{k=0}^{N} F_{\infty}\left(\frac{k}{N}\right)\left(\frac{1+\cos \xi}{2}\right)^{k}\left(\frac{1-\cos \xi}{2}\right)^{N-k}
$$

Alors:

a) Pour tout $N, m_{N} \in P_{N}^{+}$et donc $m_{N}$ définit une fonction d'échelle interpolante à support compact $\varphi_{N}$.

b) $m_{N} \rightarrow m_{\infty}$ dans $C^{\infty}(\mathbb{R} / 2 \pi \mathbb{Z})$ quand $N \rightarrow+\infty$. 
c) $\varphi_{N}$ converge rapidement vers $\varphi_{\infty}$ dans $L^{\infty}$.

d) Si $\alpha<1$ et si $\varphi_{\infty} \in B_{\infty}^{\alpha+\varepsilon, \infty}$ pour un $\varepsilon>0$, alors il existe $N_{0}$ tel que pour $N>N_{0}, \varphi_{N} \in B_{\infty}^{\alpha, \infty}$ et $\varphi_{N}$ converge rapidement vers $\varphi_{\infty}$ dans $B_{\infty}^{\alpha, \infty}$.

e) Si $m_{\infty}$ est identiquement nulle sur un voisinage de $\pi$, la conclusion $\mathrm{d}$ ) est valable pour tout $\alpha>0$.

f) De même les fonctions d'échelle non stationnaires $\Phi_{N}$, définies par

$$
\hat{\Phi}_{N}(\xi)=\prod_{j=1}^{\infty} m_{N+j}\left(\frac{\xi}{2^{j}}\right),
$$

sont à support compact, interpolantes et convergent rapidement vers $\varphi_{\infty}$ dans $L^{\infty}$, et dans $B_{\infty}^{\alpha, \infty}$ si $\alpha<1$ et $\varphi_{\infty} \in B_{\infty}^{\alpha+\varepsilon, \infty}$ pour un $\varepsilon>0$, et dans $B_{\infty}^{\alpha, \infty}$ si $\alpha>0, \varphi_{\infty} \in B_{\infty}^{\alpha+\varepsilon, \infty}$ pour un $\varepsilon>0$ et $m_{\infty}$ est identiquement nulle sur un voisinage de $\pi$.

Ce Théorème 3 n'est bien sûr qu'un théorème fantôme, il s'agit d'une simple application des théorèmes 1 et 2 et de la théorie des approximations par les polynômes de Bernstein. Rappelons pour mémoire que si $F \in C^{\infty}([0,1])$ les polynômes de Bernstein

$$
\sum_{k=0}^{N} F\left(\frac{k}{N}\right)\left(\frac{1+x}{2}\right)^{k}\left(\frac{1-x}{2}\right)^{N-k}
$$

convergent vers $F$ dans $C^{\infty}([0,1])$.

Cette construction d'interpolante non-stationnaire nous permet alors d'introduire l'interpolante de Kharkov, en hommage à la ville d'où proviennent et les polynômes de Bernstein [3] et la fonction de Rvachev. L'interpolante de Kharkov sera construite à partir d'une interpolante de Lemarié-Meyer [19], désignée également dans la littérature sous le nom d'interpolante de Littlewood-Paley-Meyer: on prend une fonction $F_{\infty} \in C^{\infty}([0,1])$ telle que:

i) $F_{\infty}(t) \geq 0$,

ii) $F_{\infty}(t)+F_{\infty}(1-t)=1$,

iii) $F_{\infty}(t)=0$ pour $|t| \leq 1 / 4$. 
Un exemple de telle fonction peut être définie par

$$
F_{\infty}(t)= \begin{cases}1, & \text { si } t \geq \frac{3}{4} \\ u p\left(2 t-\frac{1}{2}\right), & \text { si } \frac{1}{4} \leq t \leq \frac{3}{4} \\ 0, & \text { si } t \leq \frac{1}{4}\end{cases}
$$

ou encore $(d / d t) F_{\infty}(t)=4 u p(4 t-1)$. La fonction d'échelle $\varphi_{\infty}$ associée à

$$
m_{\infty}=F_{\infty}\left(\frac{1+\cos \xi}{2}\right)
$$

vérifie alors supp $\hat{\varphi}_{\infty} \subset[-4 \pi / 3,4 \pi / 3]$ de sorte que $\varphi_{\infty}$ appartient à la classe de Schwartz $\mathcal{S}(\mathbb{R})$. L'interpolante de Kharkov associée à $F_{\infty}$ est la fonction $\Phi_{0}$ définie par

$$
\hat{\Phi}_{0}(\xi)=\prod_{N=1}^{\infty} m_{N}\left(\frac{\xi}{2^{N}}\right)
$$

où $m_{N}$ est définie par (41).

Théorème 4 (Interpolante de Kharkov). Soit $F_{\infty}$ une interpolante de Lemarié-Meyer (de fonction d'échelle $\left.\varphi_{\infty}\right), \Phi_{0}$ son interpolante de Kharkov et plus généralement $\left\{\Phi_{N}\right\}_{N \geq 0}$ les fonctions d'échelle interpolantes non-stationnaires associées

$$
\left(\hat{\Phi}_{N}(\xi)=\prod_{j=1}^{\infty} m_{N+j}\left(\frac{\xi}{2^{j}}\right)\right)
$$

On désigne par $I_{N}$ l'opérateur d'interpolation

$I_{N}(f)=\sum_{k \in \mathbb{Z}} f\left(\frac{k}{2^{N}}\right) \Phi_{N}\left(2^{N} x-k\right)=\sum_{k \in \mathbb{Z}} 2^{N}\left\langle f, \delta\left(2^{N} x-k\right)\right\rangle \Phi_{N}\left(2^{N} x-k\right)$

et par $\Psi_{N}$ "l'ondelette de Kharkov" $\Psi_{N}=\Phi_{N+1}(2 x-1)$. Alors

i) $\Phi_{N} \in C_{c}^{\infty}$ pour tout $N$ et $\Phi_{N} \rightarrow \varphi_{\infty}$ dans $\mathcal{S}(\mathbb{R})$ quand $N \rightarrow+\infty$.

ii) $I_{N}$ et $I_{N+1}-I_{N}$ sont des projecteurs, 
iii)

$$
\begin{aligned}
\left(I_{N+1}\right. & \left.-I_{N}\right) f \\
& =\sum_{k \in \mathbb{Z}}\left(f\left(\frac{2 k+1}{2^{N+1}}\right)-\sum_{\ell \in \mathbb{Z}} f\left(\frac{\ell}{2^{N}}\right) \Phi_{N}\left(\frac{1}{2}+k-\ell\right)\right) \Psi_{N}\left(2^{N} x-k\right) \\
& =\sum_{k \in \mathbb{Z}} 2^{N}\left\langle f, \theta_{N}\left(2^{N} x-k\right)\right\rangle \Psi_{N}\left(2^{N} x-k\right),
\end{aligned}
$$

où

$$
\theta_{N}=\delta\left(x-\frac{1}{2}\right)-\sum_{\ell \in \mathbb{Z}} \Phi_{N}\left(\frac{1}{2}+\ell\right) \delta(x+\ell) .
$$

iv) Si $p, q \in[1,+\infty]$ et $s>1 / p$ alors pour $f \in \mathcal{D}^{\prime}(\mathbb{R})$ les trois assertions suivantes sont équivalentes:

K1) $f \in B_{q}^{s, p}$,

K2) $I_{0} f \in L^{p}$ et

$$
\left\|2^{N s}\right\|\left(I_{N+1}-I_{N}\right) f\left\|_{L^{p}(\mathbb{R})}\right\|_{\ell^{q}(\mathbb{N})}<+\infty,
$$

K3)

$$
\sum_{k \in \mathbb{Z}}|f(k)|^{p}<+\infty
$$

et

$$
\left\|2^{N(s-1 / p)}\right\| f\left(\frac{2 k+1}{2^{N+1}}\right)-\sum_{\ell} f\left(\frac{\ell}{2^{N}}\right) \Phi_{N}\left(\frac{1}{2}+k-\ell\right)\left\|_{\ell^{p}(k)}\right\|_{\ell^{q}(N)}<+\infty .
$$

Preuve. i) Le point i) est une conséquence directe du Théorème 2.

ii): le fait que $I_{N}$ est un projecteur de $C(\mathbb{R})$ (fonctions continues $\operatorname{sur} \mathbb{R})$ sur

$$
\left\{\sum_{k \in \mathbb{Z}} a_{k} \Phi_{N}\left(2^{N} x-k\right):\left\{a_{k}\right\}_{k \in \mathbb{Z}} \in \mathbb{C}^{\mathbb{Z}}\right\}=V_{N}
$$

est évident. Le fait que $I_{N+1}-I_{N}$ est un projecteur revient à ce que $I_{N+1} \circ I_{N}=I_{N} \circ I_{N+1}=I_{N}$; mais $I_{N+1} \circ I_{N}=I_{N}$ car $V_{N} \subset V_{N+1}$ (par construction de $\Phi_{N}$ ) tandis que $I_{N} \circ I_{N+1}=I_{N}$ est évident puisque $I_{N+1} f$ et $f$ coïncident sur $\mathbb{Z} / 2^{N+1}$, donc sur $\mathbb{Z} / 2^{N}$. 
iii) est évident

$$
\left(I_{N+1}-I_{N}\right) f=\sum_{k \in \mathbb{Z}}\left(f\left(\frac{k}{2^{N+1}}\right)-I_{N} f\left(\frac{k}{2^{N+1}}\right)\right) \Phi_{N+1}\left(2^{N+1} x-k\right) .
$$

Nous arrivons au point iv). Remarquons d'abord que l'équivalence entre K2) et K3) est immédiate: il suffit de vérifier qu'il existe une constante $C_{0}$ telle que pour tout $N$ on ait

$$
\frac{1}{C_{0}}\left(\sum_{k \in \mathbb{Z}}\left|\lambda_{k}\right|^{p}\right)^{1 / p} \leq\left\|\sum_{k \in \mathbb{Z}} \lambda_{k} \Phi_{N}(x-k)\right\|_{p} \leq C_{0}\left(\sum_{k \in \mathbb{Z}}\left|\lambda_{k}\right|^{p}\right)^{1 / p}
$$

Pour cela on note $\Omega_{N}$ la fonction duale de $\Phi_{N}$

$$
\hat{\Omega}_{N}(\xi)=\frac{\hat{\Phi}_{N}(\xi)}{\sum_{k \in \mathbb{Z}}\left|\hat{\Phi}_{N}(\xi+2 k \pi)\right|^{2}} .
$$

Il est immédiat que $\Omega_{N} \in \mathcal{S}$ et que $\Omega_{N} \rightarrow \omega_{\infty}$ dans $\mathcal{S}$ quand $N \rightarrow+\infty$, où

$$
\hat{\omega}_{\infty}=\frac{\hat{\varphi}_{\infty}}{\sum_{k \in \mathbb{Z}}\left|\hat{\varphi}_{\infty}(\xi+2 k \pi)\right|^{2}} .
$$

On a alors

$$
\begin{gathered}
\left\|\sum_{k \in \mathbb{Z}} \lambda_{k} \Phi_{N}(x-k)\right\|_{p} \leq\left(\sum_{k \in \mathbb{Z}}\left|\lambda_{k}\right|^{p}\right)^{1 / p}\left\|\sum_{k \in \mathbb{Z}}\left|\Phi_{N}(x-k)\right|\right\|_{\infty}, \\
\left(\sum_{k \in \mathbb{Z}}\left|\lambda_{k}\right|^{p}\right)^{1 / p}=\sup _{\left\|\mu_{k}\right\|_{\ell p^{\prime}} \leq 1}\left|\left\langle\sum_{k} \lambda_{k} \Phi_{N}(x-k), \sum_{k} \mu_{k} \Omega_{N}(x-k)\right\rangle\right| \\
\leq\left\|\sum_{k} \lambda_{k} \Phi_{N}(x-k)\right\|_{p}\left\|\sum_{k \in \mathbb{Z}}\left|\Omega_{N}(x-k)\right|\right\|_{\infty} .
\end{gathered}
$$

(42) est alors immédiat.

K3) implique K1) est assez facile et plusieurs arguments peuvent s'appliquer. Par exemple, on prend une fonction d'échelle orthogonale phi de Lemarié-Meyer et psi son ondelette associée. On sait [19] que l'on a

$$
\begin{aligned}
\| \sum_{k \in \mathbb{Z}} \lambda_{k} \operatorname{phi}(x-k) & +\sum_{N=0}^{\infty} \sum_{k \in \mathbb{Z}} \lambda_{N, k} \operatorname{psi}\left(2^{N} x-k\right) \|_{B_{q}^{s, p}} \\
\leq & C_{s, p, q}\left(\left\|\lambda_{k}\right\|_{p}+\left\|2^{N(s-1 / p)}\right\| \lambda_{N, k}\left\|_{\ell^{p}(k)}\right\|_{\ell^{q}(N)}\right),
\end{aligned}
$$


pour tous $s \in \mathbb{R}$ et $p, q \in[1,+\infty]$; par ailleurs l'opérateur $T$ défini sur $\mathcal{S}$ par

$$
\begin{cases}T(\operatorname{phi}(x-k))=\Phi_{0}(x-k), & k \in \mathbb{Z}, \\ T\left(\operatorname{psi}\left(2^{N} x-k\right)\right)=\Psi_{N}\left(2^{N} x-k\right), & N \geq 0, k \in \mathbb{Z},\end{cases}
$$

est un opérateur pseudo-différentiel exotique appartenant à la classe $S_{1}^{0,1}$ : son symbole est donné par

$$
\begin{aligned}
\sigma(x, \xi)= & \widehat{\operatorname{phi}}(\xi) \sum_{k \in \mathbb{Z}} \hat{\Phi}_{0}(\xi+2 k \pi) e^{i k x} \\
& +\sum_{N=0}^{\infty} \widehat{\operatorname{psi}}\left(\frac{\xi}{2^{N}}\right) \sum_{k \in \mathbb{Z}} \hat{\Psi}_{N}\left(\frac{\xi}{2^{N}}+2 k \pi\right) e^{i 2^{N} k x}
\end{aligned}
$$

Or la classe $S_{1}^{0,1}$ opère continûment sur $B_{q}^{s, p}$ pour tout $s>0$ [28] et donc K3) implique K1) est prouvé.

Il reste à prouver K1) implique K3). Par dualité, cela revient à vérifier que (en notant $p^{\prime}=p /(p-1)$ et $q^{\prime}=q /(q-1)$ )

$$
\begin{aligned}
& \left\|\sum_{\lambda_{k} \in \mathbb{Z}} \lambda_{k} \delta(x-k)+\sum_{N=0}^{\infty} \sum_{k \in \mathbb{Z}} \lambda_{N, k} \theta_{N}\left(2^{N} x-k\right)\right\|_{B_{q^{\prime}}^{-s, p^{\prime}}} \\
& \quad \leq C_{s, p, q}\left(\left\|\lambda_{k}\right\|_{p^{\prime}}+\left\|2^{N\left(-s-1 / p^{\prime}\right)}\right\| \lambda_{N, k}\left\|_{\ell^{p^{\prime}}(k)}\right\|_{\ell^{q^{\prime}}(N)}\right) .
\end{aligned}
$$

Pour cela, on va vérifier que si $\sigma<-1 / p$ alors il existe $C_{\sigma, p, q}>0$ tel que pour tout $N>-\sigma$ et toute suite $\left\{\lambda_{N, k}\right\}$ on ait

$$
\left\|\sum_{k \in \mathbb{Z}} \lambda_{N, k} \theta_{N}\left(2^{N} x-k\right)\right\|_{B_{q^{\prime}}^{\sigma, p^{\prime}}} \leq C_{\sigma, p, q}\left\|\lambda_{N, k}\right\|_{\ell^{p^{\prime}}} 2^{N\left(-1 / p^{\prime}+\sigma\right)} .
$$

Supposons (44) établi et choisissons $\alpha>0$ tel que $-s+\alpha<-1 / p$ et fixons $N_{0}>s+\alpha$. On a alors

- pour $N<N_{0}$

$$
\left\|\sum_{k \in \mathbb{Z}} \lambda_{N, k} \theta_{N}\left(2^{N} x-k\right)\right\|_{B_{q^{\prime}}^{s, p^{\prime}}} \leq C_{N}\left\|\lambda_{N, k}\right\|_{\ell^{p^{\prime}}(k)},
$$

il suffit d'écrire $\|F\|_{B_{q^{\prime}}^{-s, p^{\prime}}} \leq\|F\|_{B_{p^{\prime}}^{-s+\alpha, p^{\prime}}}$ et de remarquer que la norme de $B_{p}^{s-\alpha, p}$ est localisable [22] de sorte que

$$
\left(\sum_{k \in \mathbb{Z}}|f(k)|^{p}\right)^{1 / p} \leq C_{s-\alpha, p}\|f\|_{B_{p}^{s, p}} .
$$


- pour $N \geq N_{0}$ : on a pour $\eta= \pm \alpha$

$$
\begin{aligned}
\left\|\sum_{k \in \mathbb{Z}} \lambda_{N, k} \theta_{N}\left(2^{N} x-k\right)\right\|_{B_{q^{\prime}}^{-s+2, p^{\prime}}} & \leq C\left\|\lambda_{N, k}\right\|_{\ell^{p^{\prime}}} 2^{N\left(-1 / p^{\prime}-s+\eta\right)} \\
& =C 2^{N \eta} \varepsilon_{N}, \quad \text { où } \varepsilon_{N} \in \ell^{q} .
\end{aligned}
$$

Si

$$
\omega=\sum_{N \geq N_{0}} \sum_{k \in \mathbb{Z}} \lambda_{N, k} \theta_{N}\left(2^{N} x-k\right)=\sum_{N \geq N_{0}} \omega_{N}
$$

la décomposition de Littlewood-Paley $\omega=S_{0} \omega+\sum_{j \geq 0} \Delta_{j} \omega$ permet facilement de montrer que $\omega \in B_{q^{\prime}}^{-s, p^{\prime}}$

$$
\left\|S_{0} \omega\right\|_{p^{\prime}} \leq \sum_{N \geq N_{0}}\left\|S_{0} \omega_{N}\right\|_{p^{\prime}} \leq C \sum_{N \geq N_{0}} 2^{-N \alpha} \varepsilon_{N}<+\infty,
$$

tandis que

$$
\begin{aligned}
\left\|\Delta_{j} \omega\right\|_{p^{\prime}} \leq & \sum_{N \geq N_{0}}\left\|\Delta_{j} \omega_{N}\right\|_{p^{\prime}} \\
\leq & \sum_{N \geq N_{0}, N \leq j}\left\|\omega_{N}\right\|_{B_{q^{\prime}}^{-s+\alpha, p^{\prime}}} 2^{j(s-\alpha)} \\
& \quad+\sum_{N \geq N_{0}, N \geq j}\left\|\omega_{N}\right\|_{B_{q^{\prime}}^{-s-\alpha, p^{\prime}}} 2^{j(s+\alpha)}
\end{aligned}
$$

de sorte que

$$
2^{-j s}\left\|\Delta_{j} \omega\right\|_{p^{\prime}} \leq C \sum_{N} 2^{-\alpha|j-N|} \varepsilon_{N}
$$

et donc $\left\{2^{-j s}\left\|\Delta_{j} \omega\right\|_{p^{\prime}}\right\} \in \ell^{q}(j)$ si $\left\{\varepsilon_{N}\right\} \in \ell^{q}(N)$.

Il reste à vérifier (44). C'est presque immédiat. En effet, si $\Omega$ est à décroissance rapide dans $B_{q^{\prime}}^{\sigma+\varepsilon, p^{\prime}}$, on a

$$
\left\|\sum_{k} \lambda_{k} \Omega(x-k)\right\|_{B_{q^{\prime}}^{\sigma, p^{\prime}}} \leq\left(\sum_{k}\left|\lambda_{k}\right|^{p^{\prime}}\right)^{1 / p^{\prime}} C(\Omega)
$$

(ce quelque soit $\sigma \in \mathbb{R}$ ). En effet, c'est évident si $p^{\prime}=q^{\prime}$ par localisation; si $p^{\prime} \neq q^{\prime}$ on écrit $B_{q^{\prime}}^{\sigma, p^{\prime}} \subset B_{p^{\prime}}^{\sigma+\varepsilon / 2, p^{\prime}} \subset B_{q^{\prime}}^{\sigma+\varepsilon, p^{\prime}}$. Si $\sigma>0$, on en conclut que pour $A \geq 1$

$$
\left\|\sum_{k} \lambda_{k} \Omega(A x-k)\right\|_{B_{q^{\prime}}^{\sigma, p^{\prime}}} \leq C(\Omega)\left(\sum_{k}\left|\lambda_{k}\right|^{p^{\prime}}\right)^{1 / p^{\prime}} A^{-1 / p^{\prime}+\sigma} .
$$


Si $\sigma<0$, cela reste vrai pourvu que $\int x^{k} \Omega d x=0$ pour $0 \leq k \leq[-\sigma]=$ $N_{0}$ : dans ce cas on sait que $\Omega=(d / d x)^{N_{0}+1} \tilde{\Omega}$ où $\tilde{\Omega}$ est à décroissance rapide dans $B_{q^{\prime}}^{\sigma+\varepsilon+N_{0}+1, p^{\prime}}$. (44) est donc immédiat, car les $\theta_{N}$ vérifient que pour tout $p \in \mathbb{N},\left\{x^{p} \theta_{N}\right\}$ est une famille bornée dans $B_{q^{\prime}}^{\sigma+\varepsilon, p^{\prime}}$ si $\sigma+\varepsilon<-1 / p$ et de plus $\left\langle\theta_{N}, x^{p}\right\rangle=0$ pour $0 \leq p \leq N$.

Le Théorème 4 est donc démontré.

Remarques. i) Si $0<s \leq 1 / p$ et si

$$
\left\|2^{N(s-1 / p)}\right\| \lambda_{N, k}\left\|_{\ell^{p}(k)}\right\|_{\ell^{q}(N)}<+\infty,
$$

on a démontré que

$$
\sum_{N=0}^{\infty} \sum_{k \in \mathbb{Z}} \lambda_{N, k} \Psi_{N}\left(2^{N} x-k\right)
$$

définissait un élément $f$ de $B_{q}^{s, p}$. Cependant on ne peut écrire $\lambda_{N, k}=$ $2^{N}\left\langle f, \theta_{N}\left(2^{N} x-k\right)\right\rangle$ puisque $\theta_{N} \notin\left(B_{q}^{s, p}\right)^{*}$. En particulier, on peut avoir une série d'ondelettes convergeant vers 0 dans $B_{q}^{s, p}$ avec des coefficients $\lambda_{N, k}$ non nuls.

ii) Le problème de l'interpolation des fonctions périodiques décrit à la fin de la section $\mathrm{V}$ est évident ici: si $f \in B_{\infty}^{r, \infty}(\mathbb{R} / 2 \pi \mathbb{Z})$ on a $\left\|f-I_{N} f\right\|_{\infty} \leq C /\left(2^{N}\right)^{r}$, ce qui est mieux que pour le système de Rvachev ou pour le système trigonométrique.

\section{Le problème de la phase.}

Dans la section précédente, nous avons décrit les fonctions d'échelle interpolantes et leur approximation par des fonctions d'échelle interpolantes (stationnaires ou non-stationnaires) à support compact.

Par intégration par parties [17], nous pouvons de même approximer des fonctions d'échelle en bi-orthogonalité avec des fonctions splines: en effet dire que la fonction $\varphi$ est une fonction d'échelle régulière en dualité avec le $B$-spline $N$ de degré $k\left(\hat{N}=\left(\left(1-e^{-i \xi}\right) / i \xi\right)^{k+1}\right)$ revient à dire que la fonction $\Phi$, définie $\operatorname{par}(d / d x)^{k+1} \Phi=\Delta^{k+1} \varphi$ où $\Delta \varphi=$ $\varphi(x+1)-\varphi(x)$, est une fonction d'échelle interpolante.

Il serait de même utile de savoir approximer les fonctions d'échelle orthogonales par des fonctions d'échelle orthogonales à support compact. Nous pourrions alors construire une base de Berkolaïko-Novikov 
sur le modèle de l'interpolante de Kharkov, et obtenir des fonctions d'échelle orthogonales non-stationnaires à support compact qui convergeraient dans $\mathcal{S}$, de sorte que la base d'ondelettes non stationnaires serait une "base universelle" (au moins dans les échelles $B_{q}^{s, p}, F_{q}^{s, p}, \ldots$ ) à l'instar des bases de Lemarié-Meyer.

Nous butons alors sur un problème: nous n'avons pas de description directe des filtres d'échelle orthogonaux à réponse impulsionnelle finie. C'est-à-dire que pour construire un tel filtre $m_{0}$, on construit d'abord $\left|m_{0}(\xi)\right|^{2}$ (qui est un filtre d'échelle interpolant) puis on en prend une "racine carrée polynômiale" grâce au lemme de Riesz: on a alors $m_{0}(\xi)=\left|m_{0}(\xi)\right| e^{-i \omega(\xi)}$ où la phase $\omega$ dépend du choix des racines qu'on a conservées pour définir $m_{0}$. Comment alors contrôler cette phase pour que la convergence de $\left|m_{0}\right|^{2}$ entraine celle de $m_{0}$ ?

Ce problème reste extrêmement délicat à traiter. Il a motivé (à côté d'autres raisons) l'étude d'un cas particulier: les filtres de Daubechies $m_{N}(\xi)$. Pour lesquels on a

$$
\lim _{N \rightarrow+\infty}\left|m_{N}(\xi)\right|^{2}= \begin{cases}1, & \text { si }|\xi|<\frac{\pi}{2} \\ 0, & \text { si } \frac{\pi}{2}<|\xi| \leq \pi, \xi= \pm \frac{\pi}{2} .\end{cases}
$$

Dans ce cas, une étude de la phase peut être poussée assez loin [16], essentiellement parce que $\left|m_{N}(\xi)\right|^{2}$ est donné par

$$
\left|m_{N}(\xi)\right|^{2}=P_{2 N+1}\left(\frac{1+\cos \xi}{2}\right),
$$

où $P_{2 N+1}$ est le polynôme de Bernstein associé à la fonction analytique par morceaux $\chi_{[1 / 2,1]}$. Mais nous ne disposons pas encore de résultats applicables à une interpolante de Lemarié-Meyer.

Pour avoir une "base universelle", on peut, au lieu de chercher à tout prix une base orthogonale, scinder $\left|m_{0}(\xi)\right|^{2}$ en un produit $m_{1}(\xi)$ $\overline{m_{2}(\xi)}$ en imposant le convergence dans $C^{\infty}$ de $m_{1}$ et de $m_{2}$. On obtiendrait alors une base bi-orthogonale d'ondelettes non-stationnaires qui serait une "base universelle" et dont les fonctions d'échelle nonstationnaires tendraient vers des fonctions d'échelle bi-orthogonales stationnaires. 


\section{Filtres peu réguliers.}

Comme nous l'avons expliqué ci-dessus, la possibilité de décrire le comportement asymptotique de la phase des filtres de Daubechies tient à la simplicité de la limite de $\left|m_{N}(\xi)\right|^{2}$, c'est-à-dire de la fonction qui définit en retour les filtres de Daubechies comme des polynômes de Bernstein. Le point principal est que

$$
\frac{d}{d x} \chi_{[1 / 2,+\infty[}=\delta\left(x-\frac{1}{2}\right),
$$

ce qui donne pour le polynôme

$$
\sum_{k>N} C_{2 N+1}^{k} x^{k}(1-x)^{2 N+1-k}=P_{2 N+1}(x)
$$

la formule

$$
\frac{d}{d x} P_{2 N+1}(x)=(N+1) C_{2 N+1}^{N+1} x^{N}(1-x)^{N},
$$

de sorte que $P_{2 N+1}$ s'écrit simplement

$$
P_{2 N+1}(x)=\int_{0}^{x} \frac{(2 N+1) !}{(N !)^{2}}(t(1-t))^{N} d t .
$$

Par ailleurs, les fonctions d'échelle $\varphi_{N}$ héritent de la mauvaise localisation de fonction d'échelle $\varphi_{\infty}$ associée à $m_{0}=\chi_{[-\pi / 2, \pi / 2]}\left(\varphi_{\infty}=\right.$ $(\sin \pi x) /(\pi x))$ de sorte que

$$
\limsup _{N \rightarrow+\infty} \inf _{x_{0}} \int\left|x-x_{0}\right|^{2}\left|\varphi_{N}(x)\right|^{2} d x=+\infty .
$$

On peut alors chercher à adoucir la limite de $m_{N}$ tout en conservant des propriétés remarquables pour cette limite, afin d'être en mesure de contrôler la phase. Une idée prometteuse est alors de prendre pour $\left|m_{\infty}(\xi)\right|^{2}$ un spline par morceaux, c'est-à-dire un exemple élémentaire de fonction analytique par morceaux dont les dérivées se calculent aisément; un tel choix offre par ailleurs l'avantage de permettre des calculs explicites sur les filtres interpolants construits à l'aide de polynômes de Bernstein, donc d'échantillonnages de $\left|m_{\infty}(\xi)\right|^{2}$. Cependant, la convergence des filtres ne peut plus avoir lieu dans $C^{\infty}$ et la convergence des fonctions d'échelle ne peut plus être rapide. Il faut alors reprendre toute 
la théorie ci-dessus développée pour l'adapter aux filtres peu réguliers et aux fonctions d'échelle peu décroissantes... A titre d'exemple, nous signalons que le lecteur intéressé trouvera dans [15] une démonstration du résultat suivant (qui généralise la Proposition 1).

Proposition 9. Soit $m_{0} \in H^{1 / 2+\varepsilon}(\mathbb{R} / 2 \pi \mathbb{Z})$ où $\varepsilon>0$ telle que $m_{0}(0)=$ $1, m_{0}(\xi)=\overline{m_{0}(-\xi)}$. On note $\hat{\varphi}$ la fonction

$$
\hat{\varphi}(\xi)=\prod_{j=1}^{\infty} m_{0}\left(\frac{\xi}{2^{j}}\right)
$$

et $T_{2}$ l'opérateur agissant sur les fonctions $2 \pi$-périodiques défini par

$$
T_{2} f=\left|m_{0}\left(\frac{\xi}{2}\right)\right|^{2} f\left(\frac{\xi}{2}\right)+\left|m_{0}\left(\frac{\xi}{2}+\pi\right)\right|^{2} f\left(\frac{\xi}{2}+\pi\right) .
$$

Alors $m_{0}$ satisfait le critère d'Albert Cohen et $\sup _{N}\left\|T_{2}^{N}(1)\right\|_{\infty}<+\infty$ si et seulement si $(1+|x|)^{1 / 2+\varepsilon} \varphi \in L^{2}(\mathbb{R})$ et la famille $\{\varphi(x-k)\}_{k \in \mathbb{Z}}$ est une base de Riesz d'un sous-espace fermé de $L^{2}(\mathbb{R})$. De plus pour tout $\alpha>1 / 2,|x|^{\alpha} \varphi \in L^{2}(\mathbb{R})$ si et seulement si $m_{0} \in H^{\alpha}(\mathbb{R} / 2 \pi \mathbb{Z})$.

Remerciements. Je tiens à remercier les nombreuses personnes sans qui ce travail n'aurait pu même être entamé: Djalil Kateb (de l'Université de Compiègne) pour nos travaux communs sur le problème de la phase des filtres d'échelle et nos longues discussions sur la fonction de Rvachev, Yves Meyer (de l'Université de Paris-Dauphine) pour avoir attiré mon attention sur la très belle thèse de Loïc Hervé, Igor Novikov (de l'Université de Voronezh) pour l'envoi de ses manuscrits sur la base de Berkolaïko et Novikov, Amos Ron (de l'Université de WisconsinMadison) pour l'envoi de nombreux tirés à part sur les analyses multirésolution non stationnaires, Winifried Sickel (de l'Université de Iena) pour ses précieuses indications sur la théorie de l'approximation et la fonction de Rvachev, ainsi que Josette Dumas qui a tapé le manuscrit du présent texte.

\section{References.}

[1] Akansu, A. N., Caglar, H., A generalized parametric PR-QMF design technique based on Bernstein polynomial approximation. IEEE Trans. Signal Processing 41 (1993), 2314-2321. 
[2] Berkolaiko, M., Novikov, I., On infinitely smooth almost wavelets with compact support. Dokl. Rus. Acad. Nauk 326 (1992), 935-938. (In russian).

[3] Bernstein, N., Démonstration du théorème de Weierstrass, fondée sur le calcul des probabilités. C. R. Soc. Math. Kharkov 13 (1913), 1-2.

[4] Cohen, A., Ondelettes, analyses multi-résolutions et traitement numérique du signal. Thèse, Univ. Paris IX, 1990.

[5] Cohen, A., Conze, J. P., Régularité des bases d'ondelettes et mesures ergodiques. Revista Mat. Iberoamericana 8 (1992), 351-366.

[6] Cohen, A., Daubechies, I., A stability criterion for bi-orthogonal wavelets and their related subband coding scheme. Duke Math. J. 68 (1992), 313-335.

[7] Cohen, A., Dyn, N., Non-stationary subdivision schemes and multiresolution analysis. SIAM J. Math. Anal. 27 (1996), 1745-1769.

[8] Daubechies, I., Orthogonal bases of compactly supported wavelets. Comm. Pure and Applied Math. 41 (1988), 909-996.

[9] Daubechies. I., Lagarias, J., Two-scale difference equation. I: Existence and global regularity of solutions. SIAM J. Math. Anal. 22 (1991), 1388-1410.

[10] De Boor, C., De Vore, R., Ron, A., The structure of finitely generated shift-invariant spaces in $L^{2}\left(\mathbb{R}^{d}\right)$. J. Funct. Anal. 119 (1994), 211-232.

[11] Dyn, N., Ron, A., Multiresolution analysis by infinitely differentiable compactly supported functions. Applied and Comp. Harmonic Anal. 2 (1995), 15-20.

[12] Eirola, T., Sobolev characterization of solutions of dilation equations. SIAM J. Math. Anal. 23 (1992), 1015-1030.

[13] Hermann, O., On the approximation problem in non-recursive digital filter design. IEEE Trans. on Circuit Theory (1971), 411-413.

[14] Hervé L., Méthodes d'opérateurs quasi-compacts en analyse multi-résolution, applications à la construction de bases d'ondelettes et à l'interpolation. Thèse, Univ. Rennes I, 1992.

[15] Kahane, J.-P., Lemarié-Rieusset, P. G., Fourier series and wavelets. Gordon and Breach, 1995.

[16] Kateb, D., Lemarié-Rieusset, P. G., On the phase of the Daubechies filters. To appear in Revista Mat. Iberoamericana

[17] Lemarié-Rieusset, P. G., Analyses multi-résolutions non orthogonales, commutation entre projecteurs et dérivation et ondelettes vecteurs à divergence nulle. Revista Mat. Iberoamericana 8 (1992), 221-237.

[18] Lemarié-Rieusset, P. G., Polynômes de Bernstein en théorie des ondelettes. C. R. Acad. Sci. Paris 319 (1994), 21-24. 
[19] Lemarié-Rieusset, P. G., Meyer, Y., Ondelettes et bases hilbertiennes. Revista Mat. Iberoamericana 2 (1986), 1-18.

[20] Mallat, S., Multiresolution approximation and wavelets. Trans. Amer. Math. Soc. 315 (1989), 69-88.

[21] Meyer, Y., Ondelettes et opérateurs. I: Ondelettes. Herman, 1990.

[22] Peetre, J., New thoughts on Besov spaces.

[23] Rioul, O., Ondelettes régulières, application à la compression d'images fixes. Thèse, CNET, 1993.

[24] Ron, A., A necessary and sufficient condition for the linear independence of the interger translate of a compactly supported distribution. Constructive Approx. 5 (1989), 297-308.

[25] Rudin, W., Real and complex analysis. McGraw Hill, 1974.

[26] Rvachev, V. A., Compactly supported solutions of functional differential equations and their applications. Russian Math. Surveys 45 (1990), 87120.

[27] Rvachev, V. A., Rvachev, V. L., Neklasscheskie metody teorii priblizherii $v$ kraevykh zadachakh. Naukova Dumka, 1979.

[28] Stein, E. M., Harmonic analysis: real-variable methods, orthogonality and oscillatory integrals. Princeton Univ. Press, 1993.

[29] Strichartz, R., How to make wavelets. Amer. Math. Monthly 100 (1993), 539-556.

[30] Villemoes, L., Wavelet analysis of two-scale difference equations. SIAM J. Math. Anal. 5 (1994), 1433-1460.

[31] Zygmund, A., Trigonometric series. Vol. I, II. Cambridge Univ. Press, 1959.

Recibido: 2 de octubre de 1.995

Revisado: 26 de abril de 1.996

Pierre Gilles Lemarié-Rieusset Université d'Evry

Mathématiques-Bd des Coquibus 91025 Evry Cedex, FRANCE lemarie@lami.univ-evry.fr 